Supporting Inforamtion

\title{
Synthesis of Benzoxaboroles by ortho-Oxalkylation of Arylboronic Acids with Aldehydes/Ketones in the Presence of Brønsted Acids
}

\author{
Jing Zhao, Jiuxi Chen, Qing Xu, Huan $\mathrm{Li}^{*}$ \\ College of Chemistry and Materials Engineering, Wenzhou University, Wenzhou, Zhejiang, \\ 325035, China \\ Email: lihuan@wzu.edu.cn
}

\section{Contents}

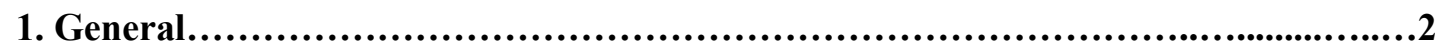

2. Experimental procedure and spectra data.......................................2

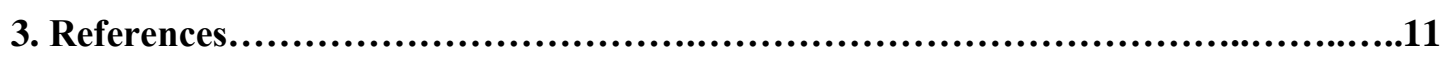

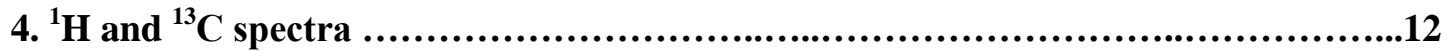




\section{General Information}

All reactions were performed in $\mathrm{N}_{2}$ atmosphere. All solvents were purchased from commercial source and used without further purification: chloroform $\left(\mathrm{CHCl}_{3}\right)$ (AR grade, Zhongxing Chem); carbon tetrachloride $\left(\mathrm{CCl}_{4}\right)$ ( $\mathrm{AR}$ grade, Jiani Chem); n-hexane (AR grade, Sinopharm Chem); dichloromethane (DCM), 1,2-dichloroethane (DCE), acetonitrile $\left(\mathrm{CH}_{3} \mathrm{CN}\right)$ and tetrahydrofuran (THF) (AR grade, extra dry, with molecular sieves, Energy Chemical). The eluting solvents for chromatography, including petroleum ether (PE), ethyl acetate (EA) and dichloromethane (DCM), were purchased from Greagent (AR grade). The reagents were obtained from commercial suppliers, and used without further purification. For chromatography, 200-300 mesh silica gel (Qingdao, China) was employed. ${ }^{1} \mathrm{H}$ NMR and ${ }^{13} \mathrm{C}$ NMR spectra were recorded on WNMR-1-400MHz (Zhongke-Niujin) and AVANCE III AV500 MHz (Brucker) spectrometer. The ${ }^{1} \mathrm{H}$ NMR chemical shifts converted to the TMS scale $\left(\delta_{\mathrm{TMS}}=0.00 \mathrm{ppm}\right)$, while the ${ }^{13} \mathrm{C}$ NMR chemical shifts converted to the corresponding solvent scale (DMSO- $d 6, \quad \delta_{\mathrm{C}}=39.70 \mathrm{ppm}$ ). HRMS was measured on Waters Xero UPLC Q-TOF instrument (ESI). The melting point was measured on WRS-1B (Shanghai Shenguang).

\section{Experimental procedures}

General procedure: To a mixture of arylboronic acid, aldehyde or ketone in $\mathrm{CHCl}_{3}$ was added $\mathrm{Br} ø$ nsted acid. The reaction mixture was stirred at room temperature (rt) or noted temperature in dry block heater, and the reaction was monitored by TLC analysis. Once the arylboronic acid was totally converted, or the amount of product did not increase anymore, the solvent was removed and the residue was purified by silica gel chromatography.

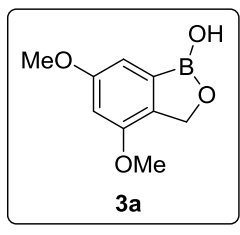

\section{4,6-dimethoxybenzo[c] [1,2] oxaborol-1(3H)-ol (3a)}

Conditions: 3,5-dimethoxyphenylboronic acid (182 mg, $1 \mathrm{mmol}$ ), paraformaldehyde (36 mg, 1.2 mmol), TFA ( $23 \mathrm{mg}, 15 \mu \mathrm{L}, 0.2$ equiv.), $\mathrm{CHCl}_{3}(10 \mathrm{~mL}), \mathrm{rt}, 48 \mathrm{~h}$. The title compound was obtained as white solid (155 mg, 80\%). m.p. $139.7-140.4{ }^{\circ} \mathrm{C} . R_{\mathrm{f}}=0.38$ (PE/EA, $\left.5 / 1\right)$.

${ }^{1} \mathrm{H}$ NMR (400 MHz, DMSO- $\left.d 6\right): \delta 9.16(\mathrm{~s}, 1 \mathrm{H}), 6.83(\mathrm{~d}, J=1.7 \mathrm{~Hz}, 1 \mathrm{H}), 6.63(\mathrm{~d}, J=1.8 \mathrm{~Hz}, 1 \mathrm{H})$, $4.85(\mathrm{~s}, 2 \mathrm{H}), 3.79(\mathrm{~s}, 3 \mathrm{H}), 3.78(\mathrm{~s}, 3 \mathrm{H})$.

${ }^{13} \mathrm{C}$ NMR (125 MHz, DMSO-d6): $\delta$ 160.9, 154.5, 134.1, 104.9, 101.3, 67.9, 55.5, 55.2.

HRMS (ESI, $m / z$ ) calcd for $\mathrm{C}_{9} \mathrm{H}_{12} \mathrm{BO}_{4}[\mathrm{M}+\mathrm{H}]^{+}:$195.0823, found: 195.0822 .

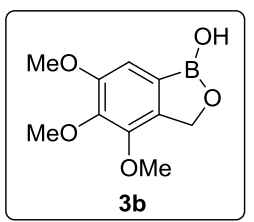

$3 \mathrm{H}), 3.77$ (s, 3H).

\section{4,5,6-trimethoxybenzo[c] $[1,2]$ oxaborol-1(3H)-ol (3b)}

Conditions: 3,4,5-trimethoxyphenylboronic acid (106 mg, $0.5 \mathrm{mmol})$, paraformaldehyde (18 $\mathrm{mg}$, $0.6 \mathrm{mmol})$, TFA ( $171 \mathrm{mg}, 111 \mu \mathrm{L}, 3$ equiv.), $\mathrm{CHCl}_{3}(5 \mathrm{~mL}) \mathrm{rt}, 17 \mathrm{~h}$. The title product was obtained as yellow viscous liquid $(62 \mathrm{mg}, 55 \%) . R_{\mathrm{f}}=0.38(\mathrm{PE} / \mathrm{EA}, 5 / 1)$.

${ }^{1} \mathrm{H}$ NMR (500 MHz, DMSO-d6): $\delta 9.10$ (s, 1H), 7.08 (s, 1H), 4.97 (s, 2H), 3.84 (s, 3H), 3.81 (s,

${ }^{13}$ C NMR (125 MHz, DMSO-d6): $\delta$ 153.7, 147.2, 143.4, 138.1, 108.4, 67.9, 60.5, 56.0, 56.1.

HRMS (ESI, $m / z$ ) calcd for $\mathrm{C}_{10} \mathrm{H}_{14} \mathrm{BO}_{5}[\mathrm{M}+\mathrm{H}]^{+}: 225.0929$, found: 225.0925 .

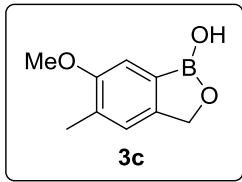

\section{6-methoxy-5-methylbenzo[c] [1,2] oxaborol-1(3H)-ol (3c)}

Conditions: 3-methoxy-4-methylphenylboronic acid (166 mg, $1 \mathrm{mmol}$ ), paraformaldehyde (36 mg, $1.2 \mathrm{mmol}$ ), TFA (456 mg, $297 \mu \mathrm{L}, 4$ equiv.), $\mathrm{CHCl}_{3}(10 \mathrm{~mL}), \mathrm{rt}, 22 \mathrm{~h}$. The title product was obtained as pink solid (62 mg, 35\%). m.p. $135.1-136.3^{\circ} \mathrm{C} . R_{\mathrm{f}}=0.40(\mathrm{PE} / \mathrm{EA}, 5 / 1)$.

${ }^{1} \mathrm{H}$ NMR (400 MHz, DMSO-d6): $\delta$ 9.01(s, 1H), 7.22 (s, 1H), 7.17 (s, 1H), 4.88 (s, 2H), $3.81(\mathrm{~s}, 3 \mathrm{H}), 2.20$ (s, 3H).

${ }^{13} \mathrm{C}$ NMR (125 MHz, DMSO-d6): $\delta$ 157.0, 146.2, 129.6, 123.2, 110.8, 69.7, 55.3, 16.8.

HRMS (ESI, $m / z$ ) calcd for $\mathrm{C}_{9} \mathrm{H}_{12} \mathrm{BO}_{3}[\mathrm{M}+\mathrm{H}]^{+}: 179.0874$, found: 179.0862 . 


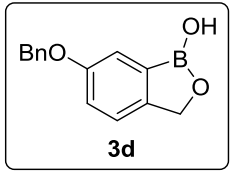

6-(benzyloxy) benzo[c] [1,2] oxaborol-1(3H)-ol (3d)

Conditions: 3-benzyloxybenzeneboronic acid (114 mg, $0.5 \mathrm{mmol})$, paraformaldehyde (18 mg, 0.6 mmol), TFA (285 mg, $186 \mu \mathrm{L}, 5$ equiv.), $\mathrm{CHCl}_{3}(5 \mathrm{~mL}), \mathrm{rt}, 24 \mathrm{~h}$. The title product was obtained as white solid (58 mg, 48\%). The spectra data matched the literature. ${ }^{1} R_{\mathrm{f}}=0.38(\mathrm{PE} / \mathrm{EA}, 5 / 1)$.

${ }^{1} \mathrm{H}$ NMR (400 MHz, DMSO-d6): $\delta 9.15$ (s, 1H), 7.47-7.45 (m, 2H), 7.41-7.38 (m, 2H), 7.35-7.31 (m, 3H), $7.13(\mathrm{dd}, J=$ 8.2, 1.9 Hz, 1H), $5.12(\mathrm{~s}, 2 \mathrm{H}), 4.92(\mathrm{~s}, 2 \mathrm{H})$.

${ }^{13} \mathrm{C}$ NMR (125 MHz, DMSO-d6): $\delta$ 157.8, 146.3, 137.4, 128.5, 127.9, 127.6, 122.5, 118.9, 115.1, 69.7, 69.5.

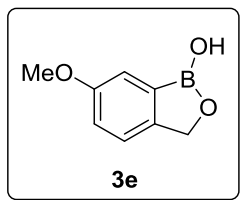

\section{6-methoxybenzo[c] $[1,2]$ oxaborol-1(3H)-ol (3e)}

Conditions: 3-methoxyphenylboronic acid (76 mg, $0.5 \mathrm{mmol})$, paraformaldehyde (18 mg, 0.6 mmol), $p$-toluenesulfonic acid monohydrate $\left(\mathrm{TsOH} \cdot \mathrm{H}_{2} \mathrm{O}\right)\left(48 \mathrm{mg}, 0.25 \mathrm{mmol}, 0.5\right.$ equiv), $\mathrm{CHCl}_{3}$ $(5 \mathrm{~mL}), \mathrm{rt}, 18 \mathrm{~h}$. The title product was obtained as pink solid (32 $\mathrm{mg}, 39 \%)$. The spectra data matched the literature. ${ }^{2} R_{\mathrm{f}}=0.4(\mathrm{PE} / \mathrm{EA}, 5 / 1)$.

${ }^{1} \mathrm{H}$ NMR (400 MHz, DMSO-d6): $\delta 9.13(\mathrm{~s}, 1 \mathrm{H}), 7.31(\mathrm{~d}, J=8.3 \mathrm{~Hz}, 1 \mathrm{H}), 7.26(\mathrm{~d}, J=2.4 \mathrm{~Hz}, 1 \mathrm{H}), 7.05(\mathrm{dd}, J=8.3,2.4$ $\mathrm{Hz}, 1 \mathrm{H}), 4.92(\mathrm{~s}, 2 \mathrm{H}), 3.77$ (s, 3H).

${ }^{13} \mathrm{C}$ NMR (125 MHz, DMSO-d6): $\delta$ 158.7, 146.1, 122.4, 118.1, 113.9, 69.7, 55.3.

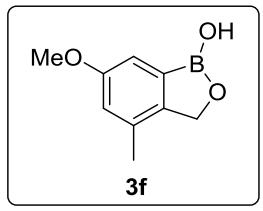

\section{6-methoxy-4-methylbenzo[c] [1,2] oxaborol-1(3H)-ol (3f)}

Conditions: 3-methoxy-5-methylphenylboronic acid ${ }^{3}(83 \mathrm{mg}, 0.5 \mathrm{mmol})$, paraformaldehyde (18 mg, $0.6 \mathrm{mmol})$, TfOH (7.5 mg, $4.4 \mu \mathrm{L}, 0.05$ equiv. $), \mathrm{CHCl}_{3}(5 \mathrm{~mL}), \mathrm{rt}, 8 \mathrm{~h}$. The title product was obtained as white solid (39 mg, 43\%). m.p. $125.4-126{ }^{\circ} \mathrm{C} . R_{\mathrm{f}}=0.30(\mathrm{PE} / \mathrm{EA}, 5 / 1)$.

${ }^{1} \mathrm{H}$ NMR (400 MHz, DMSO-d6): $\delta 9.10(\mathrm{~s}, 1 \mathrm{H}), 7.08(\mathrm{~d}, J=2.1 \mathrm{~Hz}, 1 \mathrm{H}), 6.86(\mathrm{~d}, J=1.8 \mathrm{~Hz}$, 1H), 4.88 (s, 2H), 3.75 (s, 3H), 2.19 (s, 3H).

${ }^{13} \mathrm{C}$ NMR (125 MHz, DMSO-d6): $\delta$ 159.1, 145.1, 132.2, 118.7, 111.2, 69.1, 55.2, 17.5.

HRMS (ESI, $m / z$ ) calcd for $\mathrm{C}_{9} \mathrm{H}_{12} \mathrm{BO}_{3}[\mathrm{M}+\mathrm{H}]^{+}: 179.0874$, found: 179.0871 .

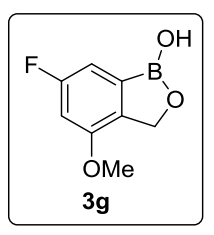

\section{4-fluoro-6-methoxybenzo[c] [1,2] oxaborol-1(3H)-ol (3g)}

Conditions: 3-fluoro-5-methoxybenzeneboronic acid (170 mg, $1 \mathrm{mmol})$, paraformaldehyde (36 mg, $1.2 \mathrm{mmol}), \mathrm{TsOH} \cdot \mathrm{H}_{2} \mathrm{O}\left(95 \mathrm{mg}, 0.5 \mathrm{mmol}, 0.5\right.$ equiv), $\mathrm{CHCl}_{3}(10 \mathrm{~mL})$, rt, $36 \mathrm{~h}$. The title product was obtained as white solid (50 mg, 27\%). m.p. 166.0-168.2 ${ }^{\circ} \mathrm{C} . R_{\mathrm{f}}=0.38(\mathrm{PE} / \mathrm{EA}, 5 / 1)$.

${ }^{1} \mathrm{H}$ NMR (400 MHz, DMSO-d6): $\delta 9.34(\mathrm{~s}, 1 \mathrm{H}), 7.02(\mathrm{dd}, J=7.5,1.4 \mathrm{~Hz}, 1 \mathrm{H}), 6.98(\mathrm{dd}, J=11.5,1.4$ $\mathrm{Hz}, 1 \mathrm{H}), 4.89$ (s, 2H), 3.83 (s, 3H).

${ }^{13} \mathrm{C}$ NMR (125 MHz, DMSO-d6): $\delta 163.2\left(\mathrm{~d}, \mathrm{C}-\mathrm{F},{ }^{1} J \mathrm{C}-\mathrm{F}=241.5 \mathrm{~Hz}\right), 154.8\left(\mathrm{~d}, \mathrm{C}-\mathrm{F},{ }^{3} J \mathrm{C}-\mathrm{F}=9.8 \mathrm{~Hz}\right), 137.3\left(\mathrm{~d}, \mathrm{C}-\mathrm{F},{ }^{4} J\right.$ $\mathrm{C}-\mathrm{F}=0.9 \mathrm{~Hz}), 107.3\left(\mathrm{~d}, \mathrm{C}-\mathrm{F},{ }^{2} J \mathrm{C}-\mathrm{F}=20.3 \mathrm{~Hz}\right), 101.3\left(\mathrm{~d}, \mathrm{C}-\mathrm{F},{ }^{2} J \mathrm{C}-\mathrm{F}=27.7 \mathrm{~Hz}\right), 67.9,55.7$.

HRMS (ESI, $m / z$ ) calcd for $\mathrm{C}_{8} \mathrm{H}_{9} \mathrm{BFO}_{3}[\mathrm{M}+\mathrm{H}]^{+}: 183.0623$, found: 183.0616 .

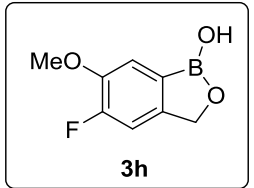

\section{5-fluoro-6-methoxybenzo[c] [1,2] oxaborol-1(3H)-ol (3h)}

Conditions: 4-fluoro-3-methoxyphenylboronic acid ( $85 \mathrm{mg}, 0.5 \mathrm{mmol})$, paraformaldehyde (18 mg, $0.6 \mathrm{mmol})$, triflic acid (TfOH) $(30 \mathrm{mg}, 18 \mu \mathrm{L}, 0.4$ equiv. $), \mathrm{CHCl}_{3}(5 \mathrm{~mL}), 40{ }^{\circ} \mathrm{C}, 72 \mathrm{~h}$. The title product was obtained as white solid (50 mg, 55\%). m.p. $181.2-182.3{ }^{\circ} \mathrm{C} . R_{\mathrm{f}}=0.4(\mathrm{PE} / \mathrm{EA}, 5 / 1)$

${ }^{1} \mathrm{H}$ NMR (400 MHz, DMSO-d6): $\delta 9.19(\mathrm{~s}, 1 \mathrm{H}), 7.44$ (d, $\left.J=8.7 \mathrm{~Hz}, 1 \mathrm{H}\right), 7.28(\mathrm{~d}, J=11.3 \mathrm{~Hz}, 1 \mathrm{H}), 4.91(\mathrm{~s}, 2 \mathrm{H}), 3.86(\mathrm{~s}$, $3 \mathrm{H})$.

${ }^{13} \mathrm{C}$ NMR (125 MHz, DMSO-d6): $\delta 154.1\left(\mathrm{~d}, \mathrm{C}-\mathrm{F},{ }^{1} J \mathrm{C}-\mathrm{F}=248.1 \mathrm{~Hz}\right), 146.8\left(\mathrm{~d}, \mathrm{C}-\mathrm{F},{ }^{3} J \mathrm{C}-\mathrm{F}=3.3 \mathrm{~Hz}\right), 146.9\left(\mathrm{~d}, \mathrm{C}-\mathrm{F},{ }^{2} J\right.$ 
$\mathrm{C}-\mathrm{F}=15.2 \mathrm{~Hz}), 114.5\left(\mathrm{~d}, \mathrm{C}-\mathrm{F},{ }^{3} J \mathrm{C}-\mathrm{F}=2.2 \mathrm{~Hz}\right), 109.3\left(\mathrm{~d}, \mathrm{C}-\mathrm{F},{ }^{2} J \mathrm{C}-\mathrm{F}=19.3 \mathrm{~Hz}\right), 69.5\left(\mathrm{~d}, \mathrm{C}-\mathrm{F},{ }^{4} J \mathrm{C}-\mathrm{F}=2.4 \mathrm{~Hz}\right), 56.0$. HRMS (ESI, $m / z$ ) calcd for $\mathrm{C}_{8} \mathrm{H}_{9} \mathrm{BFO}_{3}[\mathrm{M}+\mathrm{H}]^{+}: 183.0623$, found: 183.0620 .

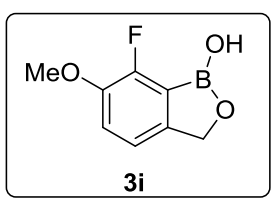

6-methoxybenzo[c] $[1,2]$ oxaborol-1 $(3 \mathrm{H})$-ol $(3 \mathrm{i})$

Conditions: 2-fluoro-3-methoxyphenylboronic acid (170 mg, $1 \mathrm{mmol}$ ), paraformaldehyde (36 $\mathrm{mg}, 1.2 \mathrm{mmol})$, TfOH (30 mg, $18 \mu \mathrm{L}, 0.2$ equiv.), $\mathrm{CHCl}_{3}(10 \mathrm{~mL}), 40{ }^{\circ} \mathrm{C}, 48 \mathrm{~h}$. The title product was obtained as white solid (136 mg, 75\%). m.p. 187.2-190.1 ${ }^{\circ} \mathrm{C} . R_{\mathrm{f}}=0.36$ (PE/EA, 2/1).

${ }^{1} \mathrm{H}$ NMR (500 MHz, DMSO-d6): $\delta 9.28$ (s, 1H), 7.33-7.30 (m, 1H), 7.16 (d, $\left.J=8.1 \mathrm{~Hz}, 1 \mathrm{H}\right), 4.94(\mathrm{~s}, 2 \mathrm{H}), 3.84$ (s, 3H).

${ }^{13} \mathrm{C}$ NMR (125 MHz, DMSO-d6): $\delta 152.4\left(\mathrm{~d}, \mathrm{C}-\mathrm{F},{ }^{1} J \mathrm{C}-\mathrm{F}=249.8 \mathrm{~Hz}\right), 146.6\left(\mathrm{~d}, \mathrm{C}-\mathrm{F},{ }^{3} J \mathrm{C}-\mathrm{F}=7.5 \mathrm{~Hz}\right), 145.8\left(\mathrm{~d}, \mathrm{C}-\mathrm{F},{ }^{2} J\right.$ $\mathrm{C}-\mathrm{F}=11.7 \mathrm{~Hz}), 118.4,117.9\left(\mathrm{~d}, \mathrm{C}-\mathrm{F},{ }^{3} J \mathrm{C}-\mathrm{F}=4.1 \mathrm{~Hz}\right), 69.4,56.7$.

HRMS (ESI, $m / z$ ) calcd for $\mathrm{C}_{8} \mathrm{H}_{9} \mathrm{BFO}_{3}[\mathrm{M}+\mathrm{H}]^{+}: 183.0623$, found: 183.0632 .

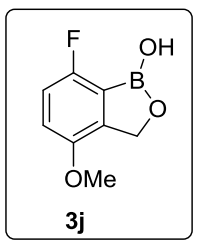

\section{7-fluoro-4-methoxybenzo[c] [1,2] oxaborol-1(3H)-ol (3j)}

Conditions: 2-fluoro-5-methoxyphenylboronic acid (170 mg, $1 \mathrm{mmol})$, paraformaldehyde (36 mg, 1.2 mmol), TfOH (75 mg, $44 \mu \mathrm{L}, 0.4$ equiv. $), \mathrm{CHCl}_{3}\left(10 \mathrm{~mL}, 40{ }^{\circ} \mathrm{C}, 48 \mathrm{~h}\right.$. The title product was obtained as white solid (113 mg, 62\%). m.p. $158.2-159.3{ }^{\circ} \mathrm{C} . R_{\mathrm{f}}=0.37$ (PE/EA, $\left.2 / 1\right)$.

${ }^{1} \mathrm{H}$ NMR (400 MHz, DMSO-d6): $\delta 9.31$ (s, 1H), 7.10-7.01 (m, 2H), 4.92 (s, 2H), 3.80 (s, 3H).

${ }^{13} \mathrm{C}$ NMR (125 MHz, DMSO-d6): $\delta 157.6\left(\mathrm{~d}, \mathrm{C}-\mathrm{F},{ }^{1} J \mathrm{C}-\mathrm{F}=240.0 \mathrm{~Hz}\right), 150.1$ (d, C-F, $\left.{ }^{4} J \mathrm{C}-\mathrm{F}=1.8 \mathrm{~Hz}\right), 143.2\left(\mathrm{~d}, \mathrm{C}-\mathrm{F},{ }^{3} J\right.$ $\mathrm{C}-\mathrm{F}=7.4 \mathrm{~Hz}), 114.6\left(\mathrm{~d}, \mathrm{C}-\mathrm{F},{ }^{3} J \mathrm{C}-\mathrm{F}=7.4 \mathrm{~Hz}\right), 114.3\left(\mathrm{~d}, \mathrm{C}-\mathrm{F},{ }^{2} J \mathrm{C}-\mathrm{F}=25.0 \mathrm{~Hz}\right), 68.0,55.7$.

HRMS (ESI, $m / z$ ) calcd for $\mathrm{C}_{8} \mathrm{H}_{9} \mathrm{BFO}_{3}[\mathrm{M}+\mathrm{H}]^{+}: 183.0623$, found: 183.0614 .

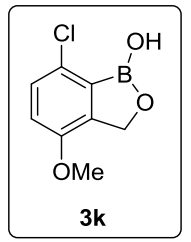

\section{7-chloro-4-methoxybenzo[c] [1,2] oxaborol-1(3H)-ol (3k)}

Conditions: 2-chloro-5-methoxyphenylboronic acid (186 mg, $1 \mathrm{mmol})$, paraformaldehyde (36 mg, 1.2 mmol), TfOH (60 mg, $35 \mu \mathrm{L}, 0.4$ equiv.), $\mathrm{CHCl}_{3}\left(10 \mathrm{~mL}, 40{ }^{\circ} \mathrm{C}, 36 \mathrm{~h}\right.$. The title product was obtained as white solid (130 mg, 66\%). m.p. $160.4-161.4{ }^{\circ} \mathrm{C} . R_{\mathrm{f}}=0.4$ (PE/EA, $\left.2 / 1\right)$.

${ }^{1} \mathrm{H}$ NMR (400 MHz, DMSO-d6): $\delta 9.18(\mathrm{~s}, 1 \mathrm{H}), 7.30(\mathrm{~d}, J=8.4 \mathrm{~Hz}, 1 \mathrm{H}), 7.07(\mathrm{~d}, J=8.5 \mathrm{~Hz}, 1 \mathrm{H}), 4.91$ (s, 2H), $3.82(\mathrm{~s}, 3 \mathrm{H})$.

${ }^{13} \mathrm{C}$ NMR (125 MHz, DMSO- $\left.d 6\right): \delta$ 152.6, 143.5, 128.9, 126.9, 114.3, 67.5, 55.6.

HRMS (ESI, $m / z$ ) calcd for $\mathrm{C}_{8} \mathrm{H}_{9} \mathrm{BClO}_{3}[\mathrm{M}+\mathrm{H}]^{+}: 199.0328$, found: 199.0330 .

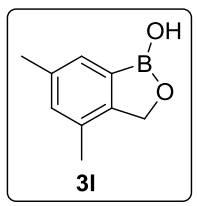

\section{4,6-dimethylbenzo[c] [1,2] oxaborol-1(3H)-ol (3I)}

Conditions: 3,5-dimethyphenylboronic acid $(105 \mathrm{mg}, 0.7 \mathrm{mmol})$, paraformaldehyde $(25 \mathrm{mg}, 0.84$ mmol), TfOH (11 mg, $6 \mu \mathrm{L}, 0.1$ equiv.), $\mathrm{CHCl}_{3}(7 \mathrm{~mL}), 50{ }^{\circ} \mathrm{C}, 72 \mathrm{~h}$. The title product was obtained as white solid (40 mg, 35\%). m.p. $132-132.5^{\circ} \mathrm{C} . R_{\mathrm{f}}=0.4$ (PE/EA, 5/1).

${ }^{1} \mathrm{H}$ NMR (400 MHz, DMSO-d6): $\delta 9.06$ (s, 1H), 7.34 (s, 1H), 7.07 (s, 1H), 4.90 (s, 2H), 2.31 (s, 3H), 2.18 (s, 3H).

${ }^{13} \mathrm{C}$ NMR (125 MHz, DMSO-d6): $\delta$ 150.1, 136.1, 132.4, 130.6, 128.2, 69.3, 21.0, 17.4.

HRMS (ESI, $m / z$ ) cacld for $\mathrm{C}_{9} \mathrm{H}_{12} \mathrm{BO}_{2}[\mathrm{M}+\mathrm{H}]^{+}: 163.0925$; found: 163.0928 .

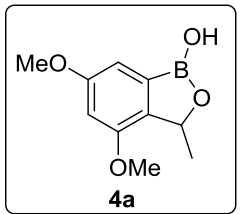

\section{4,6-dimethoxy-3-methylbenzo[c] [1,2] oxaborol-1(3H)-ol (4a)}

Conditions: 3,5-dimethoxyphenylboronic acid (127 mg, $0.7 \mathrm{mmol}$ ), acetaldehyde (37 mg, $47 \mu \mathrm{L}$, $1.2 \mathrm{mmol}$ ), TFA (319 mg, $208 \mu \mathrm{L}, 4$ equiv.), $\mathrm{CHCl}_{3}(7 \mathrm{~mL}), \mathrm{rt}, 53 \mathrm{~h}$. The title product was obtained as white solid (90 mg, 62\%). m.p. 110.1-110.8 ${ }^{\circ} \mathrm{C} . R_{\mathrm{f}}=0.4$ (PE/EA, 5/1).

${ }^{1} \mathrm{H}$ NMR (500 MHz, DMSO-d6): $\delta 9.04(\mathrm{~s}, 1 \mathrm{H}), 6.80(\mathrm{~s}, 1 \mathrm{H}), 6.62(\mathrm{~s}, 1 \mathrm{H}), 5.13(\mathrm{q}, J=6.3 \mathrm{~Hz}, 1 \mathrm{H}), 3.79(\mathrm{~s}, 3 \mathrm{H}), 3.78(\mathrm{~s}$, $3 \mathrm{H}), 1.38(\mathrm{~d}, J=6.4 \mathrm{~Hz}, 3 \mathrm{H})$. 
${ }^{13}$ C NMR (125 MHz, DMSO- $d 6$ ): $\delta$ 160.8, 154.6, 138.4, 104.9, 101.5, 75.2, 55.4, 55.4, 21.7.

HRMS (ESI, $m / z$ ) calcd for $\mathrm{C}_{10} \mathrm{H}_{14} \mathrm{BO}_{4}[\mathrm{M}+\mathrm{H}]^{+}$: 209.0980, found: 209.0982.

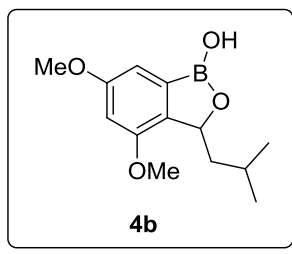

\section{3-isobutyl-4,6-dimethoxybenzo[c] [1,2] oxaborol-1(3H)-ol (4b)}

Conditions: 3,5-dimethoxyphenylboronic acid (182 mg, $1 \mathrm{mmol})$, isovaleraldehyde (103 $\mathrm{mg}$, $129 \mu \mathrm{L}, 1.2 \mathrm{mmol})$, TFA (570 mg, $371 \mu \mathrm{L}, 5.0$ equiv.), $\mathrm{CHCl}_{3}(10 \mathrm{~mL}), \mathrm{rt}, 18 \mathrm{~h}$. The title product was obtained as white solid $(188 \mathrm{mg}, 75 \%)$. m.p. $122.3-124.8{ }^{\circ} \mathrm{C} . R_{\mathrm{f}}=0.43(\mathrm{PE} / \mathrm{EA}$, $5 / 1)$.

${ }^{1} \mathrm{H}$ NMR (400 MHz, DMSO-d6): $\delta 9.04$ (s, $\left.1 \mathrm{H}\right), 6.79$ (d, $\left.J=1.8 \mathrm{~Hz}, 1 \mathrm{H}\right), 6.61(\mathrm{~d}, J=1.9 \mathrm{~Hz}, 1 \mathrm{H}), 5.10(\mathrm{dd}, J=9.8$, $2.2 \mathrm{~Hz}, 1 \mathrm{H}), 3.78(\mathrm{~s}, 3 \mathrm{H}), 3.77(\mathrm{~s}, 3 \mathrm{H}), 1.95-1.81(\mathrm{~m}, 2 \mathrm{H}), 1.20-1.14(\mathrm{~m}, 1 \mathrm{H}), 0.98(\mathrm{~d}, J=6.4 \mathrm{~Hz}, 3 \mathrm{H}), 0.87(\mathrm{~d}, J=6.4$ $\mathrm{Hz}, 3 \mathrm{H})$.

${ }^{13} \mathrm{C}$ NMR (125 MHz, DMSO- $\left.d 6\right): \delta$ 160.7, 154.6, 137.5, 104.8, 101.5, 77.6, 55.4, 55.4, 44.3, 24.8, 23.9, 22.0. HRMS (ESI, $m / z$ ) calcd for $\mathrm{C}_{13} \mathrm{H}_{20} \mathrm{BO}_{4}[\mathrm{M}+\mathrm{H}]^{+}: 251.1449$, found: 251.1450 .

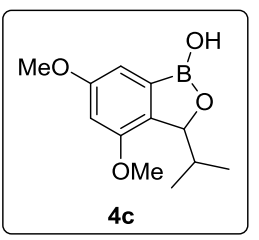

\section{3-isopropyl-4,6-dimethoxybenzo[c] [1,2] oxaborol-1(3H)-ol (4c)}

Conditions: 3,5-dimethoxyphenylboronic acid (182 mg, $1 \mathrm{mmol}$ ), isobutyraldehyde ( $86 \mathrm{mg}, 109$ $\mu \mathrm{L}, 1.2 \mathrm{mmol})$, TFA (684 mg, $446 \mu \mathrm{L}, 6$ equiv.), $\mathrm{CHCl}_{3}(10 \mathrm{~mL})$, rt, $24 \mathrm{~h}$. The title product was obtained as viscous liquid (216 mg, 92\%). $R_{\mathrm{f}}=0.42$ (PE/EA, 5/1).

${ }^{1} \mathrm{H}$ NMR $\left(400 \mathrm{MHz}, \mathrm{CDCl}_{3}\right): \delta 6.78(\mathrm{~d}, J=1.9 \mathrm{~Hz}, 1 \mathrm{H}), 6.53(\mathrm{~d}, J=2.0 \mathrm{~Hz}, 1 \mathrm{H}), 5.22(\mathrm{~d}, J=2.3$

$\mathrm{Hz}, 1 \mathrm{H}), 3.83$ (s, 3H), 3.80 (s, 3H), 2.57-2.49 (m, 1H), 1.19 (d, $J=7.0 \mathrm{~Hz}, 3 \mathrm{H}), 0.47$ (d, $J=6.9 \mathrm{~Hz}, 3 \mathrm{H})$.

${ }^{1} \mathrm{H}$ NMR (400 MHz, DMSO-d6): $\delta 9.05$ (s, 1H), 6.79 (d, $\left.J=1.9 \mathrm{~Hz}, 1 \mathrm{H}\right), 6.61(\mathrm{~d}, J=1.9 \mathrm{~Hz}, 1 \mathrm{H}), 5.03(\mathrm{~d}, J=2.2 \mathrm{~Hz}$, $1 \mathrm{H}), 3.78(\mathrm{~s}, 3 \mathrm{H}), 3.77$ (s, 3H), 2.46-2.38 (m, 1H), 1.09 (d, $J=6.9 \mathrm{~Hz}, 3 \mathrm{H}), 0.35$ (d, $J=6.8 \mathrm{~Hz}, 3 \mathrm{H})$.

${ }^{13} \mathrm{C}$ NMR (125 MHz, DMSO- $\left.d 6\right): \delta 160.8,154.7,135.9,104.7,101.4,83.1,55.4,55.3,29.7,20.4,13.8$.

HRMS (ESI, $m / z$ ) calcd for $\mathrm{C}_{12} \mathrm{H}_{18} \mathrm{BO}_{4}[\mathrm{M}+\mathrm{H}]^{+}: 237.1293$, found: 237.1300 .

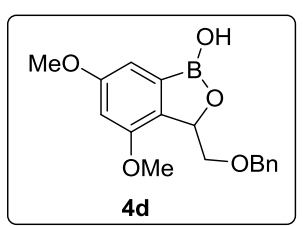

\section{3-(benzyloxy)-4,6-dimethoxybenzo[c] [1,2] oxaborol-1(3H)-ol (4d)}

Conditions: 3,5-dimethoxyphenylboronic acid (182 $\mathrm{mg}, 1 \mathrm{mmol})$, benzyloxyacetaldehyde (180 mg, $168 \mu \mathrm{L}, 1.2 \mathrm{mmol}$ ), TFA (456 mg, $297 \mu \mathrm{L}, 4$ equiv.), $\mathrm{CHCl}_{3}(10 \mathrm{~mL}), \mathrm{rt}, 24 \mathrm{~h}$. The title product was obtained as viscous liquid $(124 \mathrm{mg}, 41 \%) . R_{\mathrm{f}}=0.3$ (PE/EA, 5/1).

${ }^{1} \mathrm{H}$ NMR (400 MHz, DMSO-d6): $\delta 9.16$ (s, 1H), 7.35-7.31 (m, 2H), 7.29-7.24 (m, 3H), 6.81 (d, $J=1.8 \mathrm{~Hz}, 1 \mathrm{H}), 6.62(\mathrm{~d}, J=1.8 \mathrm{~Hz}, 1 \mathrm{H}), 5.23(\mathrm{dd}, J=6.1,1.7 \mathrm{~Hz}, 1 \mathrm{H}), 4.50(\mathrm{~d}, J=12.3 \mathrm{~Hz}, 1 \mathrm{H}), 4.45(\mathrm{~d}, J=12.3$ $\mathrm{Hz}, 1 \mathrm{H}), 4.00$ (dd, $J=10.7,1.8 \mathrm{~Hz}, 1 \mathrm{H}), 3.78(\mathrm{~s}, 3 \mathrm{H}), 3.76$ (s, 3H), 3.42 (dd, $J=10.7,6.1 \mathrm{~Hz}, 1 \mathrm{H})$.

${ }^{13}$ C NMR (125 MHz, DMSO- $d 6$ ): $\delta 161.2,154.8,138.6,133.4,128.3,127.5,127.4,105.0,101.4,79.0,72.4,71.8,55.5$, 55.4 .

HRMS (ESI, $m / z$ ) calcd for $\mathrm{C}_{17} \mathrm{H}_{19} \mathrm{BNaO}_{5}[\mathrm{M}+\mathrm{Na}]^{+}: 337.1218$, found: 337.1216 .

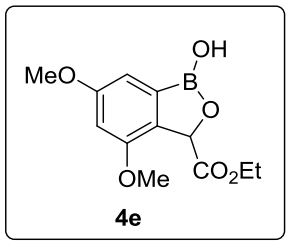

ethyl 1-hydroxy-4,6-dimethoxy-1,3-dihydrobenzo[c] [1,2] oxaborole-3-carboxylate (4e) Conditions: 3,5-dimethoxyphenylboronic acid $(182 \mathrm{mg}, 1 \mathrm{mmol})$, ethyl glyoxylate $(127 \mathrm{mg}$, $123 \mu \mathrm{L}, 1.2 \mathrm{mmol}$ ), TFA (34 mg, $22 \mu \mathrm{L}, 0.3$ equiv.), $\mathrm{CHCl}_{3}(10 \mathrm{~mL}), \mathrm{rt}, 72 \mathrm{~h}$. The title product was obtained as viscous liquid $(125 \mathrm{mg}, 47 \%) . R_{\mathrm{f}}=0.47$ (PE/EA, 2/1).

${ }^{1} \mathrm{H}$ NMR (400 MHz, DMSO-d6): $\delta 9.57(\mathrm{~s}, 1 \mathrm{H}), 6.85$ (d, $\left.J=1.9 \mathrm{~Hz}, 1 \mathrm{H}\right), 6.66(\mathrm{~d}, J=1.9 \mathrm{~Hz}$, 1H), $5.48(\mathrm{~s}, 1 \mathrm{H}), 4.19-4.07(\mathrm{~m}, 2 \mathrm{H}), 3.79(\mathrm{~s}, 3 \mathrm{H}), 3.77$ (s, 3H), $1.18(\mathrm{t}, J=7.1 \mathrm{~Hz}, 3 \mathrm{H})$.

${ }^{13} \mathrm{C}$ NMR (125 MHz, DMSO- $d 6$ ): $\delta$ 167.0, 161.8, 155.0, 132.8, 105.3, 101.8, 77.5, 60.9, 55.6, 55.5, 14.1.

HRMS (ESI, $m / z$ ) calcd for $\mathrm{C}_{12} \mathrm{H}_{16} \mathrm{BO}_{6}[\mathrm{M}+\mathrm{H}]^{+}: 267.1034$, found: 267.1038 . 


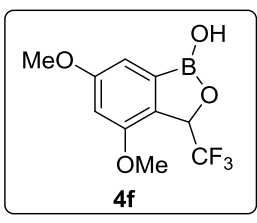

\section{4,6-dimethoxy-3-(trifluoromethyl) benzo[c] [1,2] oxaborol-1(3H)-ol (4f)}

Conditions: 3,5-dimethoxyphenylboronic acid (182 $\mathrm{mg}, 1 \mathrm{mmol})$, trifluoracetaldehyde monohydrate (75 wt.\% aqueous) (186 mg, $1.2 \mathrm{mmol})$, TFA (1140 mg, $743 \mu \mathrm{L}, 10$ equiv.), $\mathrm{CHCl}_{3}$ $(10 \mathrm{~mL}), \mathrm{rt}, 33 \mathrm{~h}$. The title product was obtained as white solid (234 mg, 89\%). m.p. 121.6-121.9 ${ }^{\circ} \mathrm{C} . R_{\mathrm{f}}=0.35(\mathrm{PE} / \mathrm{EA}, 5 / 1)$.

${ }^{1} \mathrm{H}$ NMR (400 MHz, DMSO-d6): $\delta 9.87(\mathrm{~s}, 1 \mathrm{H}), 6.89(\mathrm{~d}, J=1.9 \mathrm{~Hz}, 1 \mathrm{H}), 6.73(\mathrm{~d}, J=1.9 \mathrm{~Hz}, 1 \mathrm{H}), 5.72(\mathrm{q}, J=5.7 \mathrm{~Hz}$, $1 \mathrm{H}), 3.81(\mathrm{~s}, 6 \mathrm{H})$.

${ }^{13} \mathrm{C}$ NMR (125 MHz, DMSO-d6): $\delta$ 162.5, 156.0, 127.0, 124.5 (q, C-F, ${ }^{1} J$ C-F = 280.0 Hz), 105.8, 102.3, 75.8 (q, C-F, ${ }^{2} J$ $\mathrm{C}-\mathrm{F}=32.8 \mathrm{~Hz}), 55.7,55.6$.

HRMS (ESI, $m / z$ ) calcd for $\mathrm{C}_{10} \mathrm{H}_{9} \mathrm{BF}_{3} \mathrm{O}_{4}[\mathrm{M}-\mathrm{H}]$ : 261.0551 , found: 261.0547.

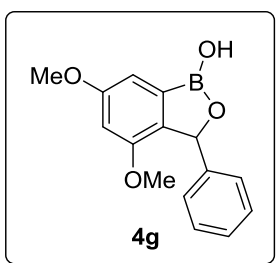

${ }^{13} \mathrm{C}$ NMR (125 MHz, DMSO-d6): $\delta$ 161.3, 154.9, 140.9, 136.6, 128.1, 127.7, 127.1, 104.9, 101.8, 80.5, 55.5, 55.3.

\section{4,6-dimethoxy-3-phenylbenzo[c] [1,2] oxaborol-1(3H)-ol (4g)}

Conditions: 3,5-dimethoxyphenylboronic acid (91 mg, $0.5 \mathrm{mmol})$, benzaldehyde $(64 \mathrm{mg}, 61 \mu \mathrm{L}$ $0.6 \mathrm{mmol})$, TFA (285 mg, $186 \mu \mathrm{L}, 5$ equiv. $), \mathrm{CHCl}_{3}(5 \mathrm{~mL}), \mathrm{rt}, 44 \mathrm{~h}$. The title product was obtained as white solid (69 mg, 51\%). m.p. $167.5-168.1{ }^{\circ} \mathrm{C} . R_{\mathrm{f}}=0.38(\mathrm{PE} / \mathrm{EA}, 5 / 1)$

${ }^{1} \mathrm{H}$ NMR (400 MHz, DMSO-d6): $\delta 9.28(\mathrm{~s}, 1 \mathrm{H}), 7.31-7.25(\mathrm{~m}, 3 \mathrm{H}), 7.18-7.13(\mathrm{~m}, 2 \mathrm{H}), 6.89(\mathrm{~d}$, $J=2.0 \mathrm{~Hz}, 1 \mathrm{H}), 6.59(\mathrm{~d}, J=2.0 \mathrm{~Hz}, 1 \mathrm{H}), 6.04(\mathrm{~s}, 1 \mathrm{H}), 3.79(\mathrm{~s}, 3 \mathrm{H}), 3.59(\mathrm{~s}, 3 \mathrm{H})$.

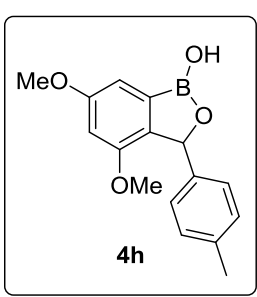

$2.26(\mathrm{~s}, 3 \mathrm{H})$

${ }^{13} \mathrm{C}$ NMR (125 MHz, DMSO-d6): $\delta$ 161.2, 154.9, 137.9, 136.8, 136.7, 128.7, 127.0, 104.8, 101.8, 80.4, 55.4, 55.3, 20.8. HRMS (ESI, $m / z$ ) calcd for $\mathrm{C}_{16} \mathrm{H}_{18} \mathrm{BO}_{4}[\mathrm{M}+\mathrm{H}]^{+}$: 285.1293, found: 285.1296

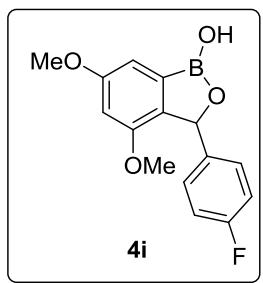

3-(4-fluorophenyl)-4,6-dimethoxybenzo[c] [1,2] oxaborol-1(3H)-ol (4i)

Conditions: 3,5-dimethoxyphenylboronic acid (127 mg, $0.7 \mathrm{mmol})$, 4-fluorobenzaldehyde (104 mg, $90 \mu \mathrm{L}, 0.84 \mathrm{mmol})$, TFA (399 mg, $260 \mu \mathrm{L}, 5$ equiv.), $\mathrm{CHCl}_{3}$ (7 mL), rt, 40 h. The title product was obtained as white solid (114 mg, 51\%). m.p. $154.9-157.1{ }^{\circ} \mathrm{C} . R_{\mathrm{f}}=0.4(\mathrm{PE} / \mathrm{EA}, 5 / 1)$.

${ }^{1} \mathrm{H}$ NMR (400 MHz, DMSO-d6): $\delta 9.31$ (s, 1H), 7.21-7.16 (m, 2H), 7.14-7.08 (m, 2H), 6.88 (d, J $=2.0 \mathrm{~Hz}, 1 \mathrm{H}), 6.60(\mathrm{~d}, J=2.0 \mathrm{~Hz}, 1 \mathrm{H}), 6.05(\mathrm{~s}, 1 \mathrm{H}), 3.79(\mathrm{~s}, 3 \mathrm{H}), 3.60(\mathrm{~s}, 3 \mathrm{H})$.

${ }^{13} \mathrm{C}$ NMR (125 MHz, DMSO-d6): $\delta 161.7\left(\mathrm{~d}, \mathrm{C}-\mathrm{F},{ }^{1} J \mathrm{C}-\mathrm{F}=242.7 \mathrm{~Hz}\right), 161.3,154.3,137.1\left(\mathrm{~d}, \mathrm{C}-\mathrm{F},{ }^{4} J \mathrm{C}-\mathrm{F}=2.9 \mathrm{~Hz}\right)$, $136.4,129.1\left(\mathrm{~d}, \mathrm{C}-\mathrm{F},{ }^{3} J \mathrm{C}-\mathrm{F}=8.3 \mathrm{~Hz}\right), 114.9\left(\mathrm{~d}, \mathrm{C}-\mathrm{F},{ }^{2} J \mathrm{C}-\mathrm{F}=21.4 \mathrm{~Hz}\right), 105.0,102.0,79.7,55.5,55.4$.

HRMS (ESI, $m / z$ ) calcd for $\mathrm{C}_{15} \mathrm{H}_{15} \mathrm{BFO}_{4}[\mathrm{M}+\mathrm{H}]^{+}$: 289.1042, found: 289.1047 .

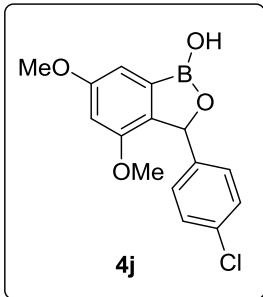

3-(4-chlorophenyl)-4,6-dimethoxybenzo[c] [1,2] oxaborol-1(3H)-ol (4j)

Conditions: 3,5-dimethoxyphenylboronic acid (127 mg, $0.7 \mathrm{mmol})$, 4-chlorobenzaldehyde (118 mg, $0.84 \mathrm{mmol}$ ), TFA (399 mg, $260 \mu \mathrm{L}, 5$ equiv.), $\mathrm{CHCl}_{3}(7 \mathrm{~mL}), \mathrm{rt}, 40 \mathrm{~h}$. The title product was 
obtained as white solid (183 mg, 86\%). m.p. 149.6-150.6 ${ }^{\circ} \mathrm{C} . R_{\mathrm{f}}=0.38$ (PE/EA, 5/1). When the reaction was carried out in $5 \mathrm{mmol}$ scale, the loading amount of TFA was reduced to 4 equivalents, and $1429 \mathrm{mg}(94 \%)$ of $\mathbf{4 j}$ was obtained.

${ }^{1} \mathrm{H}$ NMR (400 MHz, DMSO-d6): $\delta 9.35(\mathrm{~s}, 1 \mathrm{H}), 7.36$ (d, $\left.J=8.4 \mathrm{~Hz}, 2 \mathrm{H}\right), 7.18(\mathrm{~d}, J=8.4 \mathrm{~Hz}, 2 \mathrm{H}), 6.89$ (d, $J=1.9 \mathrm{~Hz}$, $1 \mathrm{H}), 6.60(\mathrm{~d}, J=1.9 \mathrm{~Hz}, 1 \mathrm{H}), 6.05(\mathrm{~s}, 1 \mathrm{H}), 3.79(\mathrm{~s}, 3 \mathrm{H}), 3.61(\mathrm{~s}, 3 \mathrm{H})$.

${ }^{13} \mathrm{C}$ NMR (125 MHz, DMSO- $\left.d 6\right): \delta 161.4,154.8,139.9,136.2,132.2,128.9,128.2,105.0,101.9,79.6,55.5,55.3$. HRMS (ESI, $m / z$ ) calcd for $\mathrm{C}_{15} \mathrm{H}_{15} \mathrm{BClO}_{4}[\mathrm{M}+\mathrm{H}]^{+}: 305.0746$, found: 305.0749 .

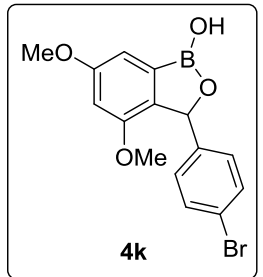

\section{3-(4-bromophenyl)-4,6-dimethoxybenzo[c] [1,2] oxaborol-1(3H)-ol (4k)}

Conditions: 3,5-dimethoxyphenylboronic acid (91 mg, $0.5 \mathrm{mmol})$, 4-bromobenzaldehyde (111 $\mathrm{mg}, 0.6 \mathrm{mmol})$, TFA (228 mg, $149.0 \mu \mathrm{L}, 4$ equiv.), $\mathrm{CHCl}_{3}(5 \mathrm{~mL}), \mathrm{rt}, 42 \mathrm{~h}$. The title product was obtained as white solid (100 mg, 57\%). m.p. $154.3-155.6{ }^{\circ} \mathrm{C} . R_{\mathrm{f}}=0.38(\mathrm{PE} / \mathrm{EA}, 5 / 1)$.

${ }^{1} \mathrm{H}$ NMR (500 MHz, DMSO-d6): $\delta 9.35$ (s, 1H), 7.49 (d, $\left.J=8.4 \mathrm{~Hz}, 2 \mathrm{H}\right), 7.12$ (d, $J=8.4 \mathrm{~Hz}$, 2H), $6.89(\mathrm{~d}, J=1.9 \mathrm{~Hz}, 1 \mathrm{H}), 6.60(\mathrm{~d}, J=1.9 \mathrm{~Hz}, 1 \mathrm{H}), 6.04(\mathrm{~s}, 1 \mathrm{H}), 3.79(\mathrm{~s}, 3 \mathrm{H}), 3.61(\mathrm{~s}, 3 \mathrm{H})$.

${ }^{13} \mathrm{C}$ NMR (125 MHz, DMSO-d6): $\delta$ 161.4, 154.8, 140.3, 136.1, 131.1, 129.3, 120.8, 105.0, 101.9, 79.7, 55.5, 55.4.

HRMS (ESI, $m / z$ ) calcd for $\mathrm{C}_{15} \mathrm{H}_{15} \mathrm{BBrO}_{4}[\mathrm{M}+\mathrm{H}]^{+}: 349.0241$, found: 349.0239 .

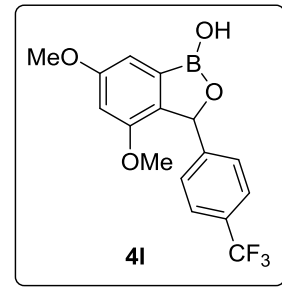

4,6-dimethoxy-3-(4-(trifluoromethyl) phenyl) benzo[c] [1,2] oxaborol-1(3H)-ol (4I)

Conditions: 3,5-dimethoxyphenylboronic acid (182 mg, $1 \mathrm{mmol})$, 4-(trifluoromethy) benzaldehyde (209 mg, $164 \mu \mathrm{L}, 1.2 \mathrm{mmol})$, TFA (456 mg, $297 \mu \mathrm{L}, 4$ equiv.), $\mathrm{CHCl}_{3}(10 \mathrm{~mL})$, rt, 24 h. The title product was obtained as white solid (230 mg, 68\%). m.p. $144.7-146.6{ }^{\circ} \mathrm{C} . R_{\mathrm{f}}$ $=0.40(\mathrm{PE} / \mathrm{EA}, 5 / 1)$.

${ }^{1} \mathrm{H}$ NMR (400 MHz, DMSO-d6): $\delta 9.44(\mathrm{~s}, 1 \mathrm{H}), 7.68$ (d, $\left.J=8.2 \mathrm{~Hz}, 2 \mathrm{H}\right), 7.41$ (d, $J=8.1 \mathrm{~Hz}$, 2H), $6.91(\mathrm{~d}, J=1.9 \mathrm{~Hz}, 1 \mathrm{H}), 6.61(\mathrm{~d}, J=1.9 \mathrm{~Hz}, 1 \mathrm{H}), 6.16(\mathrm{~s}, 1 \mathrm{H}), 3.80(\mathrm{~s}, 3 \mathrm{H}), 3.62(\mathrm{~s}, 3 \mathrm{H})$.

${ }^{13} \mathrm{C}$ NMR (125 MHz, DMSO-d6): $\delta$ 161.6, 154.9, 145.5, 136.1, 128.5 (q, d, C-F, ${ }^{2} J$ C-F = 31.5 Hz), 127.9, 125.1 (q, C-F, $\left.{ }^{3} J \mathrm{C}-\mathrm{F}=3.7 \mathrm{~Hz}\right), 124.4\left(\mathrm{q}, \mathrm{C}-\mathrm{F},{ }^{1} J \mathrm{C}-\mathrm{F}=270.4 \mathrm{~Hz}\right), 105.2,102.0,79.8,55.5,55.3$.

HRMS (ESI, $m / z$ ) calcd for $\mathrm{C}_{16} \mathrm{H}_{15} \mathrm{BF}_{3} \mathrm{O}_{4}[\mathrm{M}+\mathrm{H}]^{+}: 339.1010$, found: 339.1015 .

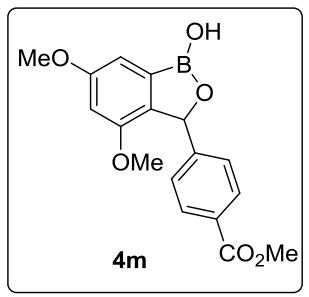

methyl 4-(1-hydroxy-4,6-dimethoxy-1,3-dihydrobenzo[c] [1,2] oxaborol-3-yl) benzoate $(4 \mathrm{~m})$

Conditions: 3,5-dimethoxyphenylboronic acid (127 mg, $0.7 \mathrm{mmol})$, methyl 4-formylbenzoate (138 mg, $0.84 \mathrm{mmol}$ ), TFA (160 mg, $104 \mu \mathrm{L}, 2$ equiv.), $\mathrm{CHCl}_{3}(7 \mathrm{~mL}), \mathrm{rt}, 24 \mathrm{~h}$. The title product was obtained as white solid $(65 \mathrm{mg}, 28 \%)$. m.p. $175.5-177.1^{\circ} \mathrm{C} . R_{\mathrm{f}}=0.5$ (PE/EA, $5 / 1)$.

${ }^{1} \mathrm{H}$ NMR (500 MHz, DMSO-d6): $\delta 9.39(\mathrm{~s}, 1 \mathrm{H}), 7.90(\mathrm{~d}, J=8.2 \mathrm{~Hz}, 2 \mathrm{H}), 7.32(\mathrm{~d}, J=8.2 \mathrm{~Hz}, 2 \mathrm{H}), 6.89(\mathrm{~d}, J=1.7 \mathrm{~Hz}$, $1 \mathrm{H}), 6.59(\mathrm{~d}, J=1.8 \mathrm{~Hz}, 1 \mathrm{H}), 6.12(\mathrm{~s}, 1 \mathrm{H}), 3.83(\mathrm{~s}, 3 \mathrm{H}), 3.79(\mathrm{~s}, 3 \mathrm{H}), 3.59$ (s, 3H).

${ }^{13} \mathrm{C}$ NMR (125 MHz, DMSO- $d 6$ ): $\delta$ 166.2, 161.4, 154.8, 146.1, 136.1, 129.1, 129.1, 127.4, 105.1, 101.9, 79.9, 55.5, 55.3, 52.1 .

HRMS (ESI, $m / z$ ) calcd for $\mathrm{C}_{17} \mathrm{H}_{18} \mathrm{BO}_{6}[\mathrm{M}+\mathrm{H}]^{+}: 329.1191$, found: 329.1199 .

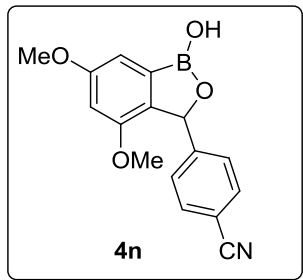

4-(1-hydroxy-4,6-dimethoxy-1,3-dihydrobenzo[c] [1,2] oxaborol-3-yl) benzonitrile (4n) Conditions: 3,5-dimethoxyphenylboronic acid (182 mg, $1 \mathrm{mmol})$, 4-cyanobenzaldehyde (157 mg, $1.2 \mathrm{mmol})$, TFA ( $684 \mathrm{mg}, 446 \mu \mathrm{L}, 6$ equiv.), $\mathrm{CHCl}_{3}(10 \mathrm{~mL}), \mathrm{rt}, 60 \mathrm{~h}$. The title product was obtained as white solid ( $251 \mathrm{mg}, 85 \%$ ). m.p. $223.3-226.0^{\circ} \mathrm{C} . R_{\mathrm{f}}=0.32$ (PE/EA, $\left.2 / 1\right)$. ${ }^{1} \mathrm{H}$ NMR (400 MHz, DMSO- $\left.d 6\right): \delta 9.46(\mathrm{~s}, 1 \mathrm{H}), 7.78(\mathrm{~d}, J=8.3 \mathrm{~Hz}, 2 \mathrm{H}), 7.38(\mathrm{~d}, J=8.3 \mathrm{~Hz}$, 
2H), $6.90(\mathrm{~d}, J=1.9 \mathrm{~Hz}, 1 \mathrm{H}), 6.60(\mathrm{~d}, J=1.9 \mathrm{~Hz}, 1 \mathrm{H}), 6.14(\mathrm{~s}, 1 \mathrm{H}), 3.79(\mathrm{~s}, 3 \mathrm{H}), 3.61(\mathrm{~s}, 3 \mathrm{H})$.

${ }^{13} \mathrm{C}$ NMR (125 MHz, DMSO-d6): $\delta$ 161.6, 154.8, 146.3, 135.8, 132.2, 128.1, 118.9, 110.6, 105.2, 102.0, 79.7, 55.5, 55.4. HRMS (ESI, $m / z$ ) calcd for $\mathrm{C}_{16} \mathrm{H}_{15} \mathrm{BNO}_{4}[\mathrm{M}+\mathrm{H}]^{+}: 296.1089$, found: 296.1091 .

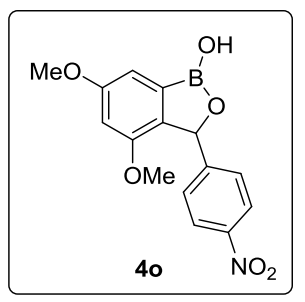

\section{4,6-dimethoxy-3-(4-nitrophenyl) benzo[c] [1,2] oxaborol-1(3H)-ol (4o)}

Conditions: 3,5-dimethoxyphenylboronic acid (91 mg, $0.5 \mathrm{mmol})$, 4-nitrobenzaldehyde (91 mg, $0.6 \mathrm{mmol})$, TFA (1570 mg, $371 \mu \mathrm{L}, 10$ equiv.), $\mathrm{CHCl}_{3}(5 \mathrm{~mL})$, rt, $48 \mathrm{~h}$. The title product was obtained as white solid (120 mg, 76\%). m.p. $237.9-240.9^{\circ} \mathrm{C} . R_{\mathrm{f}}=0.3$ (PE/EA,2/1).

${ }^{1} \mathrm{H}$ NMR (400 MHz, DMSO-d6): $\delta 9.47$ (s, 1H), $8.18(\mathrm{~d}, J=8.7 \mathrm{~Hz}, 2 \mathrm{H}), 7.48(\mathrm{~d}, J=8.7 \mathrm{~Hz}$, $2 \mathrm{H}), 6.92(\mathrm{~d}, J=1.6 \mathrm{~Hz}, 1 \mathrm{H}), 6.61(\mathrm{~d}, J=1.7 \mathrm{~Hz}, 1 \mathrm{H}), 6.21(\mathrm{~s}, 1 \mathrm{H}), 3.80(\mathrm{~s}, 3 \mathrm{H}), 3.62(\mathrm{~s}, 3 \mathrm{H})$.

${ }^{13} \mathrm{C}$ NMR (125 MHz, DMSO- $\left.d 6\right): \delta 161.6,154.8,148.3,147.1,135.7,128.3,123.4,105.2,102.0,79.3,55.5,55.4$.

HRMS (ESI, $m / z$ ) calcd for $\mathrm{C}_{15} \mathrm{H}_{15} \mathrm{BNO}_{6}[\mathrm{M}+\mathrm{H}]^{+}: 316.0987$, found: 316.0985 .

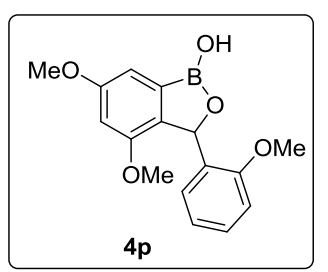

\section{4,6-dimethoxy-3-(2-methoxyphenyl) benzo [c] [1,2] oxaborol-1(3H)-ol (4p)}

Conditions: 3,5-dimethoxyphenylboronic acid (182 mg, $1 \mathrm{mmol})$, 2-methoxybenzaldehyde (163 mg, $1.2 \mathrm{mmol})$, TFA (1710 mg, $1114 \mu \mathrm{L}, 15$ equiv.), $\mathrm{CHCl}_{3}(10 \mathrm{~mL})$, rt, $18 \mathrm{~h}$. The title product was obtained as white solid $(75 \mathrm{mg}, 25 \%)$ m.p. $191.6-192.1{ }^{\circ} \mathrm{C} . R_{\mathrm{f}}=0.4$ (PE/EA, $5 / 1)$.

${ }^{1} \mathrm{H}$ NMR (500 MHz, DMSO-d6): $\delta 9.13$ (s, 1H), $7.27-7.22(\mathrm{~m}, 1 \mathrm{H}), 7.01$ (d, $J=8.1 \mathrm{~Hz}$, $1 \mathrm{H}), 6.89(\mathrm{~d}, J=1.9 \mathrm{~Hz}, 1 \mathrm{H}), 6.80-6.76(\mathrm{~m}, 1 \mathrm{H}), 6.61-6.59(\mathrm{~m}, 2 \mathrm{H}), 6.39(\mathrm{~s}, 1 \mathrm{H}), 3.83(\mathrm{~s}, 3 \mathrm{H}), 3.81(\mathrm{~s}, 3 \mathrm{H}), 3.58(\mathrm{~s}$, $3 \mathrm{H})$.

${ }^{13}$ C NMR (125 MHz, DMSO-d6): $\delta$ 161.2, 157.6, 154.8, 136.0, 129.2, 128.7, 127.1, 120.3, 111.5, 104.9, 101.8, 74.5, 55.8, $55.5,55.3$.

HRMS (ESI, $m / z$ ) calcd for $\mathrm{C}_{16} \mathrm{H}_{18} \mathrm{BO}_{5}[\mathrm{M}+\mathrm{H}]^{+}: 301.1242$, found: 301.1245 .

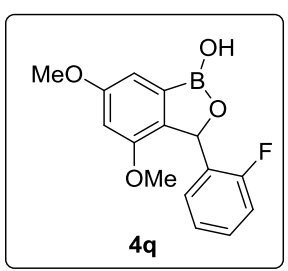

\section{3-(2-fluorophenyl)-4,6-dimethoxybenzo[c] [1,2] oxaborol-1(3H)-ol (4q)}

Conditions: 3,5-dimethoxyphenylboronic acid (182 mg, $1 \mathrm{mmol})$, 2-fluorobenzaldehyde (149 $\mathrm{mg}, 126 \mu \mathrm{L}, 1.2 \mathrm{mmol})$, TFA (342 mg, $223 \mu \mathrm{L}, 3$ equiv.), $\mathrm{CHCl}_{3}(10 \mathrm{~mL}), \mathrm{rt}, 96 \mathrm{~h}$. The title product was obtained as white solid $(178 \mathrm{mg}, 62 \%)$. m.p. $170.9-172.8{ }^{\circ} \mathrm{C} . R_{\mathrm{f}}=0.35$ (PE/EA, $5 / 1)$.

${ }^{1} \mathrm{H}$ NMR (500 MHz, DMSO-d6): $\delta 9.32(\mathrm{~s}, 1 \mathrm{H}), 7.35-7.31(\mathrm{~m}, 1 \mathrm{H}), 7.19$ - $7.15(\mathrm{~m}, 1 \mathrm{H}), 7.10$ $7.07(\mathrm{~m}, 1 \mathrm{H}), 6.92-6.90(\mathrm{~m}, 2 \mathrm{H}), 6.62(\mathrm{~s}, 1 \mathrm{H}), 6.26(\mathrm{~s}, 1 \mathrm{H}), 3.80(\mathrm{~s}, 3 \mathrm{H}), 3.59(\mathrm{~s}, 3 \mathrm{H})$.

${ }^{13} \mathrm{C}$ NMR (125 MHz, DMSO-d6): $\delta 160.7\left(\mathrm{~d}, \mathrm{C}-\mathrm{F},{ }^{1} J \mathrm{C}-\mathrm{F}=245.5 \mathrm{~Hz}\right), 161.5,154.8,135.4,129.9\left(\mathrm{~d}, \mathrm{C}-\mathrm{F},{ }^{3} J \mathrm{C}-\mathrm{F}=8.3\right.$ $\mathrm{Hz}), 128.7$ (d, C-F, $\left.{ }^{4} J \mathrm{C}-\mathrm{F}=3.8 \mathrm{~Hz}\right), 127.7\left(\mathrm{~d}, \mathrm{C}-\mathrm{F},{ }^{3} J \mathrm{C}-\mathrm{F}=12.7 \mathrm{~Hz}\right), 124.3\left(\mathrm{~d}, \mathrm{C}-\mathrm{F},{ }^{4} J \mathrm{C}-\mathrm{F}=3.1 \mathrm{~Hz}\right), 115.5\left(\mathrm{~d}, \mathrm{C}-\mathrm{F},{ }^{2} J\right.$ $\mathrm{C}-\mathrm{F}=21.7 \mathrm{~Hz}), 105.1,101.8,74.4\left(\mathrm{~d}, \mathrm{C}-\mathrm{F},{ }^{3} J \mathrm{C}-\mathrm{F}=3.3 \mathrm{~Hz}\right), 55.5,55.4$.

HRMS (ESI, $m / z$ ) calcd for $\mathrm{C}_{15} \mathrm{H}_{15} \mathrm{BFO}_{4}[\mathrm{M}+\mathrm{H}]^{+}: 289.1042$, found: 289.1044 .

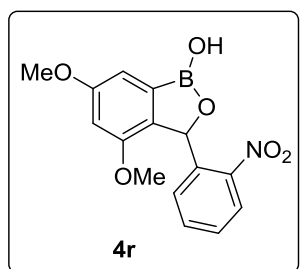

(s, 3H), 3.57 (s, 3H).

\section{4,6-dimethoxy-3-(2-nitrophenyl) benzo[c] [1,2] oxaborol-(3H)-ol (4r)}

Conditions: 3,5-dimethoxyphenylboronic acid (91 mg, $0.5 \mathrm{mmol})$, 2-nitrobenzaldehyde (91 $\mathrm{mg}, 0.6 \mathrm{mmol}$ ), TFA (456 mg, $297 \mu \mathrm{L}, 8$ equiv.), $\mathrm{CHCl}_{3}(5 \mathrm{~mL}), \mathrm{rt}, 24 \mathrm{~h}$. The title product was obtained as brown solid (82 mg, 52\%). m.p. $164.7-167.3{ }^{\circ}$ C. $R_{\mathrm{f}}=0.41$ (PE/EA, 2/1). ${ }^{1} \mathrm{H}$ NMR (400 MHz, DMSO-d6): $\delta 9.55(\mathrm{~s}, 1 \mathrm{H}), 7.92(\mathrm{~d}, J=7.9 \mathrm{~Hz}, 1 \mathrm{H}), 7.61-7.50(\mathrm{~m}, 2 \mathrm{H})$, $7.10(\mathrm{~d}, J=6.9 \mathrm{~Hz}, 1 \mathrm{H}), 6.89(\mathrm{~d}, J=1.8 \mathrm{~Hz}, 1 \mathrm{H}), 6.74(\mathrm{~s}, 1 \mathrm{H}), 6.59(\mathrm{~d}, J=1.8 \mathrm{~Hz}, 1 \mathrm{H}), 3.79$ 
${ }^{13}$ C NMR (125 MHz, DMSO- $\left.d 6\right): \delta 161.7,154.5,149.4,135.5,133.8,133.1,129.1,127.9,124.2,105.3,101.9,74.4,55.5$, 55.3.

HRMS (ESI, $m / z$ ) calcd for $\mathrm{C}_{15} \mathrm{H}_{15} \mathrm{BNO}_{6}[\mathrm{M}+\mathrm{H}]^{+}: 316.0987$, found: 316.0990 .

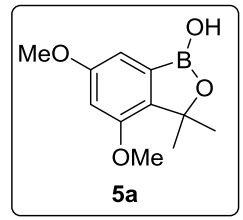

\section{4,6-dimethoxy-3,3-dimethylbenzo[c] [1,2] oxaborol-1(3H)-ol (5a)}

Conditions for ketone: 3,5-dimethoxyphenylboronic acid (91 mg, $0.5 \mathrm{mmol})$, acetone $(58 \mathrm{mg}, 73$ $\mu \mathrm{L}, 1 \mathrm{mmol}$ ), TfOH (30 mg, $18 \mu \mathrm{L}, 0.4$ equiv.), $\mathrm{CHCl}_{3}(5 \mathrm{~mL}), \mathrm{rt}, 24 \mathrm{~h}$. The title product was obtained as white solid $(77 \mathrm{mg}, 70 \%)$.

Conditions for ketal: 3,5-dimethoxyphenylboronic acid $(182 \mathrm{mg}, 1 \mathrm{mmol})$, 2,2-dimethoxypropane (208 mg, $246 \mu \mathrm{L}, 2 \mathrm{mmol}$ ), TfOH (150 mg, $88 \mu \mathrm{L}, 1$ equiv.), $\mathrm{CHCl}_{3}(10 \mathrm{~mL})$, rt, $48 \mathrm{~h}$. The title product was obtained as white solid $(220 \mathrm{mg}, 99 \%)$. When the reaction was carried out in $7 \mathrm{mmol}$ scale, the loading amount of TfOH was increased to 1.7 equivalents, and $1501 \mathrm{mg}(97 \%)$ of $\mathbf{5 a}$ was obtained. m.p. $163 \cdot 4-164 \cdot 7^{\circ} \mathrm{C} \cdot R_{\mathrm{f}}=0.45$ (PE/EA, 2/1).

${ }^{1} \mathrm{H}$ NMR (400 MHz, DMSO-d6): $\delta 8.94(\mathrm{~s}, 1 \mathrm{H}), 6.77$ (d, $\left.J=1.9 \mathrm{~Hz}, 1 \mathrm{H}\right), 6.61(\mathrm{~d}, J=1.8 \mathrm{~Hz}, 1 \mathrm{H}), 3.80$ (s, 3H), 3.77 (s, $3 \mathrm{H}), 1.45(\mathrm{~s}, 6 \mathrm{H})$

${ }^{13} \mathrm{C}$ NMR (125 MHz, DMSO- $\left.d 6\right): \delta 160.8,154.4,141.5,105.0,101.8,82.3,55.5,55.4,27.7$.

HRMS (ESI, $m / z$ ) calcd for $\mathrm{C}_{11} \mathrm{H}_{16} \mathrm{BO}_{4}[\mathrm{M}+\mathrm{H}]^{+}: 223.1136$, found: 223.1139 .

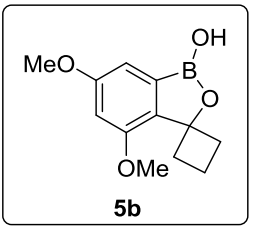

\section{4,6-dimethoxy-1H-spiro[benzo[c] [1,2] oxaborole-3,1'-cyclobutan]-1-ol (5b)}

Conditions: 3,5-dimethoxyphenylboronic acid (182 mg, $1 \mathrm{mmol})$, cyclobutanone ( $84 \mathrm{mg}, 90 \mu \mathrm{L}$, $1.2 \mathrm{mmol}), \mathrm{TfOH}(60 \mathrm{mg}, 35 \mu \mathrm{L}, 0.4$ equiv. $), \mathrm{CHCl}_{3}(10 \mathrm{~mL}), \mathrm{rt}, 15 \mathrm{~h}$. The title product was obtained as white solid (143 mg, 61\%). m.p. $117.4-117.6{ }^{\circ} \mathrm{C} . R_{\mathrm{f}}=0.41$ (PE/EA, 5/1).

${ }^{1} \mathrm{H}$ NMR (400 MHz, DMSO- $\left.d 6\right): \delta 9.06(\mathrm{~s}, 1 \mathrm{H}), 6.74(\mathrm{~d}, J=2.0 \mathrm{~Hz}, 1 \mathrm{H}), 6.65(\mathrm{~d}, J=2.0 \mathrm{~Hz}$, $1 \mathrm{H}), 3.87$ (s, 3H), 3.77 (s, 3H), 2.74-2.65 (m, 2H), 2.27-2.17 (m, 2H), 2.07-1.99 (m, 2H).

${ }^{13} \mathrm{C}$ NMR (125 MHz, DMSO- $\left.d 6\right): \delta 160.8,155.3,138.4,104.6,101.8,85.7,55.6,55.4,33.3,13.3$.

HRMS (ESI, $m / z$ ) calcd for $\mathrm{C}_{12} \mathrm{H}_{16} \mathrm{BO}_{4}[\mathrm{M}+\mathrm{H}]^{+}:$235.1136, found: 235.1148 .

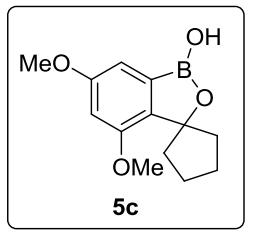

\section{4,6-dimethoxy-1H-spiro[benzo[c] [1,2] oxaborole-3,1'-cyclopentan]-1-ol (5c)}

Conditions: 3,5-dimethoxyphenylboronic acid (182 mg, $1 \mathrm{mmol})$, cyclopentanone (101 mg, 106 $\mu \mathrm{L}, 1.2 \mathrm{mmol})$, TfOH (150 mg, $88 \mu \mathrm{L}, 1$ equiv.), $\mathrm{CHCl}_{3}(10 \mathrm{~mL})$, rt, $11 \mathrm{~h}$. The title product was obtained as white solid (223 mg, 90\%). m.p. $150 \cdot 6-152.7{ }^{\circ} \mathrm{C} . R_{\mathrm{f}}=0.41$ (PE/EA, 5/1).

${ }^{1} \mathrm{H}$ NMR (500 MHz, DMSO-d6): $\delta 8.93(\mathrm{~s}, 1 \mathrm{H}), 6.78(\mathrm{~d}, J=1.9 \mathrm{~Hz}, 1 \mathrm{H}), 6.62(\mathrm{~d}, J=1.9 \mathrm{~Hz}, 1 \mathrm{H})$, $3.80(\mathrm{~s}, 3 \mathrm{H}), 3.78(\mathrm{~s}, 3 \mathrm{H}), 2.32-2.24(\mathrm{~m}, 2 \mathrm{H}), 1.90-1.78(\mathrm{~m}, 4 \mathrm{H}), 1.52-1.46(\mathrm{~m}, 2 \mathrm{H})$.

${ }^{13} \mathrm{C}$ NMR (125 MHz, DMSO-d6): $\delta$ 160.8, 154.6, 137.8, 104.7, 101.8, 92.5, 79.3, 55.5, 55.4, 37.7, 24.5.

HRMS (ESI, $m / z$ ) calcd for $\mathrm{C}_{13} \mathrm{H}_{18} \mathrm{BO}_{4}[\mathrm{M}+\mathrm{H}]^{+}: 249.1293$, found: 249.1292 .

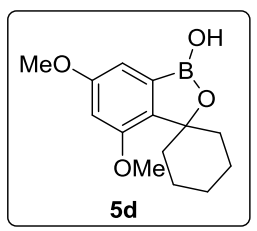

\section{4,6-dimethoxy-1H-spiro[benzo[c] [1,2] oxaborole-3,1'-cyclohexan]-1-ol (5d)}

Conditions: 3,5-dimethoxyphenylboronic acid (91 mg, $0.5 \mathrm{mmol}$ ), cyclohexanone (59 mg, $62 \mu \mathrm{L}$, $0.6 \mathrm{mmol}), \mathrm{TfOH}\left(26 \mathrm{mg}, 15 \mu \mathrm{L}, 0.35\right.$ equiv.), $\mathrm{CHCl}_{3}(5 \mathrm{~mL}), \mathrm{rt}, 24 \mathrm{~h}$. The title product was obtained as white solid (123 mg, 94\%). m.p. $170.7-173.2{ }^{\circ} \mathrm{C} . R_{\mathrm{f}}=0.45$ (PE/EA, 2/1).

${ }^{1} \mathrm{H}$ NMR (400 MHz, DMSO- $\left.d 6\right): \delta 8.95(\mathrm{~s}, 1 \mathrm{H}), 6.77(\mathrm{~d}, J=1.9 \mathrm{~Hz}, 1 \mathrm{H}), 6.60(\mathrm{~d}, J=1.9 \mathrm{~Hz}, 1 \mathrm{H})$, $3.79(\mathrm{~s}, 3 \mathrm{H}), 3.76(\mathrm{~s}, 3 \mathrm{H}), 2.20-2.15(\mathrm{~m}, 2 \mathrm{H}), 1.76-1.56(\mathrm{~m}, 5 \mathrm{H}), 1.26-1.23(\mathrm{~m}, 3 \mathrm{H})$.

${ }^{13}$ C NMR (125 MHz, DMSO- $d 6$ ): $\delta$ 160.6, 154.7, 141.6, 105.0, 101.8, 83.7, 55.5, 55.4, 35.2, 25.0, 22.3.

HRMS (ESI, $m / z$ ) calcd for $\mathrm{C}_{14} \mathrm{H}_{20} \mathrm{BO}_{4}[\mathrm{M}+\mathrm{H}]^{+}: 263.1449$, found: 263.1447 . 


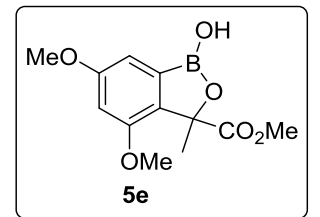

methyl 1-hydroxy-4,6-dimethoxy-3-methyl-1,3-dihydrobenzo[c] [1,2] oxaborole-3carboxylate (5e)

Conditions: 3,5-dimethoxyphenylboronic acid (182 mg, $1 \mathrm{mmol}$ ), methyl pyruvate (122 mg, $108 \mu \mathrm{L}, 1.2 \mathrm{mmol})$, TFA (2280 mg, $1.5 \mathrm{~mL}, 20.0$ equiv.), $\mathrm{CHCl}_{3}(10 \mathrm{~mL}), \mathrm{rt}, 72 \mathrm{~h}$. The title product was obtained as white solid (149 mg, 56\%). m.p.139.6-141.1 ${ }^{\circ} \mathrm{C} . R_{\mathrm{f}}=0.3$ (PE/EA, 2/1).

${ }^{1} \mathrm{H}$ NMR (500 MHz, DMSO-d6): $\delta 9.56(\mathrm{~s}, 1 \mathrm{H}), 6.83(\mathrm{~d}, J=1.8 \mathrm{~Hz}, 1 \mathrm{H}), 6.65(\mathrm{~d}, J=1.8 \mathrm{~Hz}, 1 \mathrm{H}), 3.79(\mathrm{~s}, 3 \mathrm{H}), 3.76(\mathrm{~s}$, $3 \mathrm{H}), 3.56(\mathrm{~s}, 3 \mathrm{H}), 1.63(\mathrm{~s}, 3 \mathrm{H})$.

${ }^{13}$ C NMR (125 MHz, DMSO- $d 6$ ): $\delta$ 171.5, 161.5, 154.7, 136.4, 105.3, 102.1, 83.4, 55.7, 55.5, 52.4, 23.0.

HRMS (ESI, $m / z$ ): calcd for $\mathrm{C}_{12} \mathrm{H}_{16} \mathrm{BO}_{6}[\mathrm{M}+\mathrm{H}]^{+}: 267.1034$, found: 267.1039 .

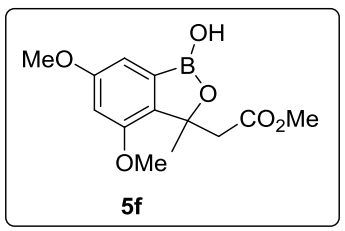

methyl 1-hydroxy-4,6-dimethoxy-3-methyl-1,3-dihydrobenzo[c] [1,2] oxaborole-3carboxylate (5f)

Conditions: 3,5-dimethoxyphenylboronic acid (182 mg, $1 \mathrm{mmol})$, methyl acetoacetate (139 mg ,130 $\mu \mathrm{L}, 1.2 \mathrm{mmol})$, TfOH (150 mg, $88 \mu \mathrm{L}, 1$ equiv.), $\mathrm{CHCl}_{3}(10 \mathrm{~mL}), \mathrm{rt}, 36 \mathrm{~h}$. The title product was obtained as white solid (260 mg, 93\%). m.p. $104.1-106.4{ }^{\circ} \mathrm{C} . R_{\mathrm{f}}=$ 0.40 (PE/EA, 5/1). When the reaction was carried out in $5 \mathrm{mmol}$ scale, $1150 \mathrm{mg}(82 \%)$ of $\mathbf{5 f}$ was obtained.

${ }^{1} \mathrm{H}$ NMR (400 MHz, DMSO-d6): $\delta 9.07$ (s, 1H), 6.75 (d, $\left.J=2.0 \mathrm{~Hz}, 1 \mathrm{H}\right), 6.61$ (d, $\left.J=2.0 \mathrm{~Hz}, 1 \mathrm{H}\right), 3.80$ (s, 3H), 3.77 (s, $3 \mathrm{H}), 3.42(\mathrm{~s}, 3 \mathrm{H}), 2.91$ (d, $J=14.2 \mathrm{~Hz}, 1 \mathrm{H}), 2.76(\mathrm{~d}, J=14.2 \mathrm{~Hz}, 1 \mathrm{H}), 1.51$ (s, 3H).

${ }^{13} \mathrm{C}$ NMR (125 MHz, DMSO- $d 6$ ): $\delta$ 169.9, 161.0, 154.4, 139.1, 104.9, 101.7, 81.9, 55.6, 55.4, 51.0, 43.5, 26.5.

HRMS (ESI, $m / z$ ) calcd for $\mathrm{C}_{13} \mathrm{H}_{18} \mathrm{BO}_{6}[\mathrm{M}+\mathrm{H}]^{+}: 281.1191$, found: 281.1195 .

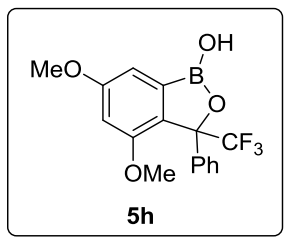

\section{4,6-dimethoxy-3-phenyl-3-(trifluoromethyl) benzo[c] [1,2] oxaborol-1(3H)-ol (5h)}

Conditions: 3,5-dimethoxyphenylboronic acid (182 mg, $1 \mathrm{mmol}$ ), trifluoroacetophenone (209 $\mathrm{mg}, 168 \mu \mathrm{L}, 1.2 \mathrm{mmol})$, TfOH (180 mg, $106 \mu \mathrm{L}, 1.2$ equiv.), $\mathrm{CHCl}_{3}(10 \mathrm{~mL}), \mathrm{rt}, 22 \mathrm{~h}$. The title product was obtained as white solid $(312 \mathrm{mg}, 92 \%)$. m.p. $135.6-138.7{ }^{\circ} \mathrm{C} . R_{\mathrm{f}}=0.40(\mathrm{PE} / \mathrm{EA}$, $5 / 1)$.

${ }^{1} \mathrm{H}$ NMR (400 MHz, DMSO- $\left.d 6\right)$ : $\delta 10.03$ (s, 1H), 7.59-7.55 (m, 2H), 7.41-7.35 (m, 3H), 6.94 (d, $\left.J=1.9 \mathrm{~Hz}, 1 \mathrm{H}\right), 6.75(\mathrm{~d}$, $J=2.1 \mathrm{~Hz}, 1 \mathrm{H}), 3.82(\mathrm{~s}, 3 \mathrm{H}), 3.74(\mathrm{~s}, 3 \mathrm{H})$.

${ }^{13}$ C NMR (125 MHz, DMSO-d6): $\delta$ 162.6, 155.9, 136.1, 131.0, 128.8, 128.3, 127.0, $125.2\left(\mathrm{q}, \mathrm{C}-\mathrm{F},{ }^{1} J \mathrm{C}-\mathrm{F}=283.6 \mathrm{~Hz}\right)$, $106.3,103.2,86.7$ (q, C-F, $\left.{ }^{2} J \mathrm{C}-\mathrm{F}=30.3 \mathrm{~Hz}\right), 55.8,55.6$.

HRMS (ESI, $m / z$ ) calcd for $\mathrm{C}_{16} \mathrm{H}_{15} \mathrm{~B}_{3} \mathrm{FO}_{4}[\mathrm{M}+\mathrm{H}]^{+}: 339.1010$, found: 339.1003 .

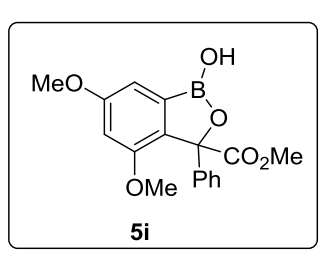

methyl

1-hydroxy-4,6-dimethoxy-3-phenyl-1,3-dihydrobenzo[c]

$[1,2]$ oxaborole-3-carboxylate (5i)

Conditions: 3,5-dimethoxyphenylboronic acid (182 mg, $1 \mathrm{mmol})$, phenyl glyoxylic acid methyl ester (98 mg, $85 \mu \mathrm{L}, 1.2 \mathrm{mmol}$ ), TfOH (38 mg, $22 \mu \mathrm{L}, 0.5$ equiv.), $\mathrm{CHCl}_{3}(10 \mathrm{~mL}$ ), rt, $12 \mathrm{~h}$. The title product was obtained as white solid (282 mg, 86\%). m.p. $141.0-144.0{ }^{\circ} \mathrm{C}$.

$R_{\mathrm{f}}=0.31(\mathrm{PE} / \mathrm{EA}, 2 / 1)$.

${ }^{1} \mathrm{H}$ NMR (400 MHz, DMSO-d6): $\delta 9.66$ (s, 1H), 7.34-7.27 (m, 5H), 6.89 (d, $\left.J=1.9 \mathrm{~Hz}, 1 \mathrm{H}\right), 6.71$ (d, $\left.J=1.9 \mathrm{~Hz}, 1 \mathrm{H}\right)$, $3.81(\mathrm{~s}, 3 \mathrm{H}), 3.72(\mathrm{~s}, 3 \mathrm{H}), 3.63(\mathrm{~s}, 3 \mathrm{H})$.

${ }^{13}$ C NMR (125 MHz, DMSO-d6): $\delta$ 170.7, 161.9, 155.3, 139.4, 134.9, 127.8, 127.7, 127.3, 105.7, 102.7, 87.8, 55.7, 55.6, 52.6 .

HRMS (ESI, $m / z$ ) calcd for $\mathrm{C}_{17} \mathrm{H}_{18} \mathrm{BO}_{6}[\mathrm{M}+\mathrm{H}]^{+}: 329.1191$, found: 329.1190 . 


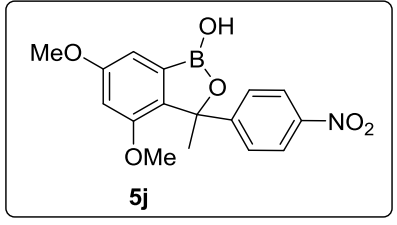

4,6-dimethoxy-3-methyl-3-(4-nitrophenyl) benzo[c] [1,2] oxaborol-1(3H)-ol (5j)

Conditions: 3,5-dimethoxyphenylboronic acid $(182 \mathrm{mg}, \quad 1 \mathrm{mmol})$, 4-nitroacetophenone (198mg, $1.2 \mathrm{mmol}$ ), TfOH (75 mg, $44 \mu \mathrm{L}, 0.5$ equiv.), $\mathrm{CHCl}_{3}$ $(10 \mathrm{~mL}), \mathrm{rt}, 48 \mathrm{~h}$. The title product was obtained as white solid $(287 \mathrm{mg}, 87 \%)$. m.p. $152.1-155.1{ }^{\circ} \mathrm{C} . R_{\mathrm{f}}=0.4(\mathrm{PE} / \mathrm{EA}, 2 / 1)$.

${ }^{1} \mathrm{H}$ NMR (400 MHz, DMSO-d6): $\delta 9.44(\mathrm{~s}, 1 \mathrm{H}), 8.16(\mathrm{~d}, J=8.9 \mathrm{~Hz}, 2 \mathrm{H}), 7.61(\mathrm{~d}, J=8.9 \mathrm{~Hz}, 2 \mathrm{H}), 6.87(\mathrm{~d}, J=1.9 \mathrm{~Hz}$, $1 \mathrm{H}), 6.62(\mathrm{~d}, J=1.9 \mathrm{~Hz}, 1 \mathrm{H}), 3.78$ (s, 3H), 3.70 (s, 3H), 1.90 (s, 3H).

${ }^{13}$ C NMR (125 MHz, DMSO- $\left.d 6\right): \delta 161.5,154.5,151.5,146.6,139.5,127.3,123.1,105.5,102.3,84.3,55.5,55.5,25.9$.

HRMS (ESI, $m / z$ ) calcd for $\mathrm{C}_{16} \mathrm{H}_{17} \mathrm{BNO}_{6}[\mathrm{M}+\mathrm{H}]^{+}: 330.1143$, found: 330.1144

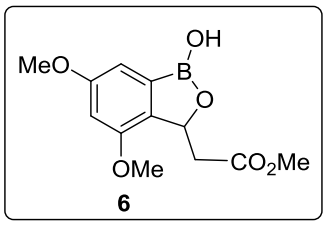

methyl 2-(1-hydroxy-4,6-dimethoxy-1,3-dihydrobenzo[c] [1,2] oxaborol-3-yl) acetate (6)

Conditions: 3,5-dimethoxyphenylboronic acid (182 $\mathrm{mg}, \quad 1 \mathrm{mmol})$, methyl 3,3-dimethoxypropionate ( $222 \mathrm{mg}, 212 \mu \mathrm{L}, 1.5 \mathrm{mmol}$ ), TFA (570 mg, $371 \mu \mathrm{L}, 5$ equiv.), $\mathrm{CHCl}_{3}(10 \mathrm{~mL}), \mathrm{rt}, 60 \mathrm{~h}$. The title product was obtained as white solid (186 mg, 70\%). m.p. $130.0-132.3{ }^{\circ} \mathrm{C} . R_{\mathrm{f}}=0.39(\mathrm{PE} / \mathrm{EA}, 2 / 1)$

${ }^{1} \mathrm{H}$ NMR (400 MHz, DMSO- $\left.d 6\right): \delta 9.23(\mathrm{~s}, 1 \mathrm{H}), 6.80(\mathrm{~d}, J=1.9 \mathrm{~Hz}, 1 \mathrm{H}), 6.64(\mathrm{~d}, J=1.9 \mathrm{~Hz}, 1 \mathrm{H}), 5.38(\mathrm{dd}, J=9.7,2.8$ $\mathrm{Hz}, 1 \mathrm{H}), 3.80$ (s, 3H), 3.78 (s, 3H), 3.63 (s, 3H), 3.15 (dd, $J=15.4,2.9 \mathrm{~Hz}, 1 \mathrm{H}), 2.21$ (dd, $J=15.4,9.7 \mathrm{~Hz}, 1 \mathrm{H})$.

${ }^{13} \mathrm{C}$ NMR (125 MHz, DMSO- $d 6$ ): $\delta$ 171.0, 161.3, 154.6, 135.3, 105.1, 101.6, 75.7, 55.5, 55.5, 51.5, 40.2.

HRMS (ESI, $m / z$ ) calcd for $\mathrm{C}_{12} \mathrm{H}_{16} \mathrm{BO}_{6}[\mathrm{M}+\mathrm{H}]^{+}: 267.1034$, found: 267.1034 .

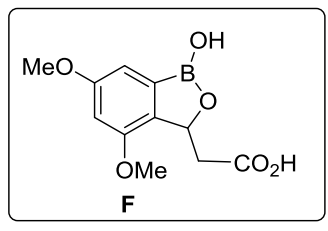

2-(1-hydroxy-4,6-dimethoxy-1,3-dihydrobenzo[c][1,2]oxaborol-3-yl)acetic acid (F) Following a literature procedure, ${ }^{4}$ to a solution of compound $6(133 \mathrm{mg}, 0.5 \mathrm{mmol})$ in methanol $(2.5 \mathrm{~mL})$ was added an aqueous solution of lithium hydroxide $(2 \mathrm{M}, 1.25 \mathrm{~mL})$ at 0 ${ }^{\circ} \mathrm{C}$. The reaction mixture was stirred at $\mathrm{rt}$ overnight and acidified to $\mathrm{pH}=2$ with $1 \mathrm{M} \mathrm{HCl}$. The resulting mixture was extracted with ethyl acetate, and the combined organic layers were dried over anhydrous $\mathrm{Na}_{2} \mathrm{SO}_{4}$ and concentrated in vacuum. The residue was purified by silica gel column chromatography. The title product was obtained as white solid (63 mg, 50\%). The spectra data matched the literature. ${ }^{4} R_{\mathrm{f}}$ $=0.25(\mathrm{EA})$

${ }^{1} \mathrm{H}$ NMR (500 MHz, DMSO-d6): $\delta 12.26(\mathrm{~s}, 1 \mathrm{H}), 9.18(\mathrm{~s}, 1 \mathrm{H}), 6.80(\mathrm{~d}, J=1.9 \mathrm{~Hz}, 1 \mathrm{H}), 6.63(\mathrm{~d}, J=1.9 \mathrm{~Hz}, 1 \mathrm{H}), 5.36(\mathrm{dd}$, $J=9.8,2.6 \mathrm{~Hz}, 1 \mathrm{H}), 3.80(\mathrm{~s}, 3 \mathrm{H}), 3.78(\mathrm{~s}, 3 \mathrm{H}), 3.08(\mathrm{dd}, J=15.4,2.6 \mathrm{~Hz}, 1 \mathrm{H}), 2.06(\mathrm{dd}, J=15.4,9.9 \mathrm{~Hz}, 1 \mathrm{H})$

${ }^{13} \mathrm{C}$ NMR (125 MHz, DMSO- $\left.d 6\right): \delta 172.1,161.2,154.6,135.5,105.0,101.6,75.9,55.5,55.5,40.5$

\section{References}

(1) (a) Ding, D.; Zhao, Y.; Meng, Q.; Xie, D.; Nare, B.; Chen, D.; Bacchi, C. J.; Yarlett, N.; Zhang, Y.-K.; Hernandez, V.; Xia, Y.; Freund, Y.; Abdulla,M.; Ang, K.-H.; Ratnam, J.; McKerrow, J. H.; Jacobs, R. T.; Zhou, H.; Plattner, J. J. ACS Med. Chem. Lett. 2010, 1, 165. (b) Ding, D.; Meng, Q.; Gao, G.; Zhao, Y.; Wang, Q.; Nare, B.; Jacobs, R.; Rock, F.; Alley, M. R. K.; Plattner, J. J.; Chen, G.; Li, D.; Zhou, H. J. Med. Chem. 2011, 54, 1276.

(2) Xia, Y.; Cao, K.; Zhou, Y.; Alley, M. R. K.; Rock, F.; Mohan, M.; Meewan, M.; Baker, S. J.; Lux, S.; Ding, C. Z.; Jia, G.; Kully, M.; Plattner, J. J. Bioorg. Med. Chem. Lett. 2011, 21, 2533.

(3) Shao, A.; Li, N.; Gao, Y.; Zhan, J.; Chiang, C.-W.; Lei, A. Chin. J. Chem. 2018, 36, 619.

(4) Xia, Y.; Alley, M. R. K.; Zhou, Y.; Singh, R.; Ding, C.; Cao, K.; Plattner, J. J.; Ou, L.; Jia, G.; Saraswat, N.; Ramachandran, S.; Zhou, D. PCT Int. Appl. WO 2011/017125 A1, February 10, 2011. 


\section{4. ${ }^{1} \mathrm{H}$ and ${ }^{13} \mathrm{C}$ NMR spectra}

4,6-dimethoxybenzo[c] [1,2] oxaborol-1(3H)-ol (3a)

${ }^{1}$ H NMR (400 MHz, DMSO-d6)

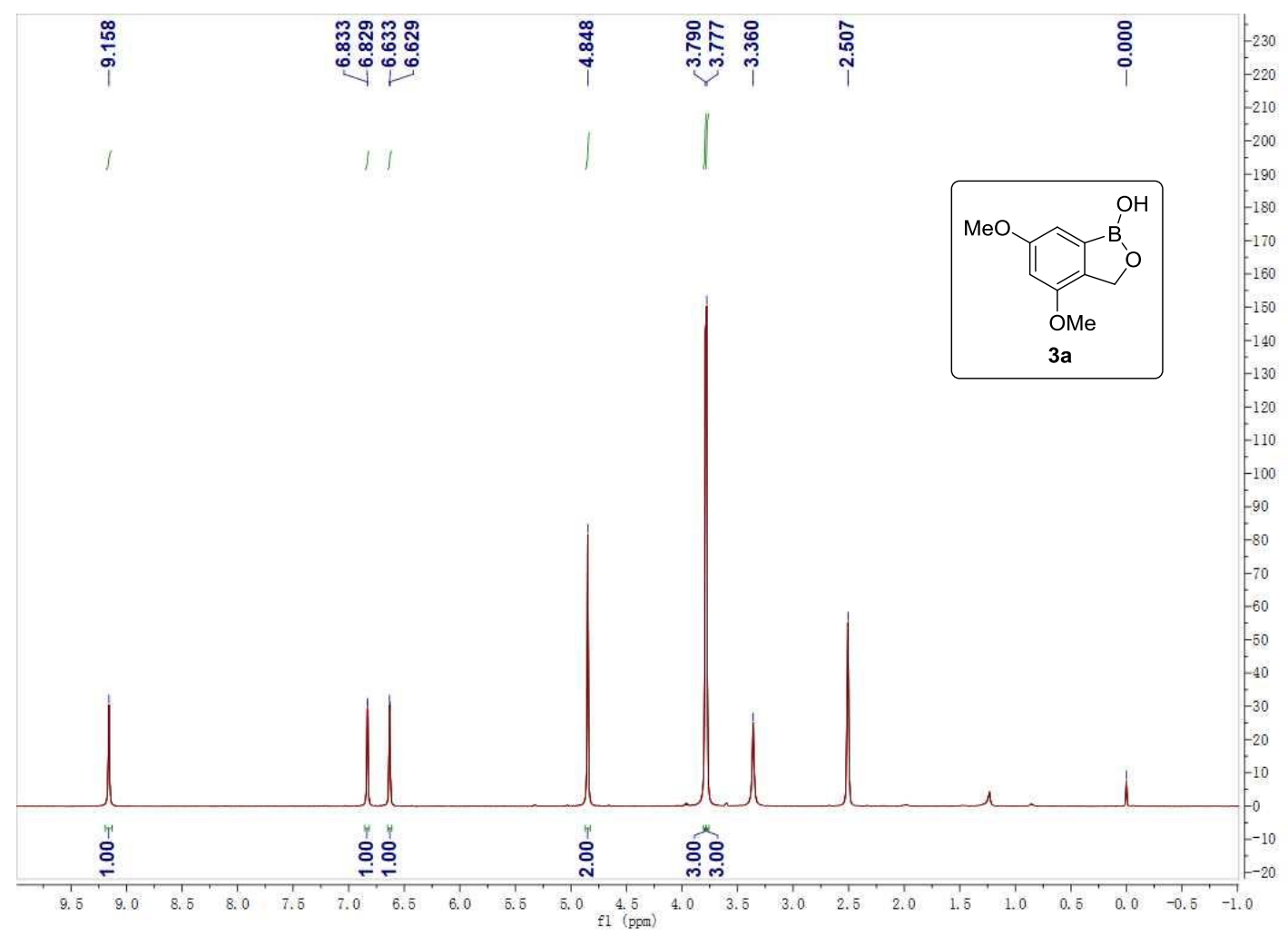

${ }^{13}$ C NMR (125 MHz, DMSO-d6)

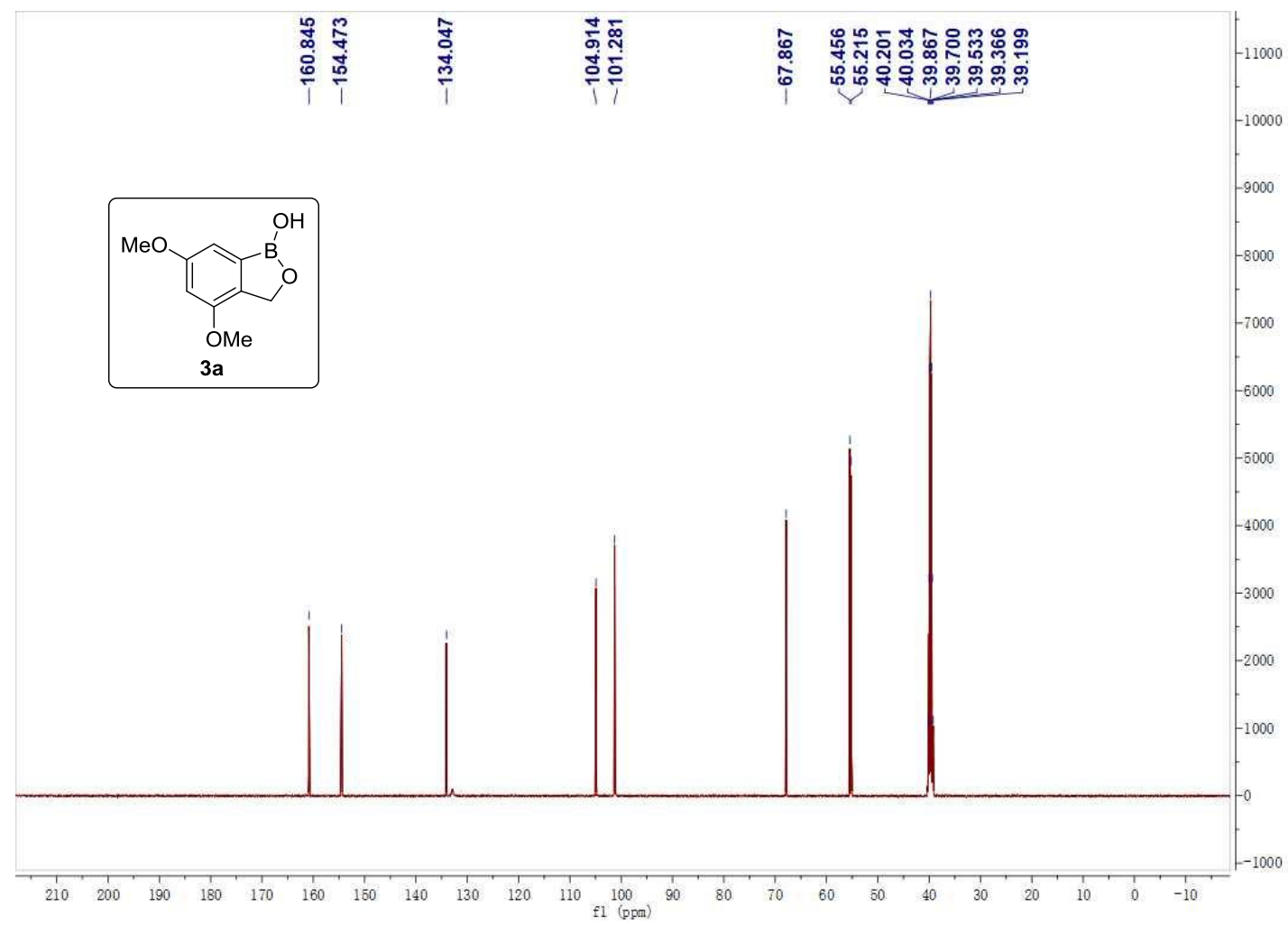


4,5,6-trimethoxybenzo[c] $[1,2]$ oxaborol-1 $(3 \mathrm{H})$-ol (3b)

${ }^{1}$ H NMR (500 MHz, DMSO-d6)

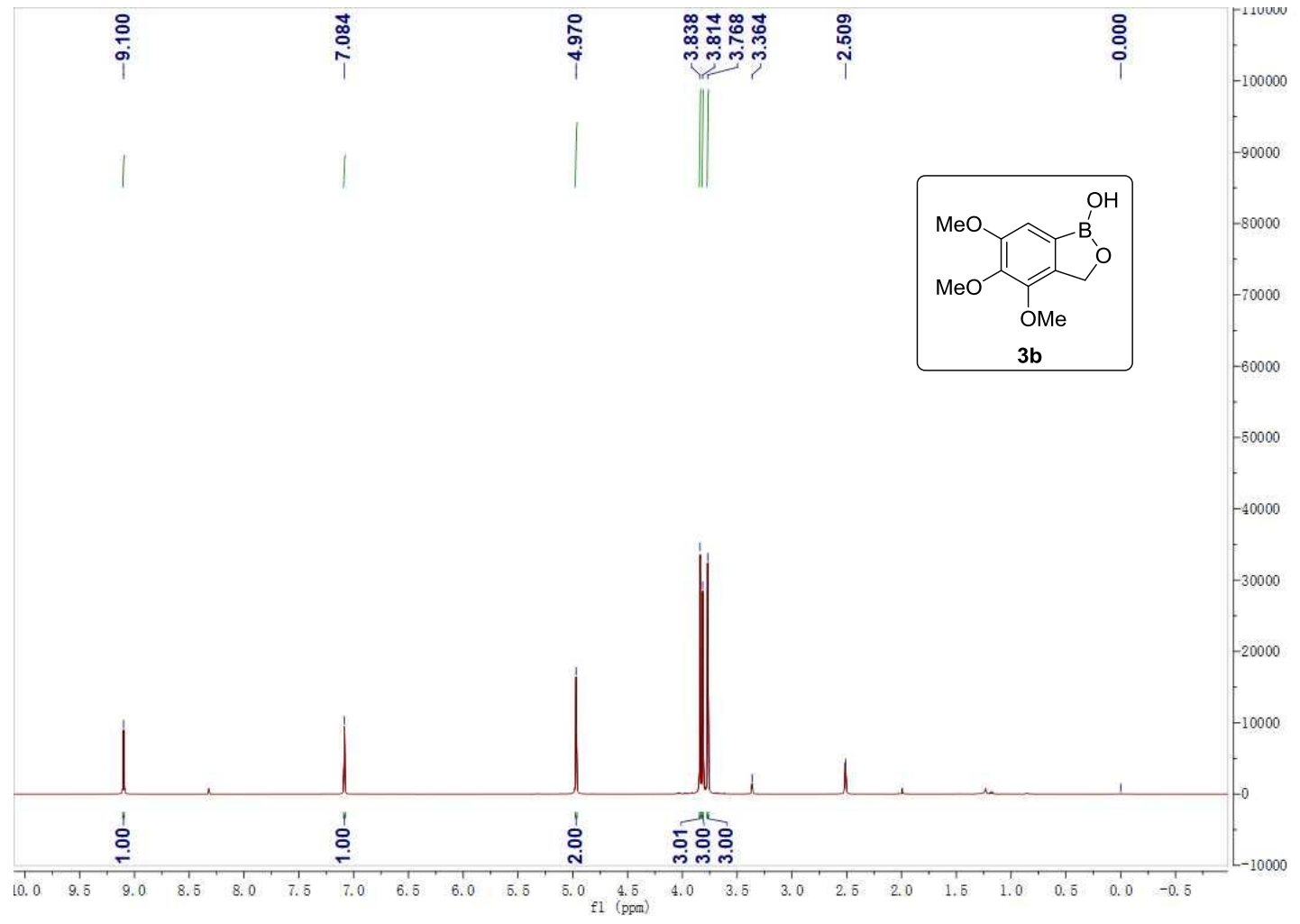

${ }^{13}$ C NMR (125 MHz, DMSO-d6)

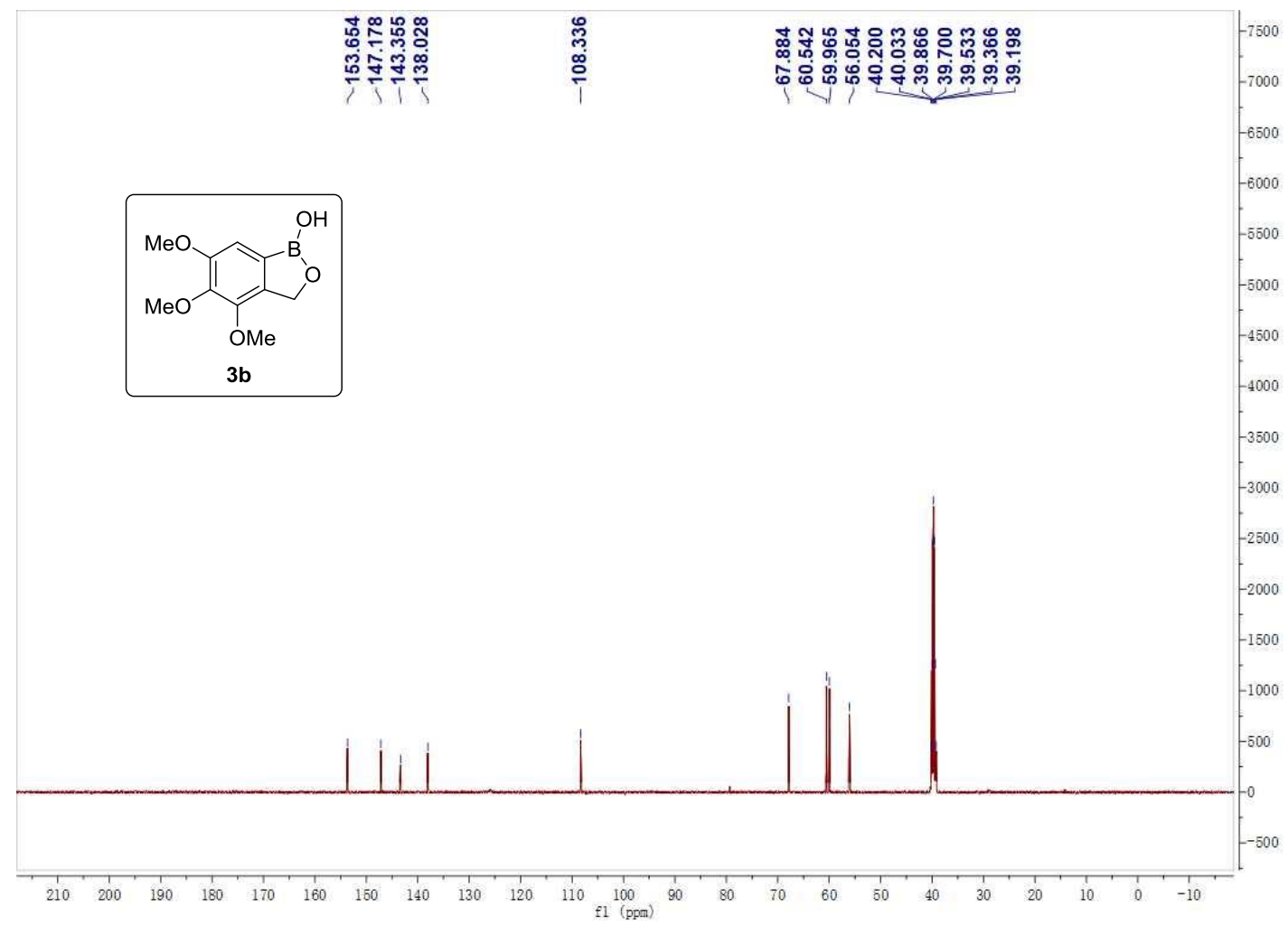


6-methoxy-5-methylbenzo[c] [1,2] oxaborol-1(3H)-ol (3c)

${ }^{1}$ H NMR (400 MHz, DMSO-d6)

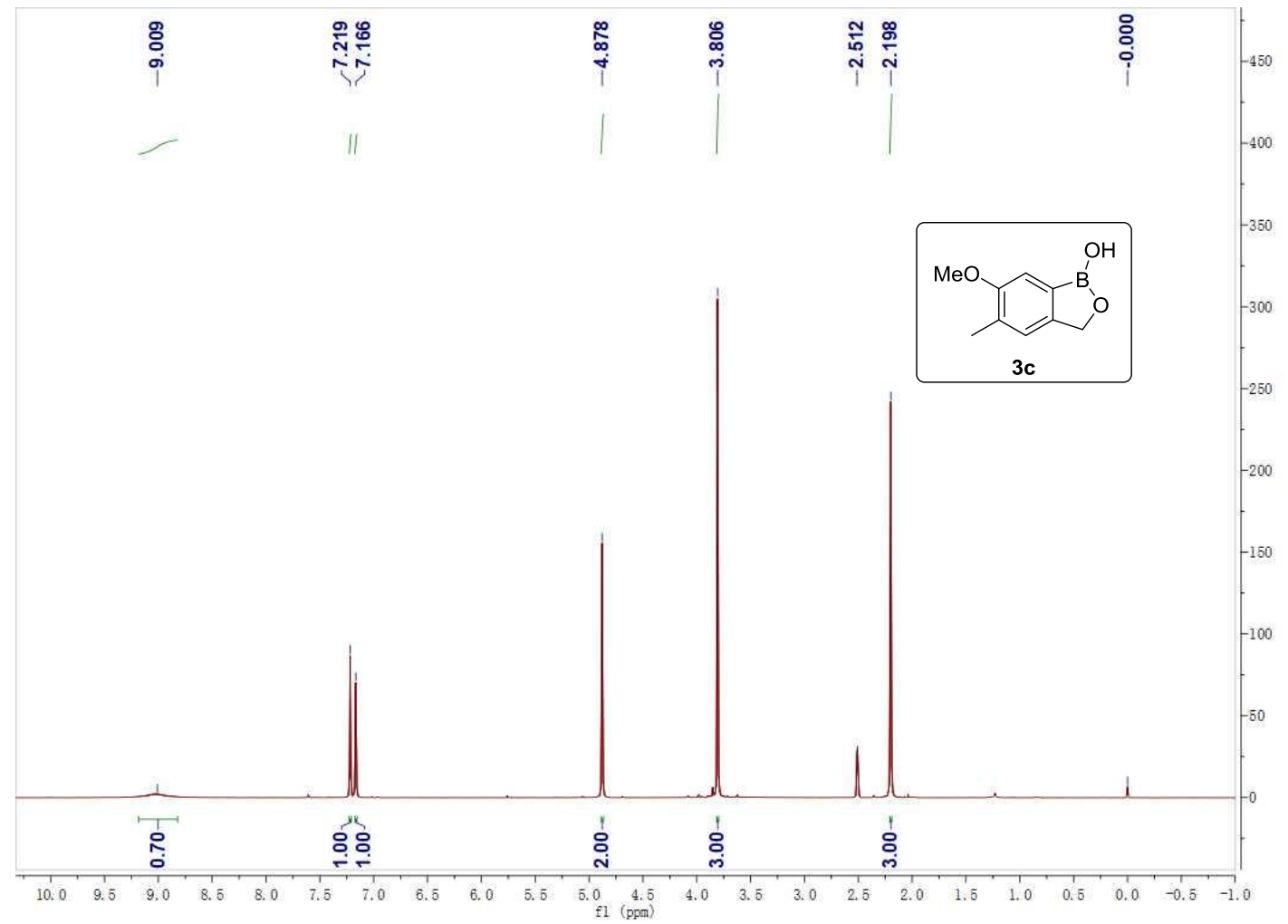

${ }^{13}$ C NMR (125 MHz, DMSO- $d 6$ )

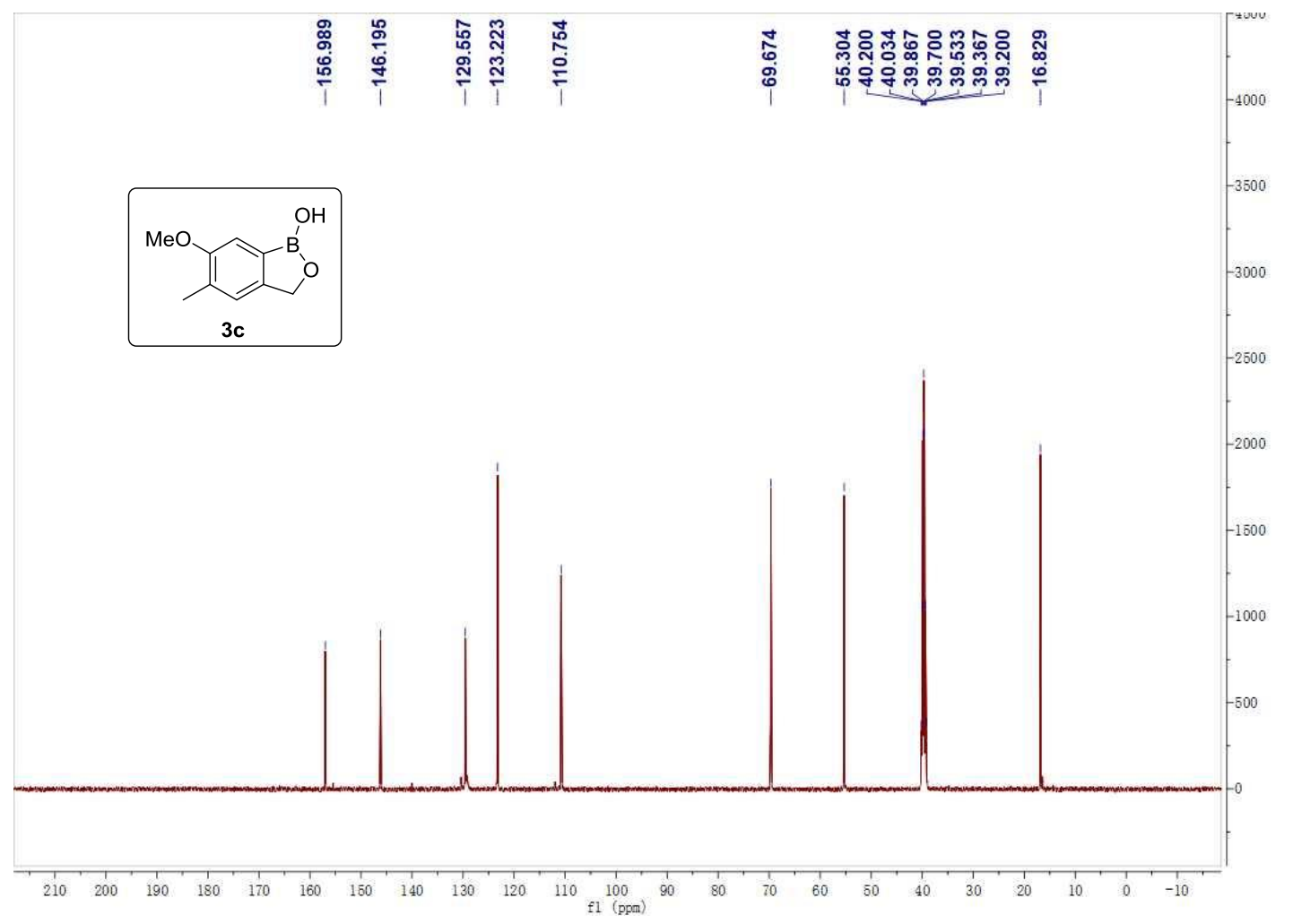


6-(benzyloxy) benzo[c] [1,2] oxaborol-1(3H)-ol (3d)

${ }^{1}$ H NMR (400 MHz, DMSO-d6)

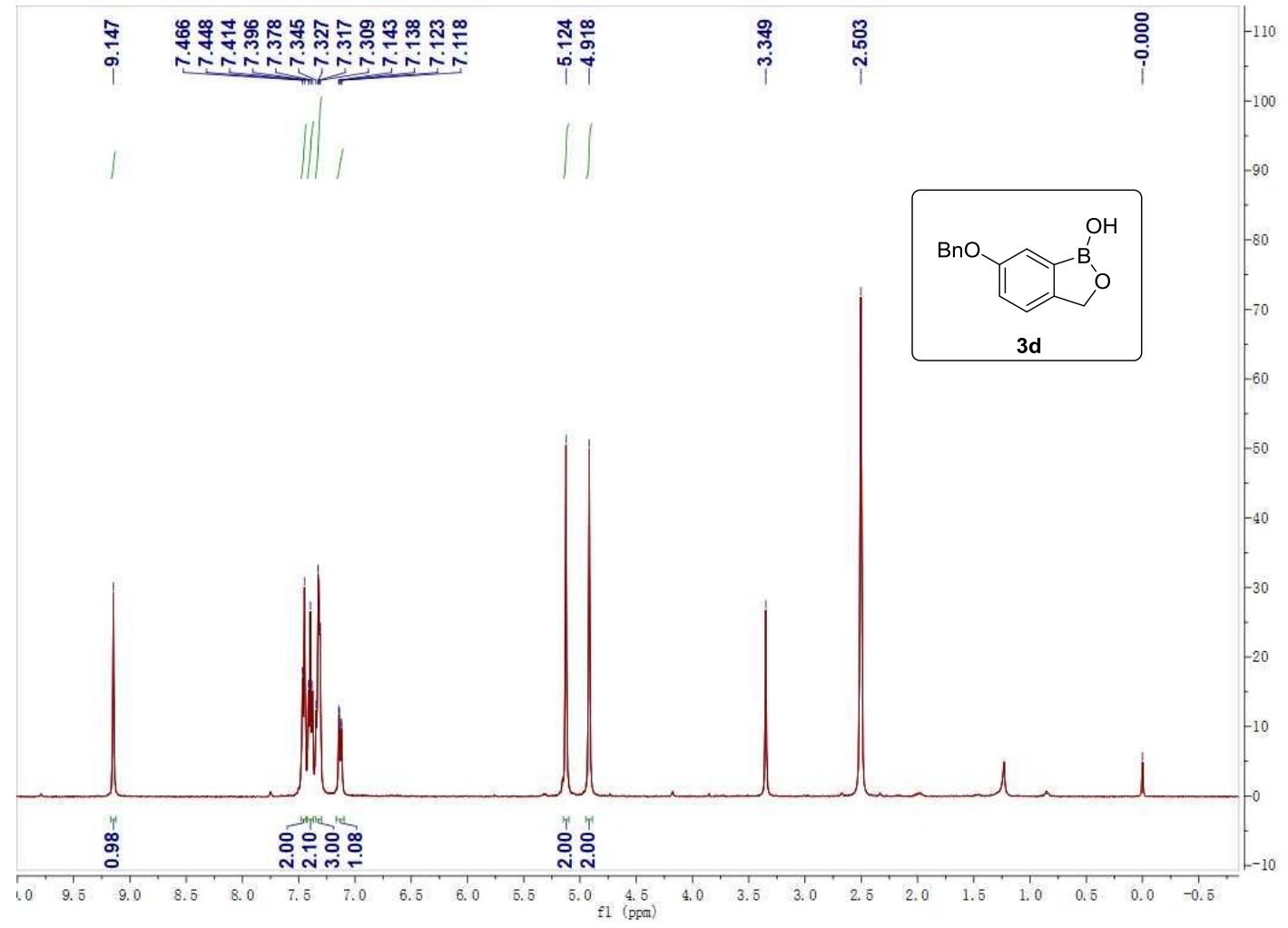

${ }^{13}$ C NMR (125 MHz, DMSO-d6)

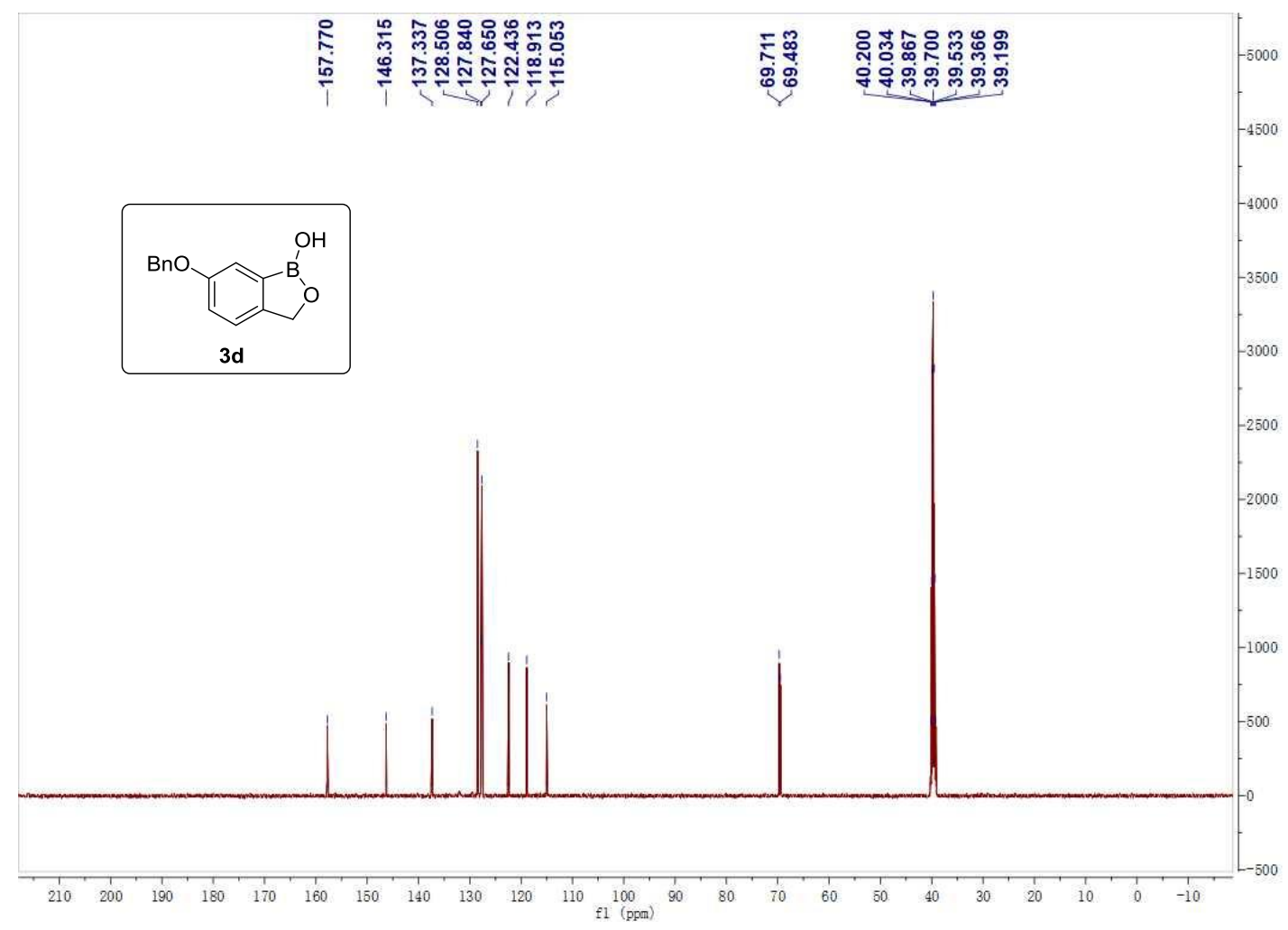


6-methoxybenzo[c] [1,2] oxaborol-1(3H)-ol (3e)

${ }^{\mathbf{1}} \mathbf{H}$ NMR (400 MHz, DMSO-d6)

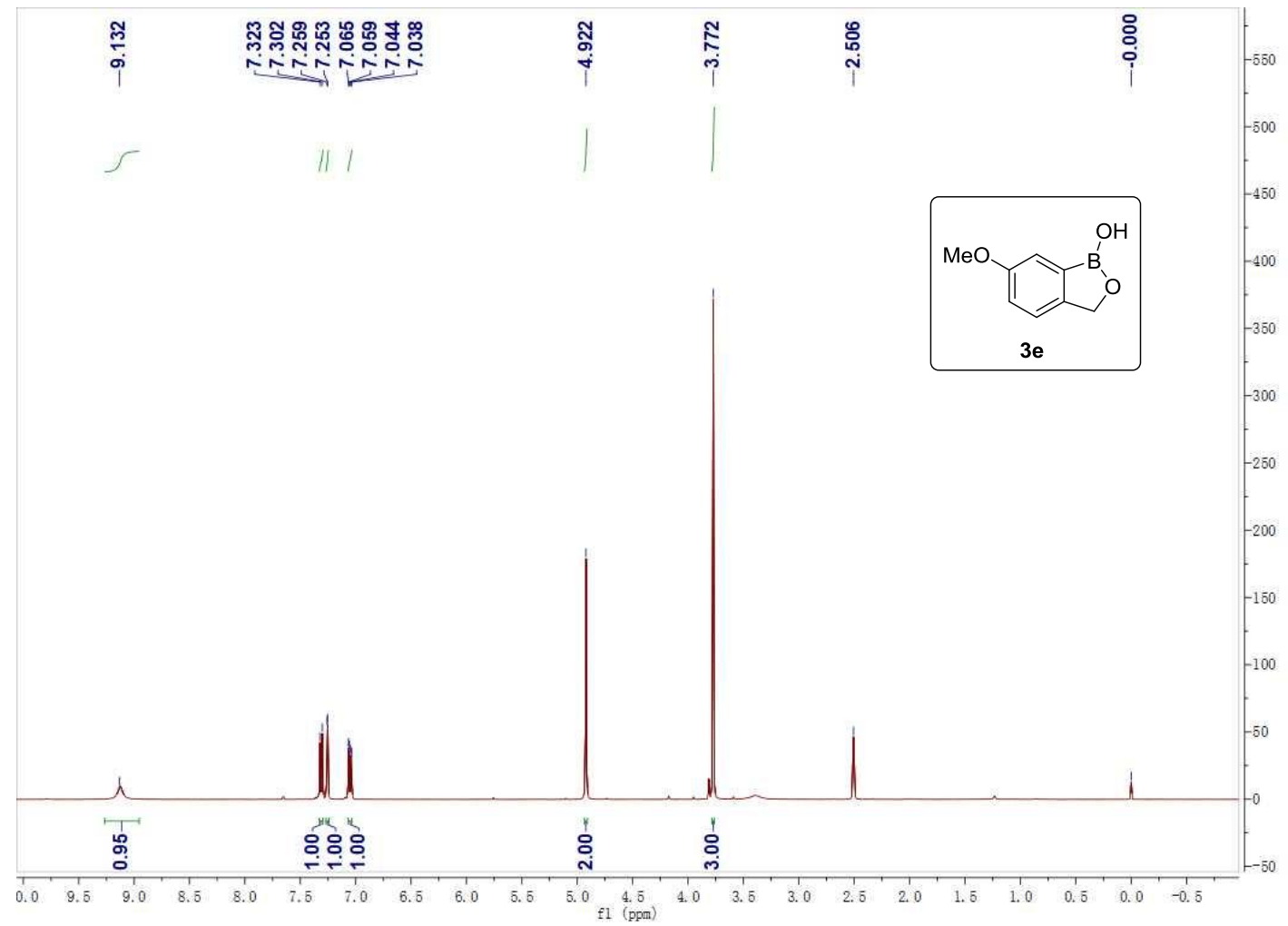

${ }^{13}$ C NMR (125 MHz, DMSO-d6)

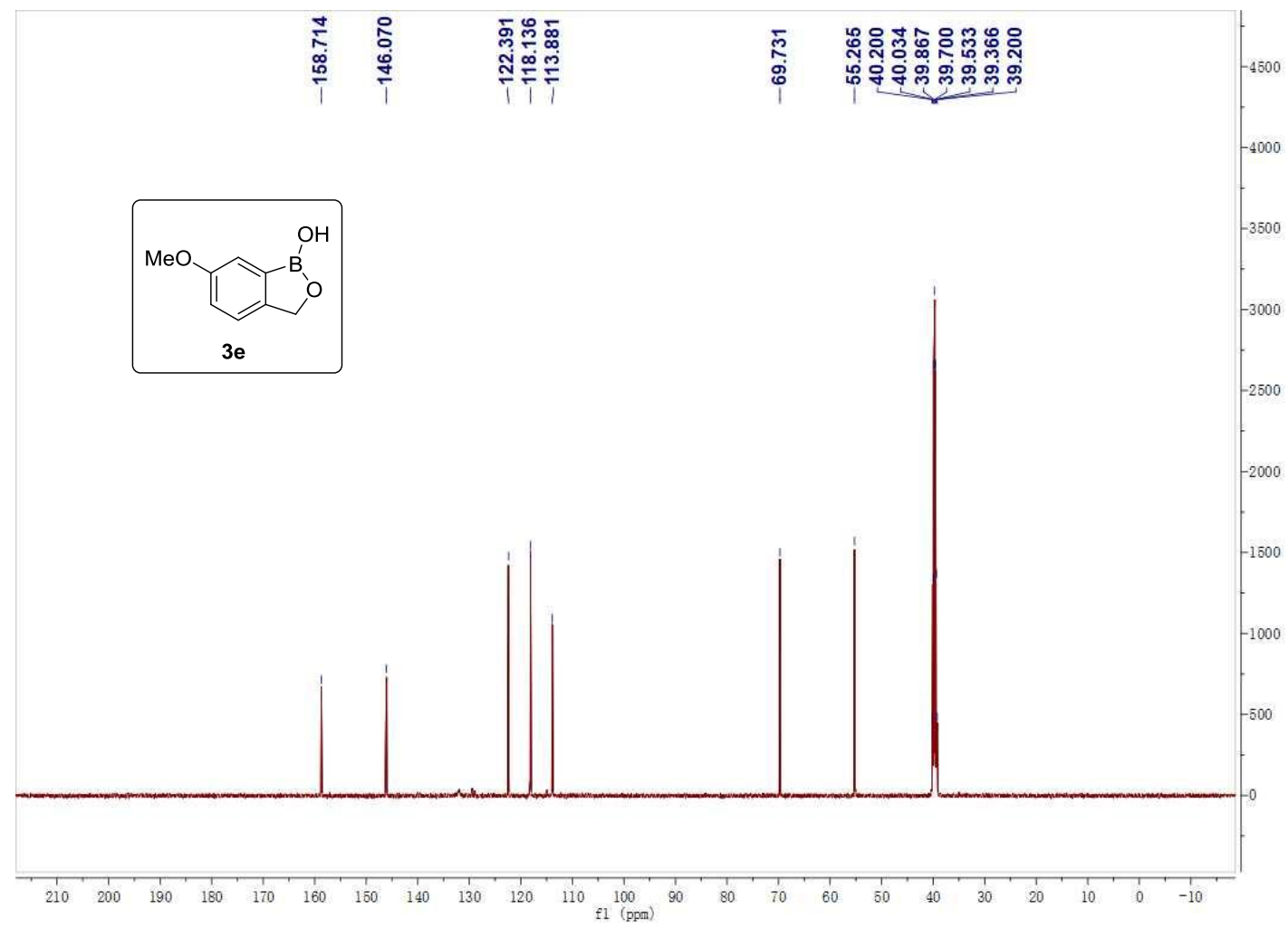


6-methoxy-4-methylbenzo[c] [1,2] oxaborol-1(3H)-ol (3f)

${ }^{1}$ H NMR (400 MHz, DMSO-d6)

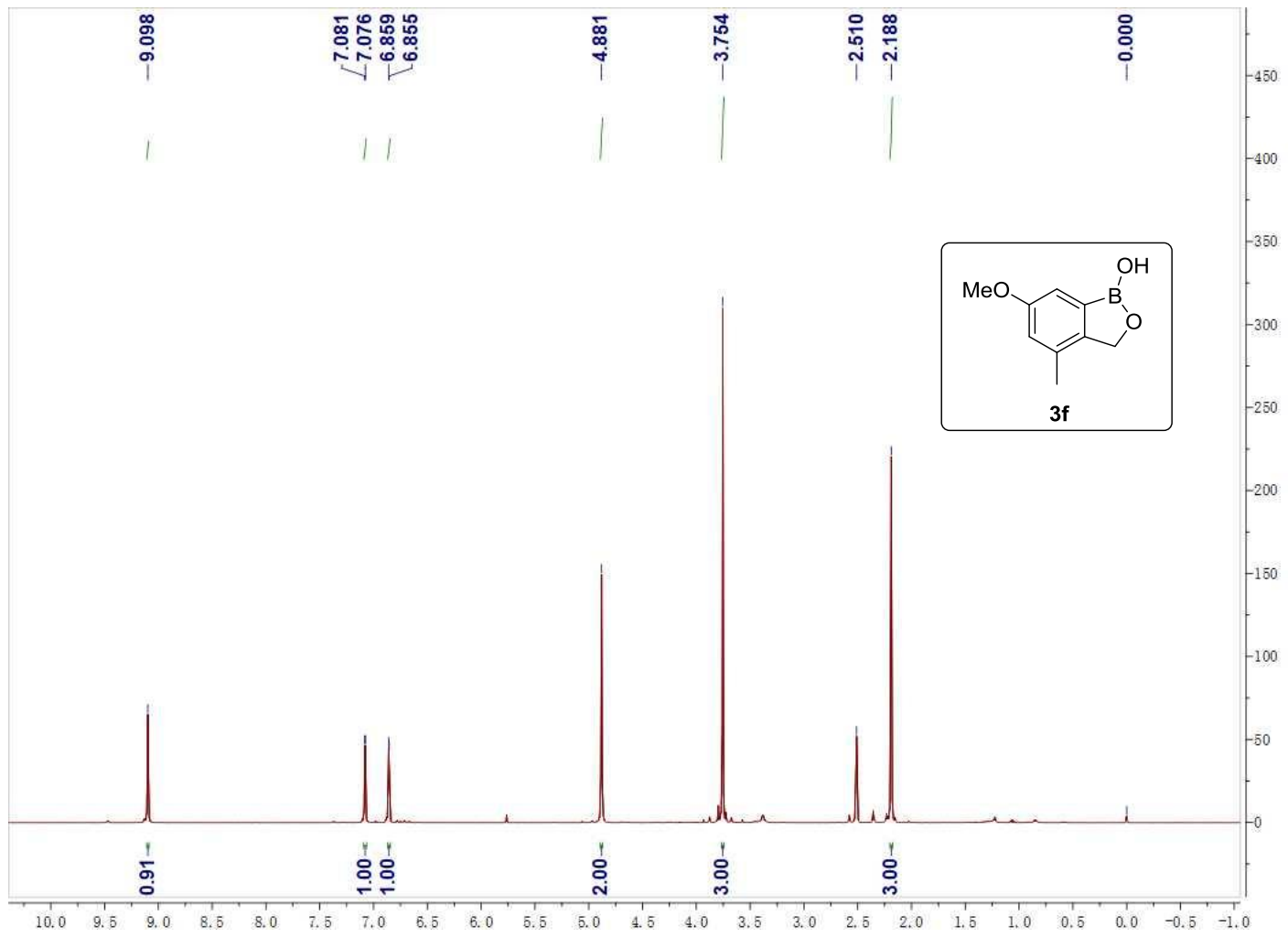

${ }^{13}$ C NMR (125 MHz, DMSO- $d 6$ )

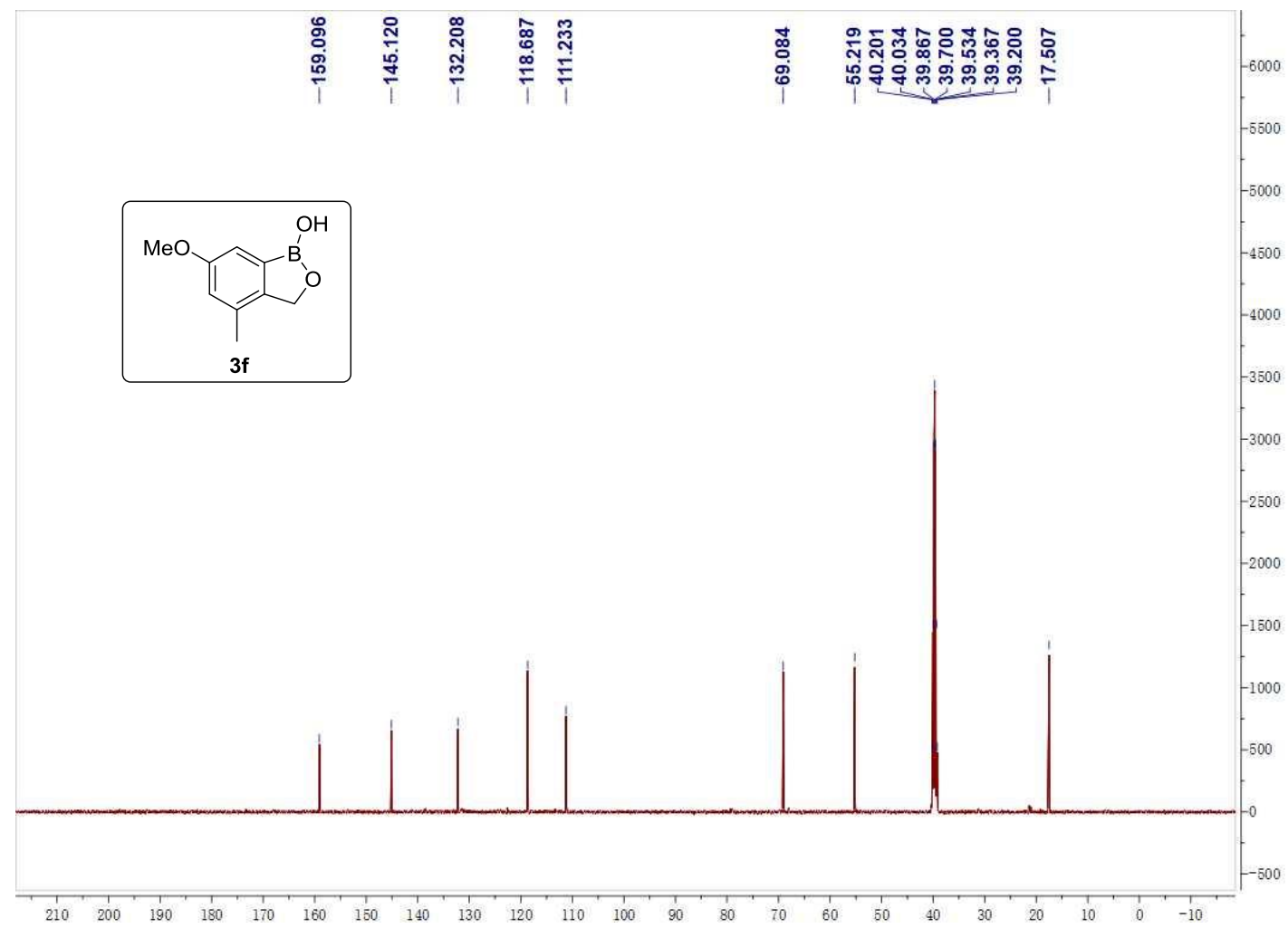


4-fluoro-6-methoxybenzo[c] [1,2] oxaborol-1(3H)-ol (3g)

${ }^{1}$ H NMR (400 MHz, DMSO-d6)

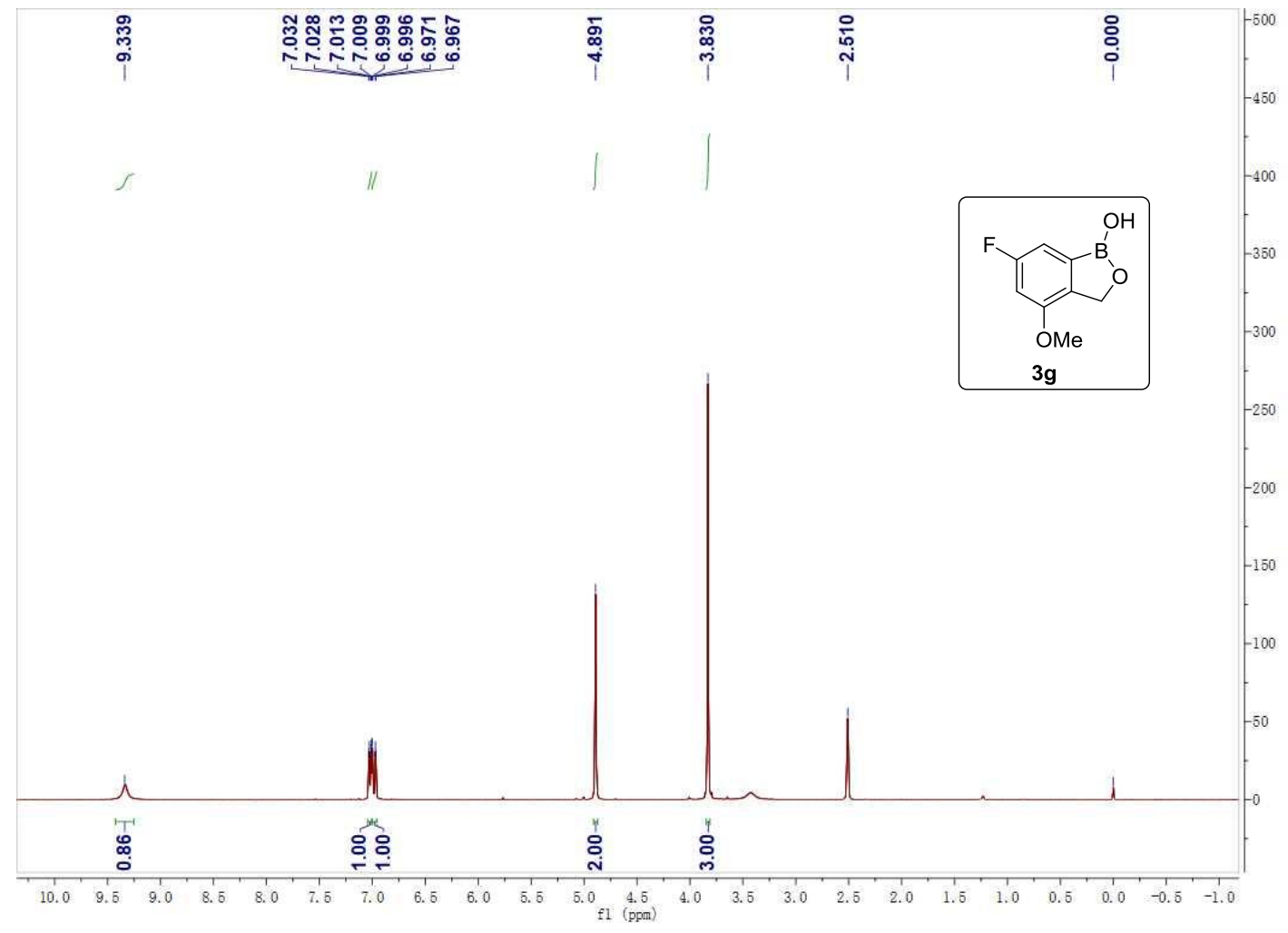

${ }^{13}$ C NMR (125 MHz, DMSO-d6)

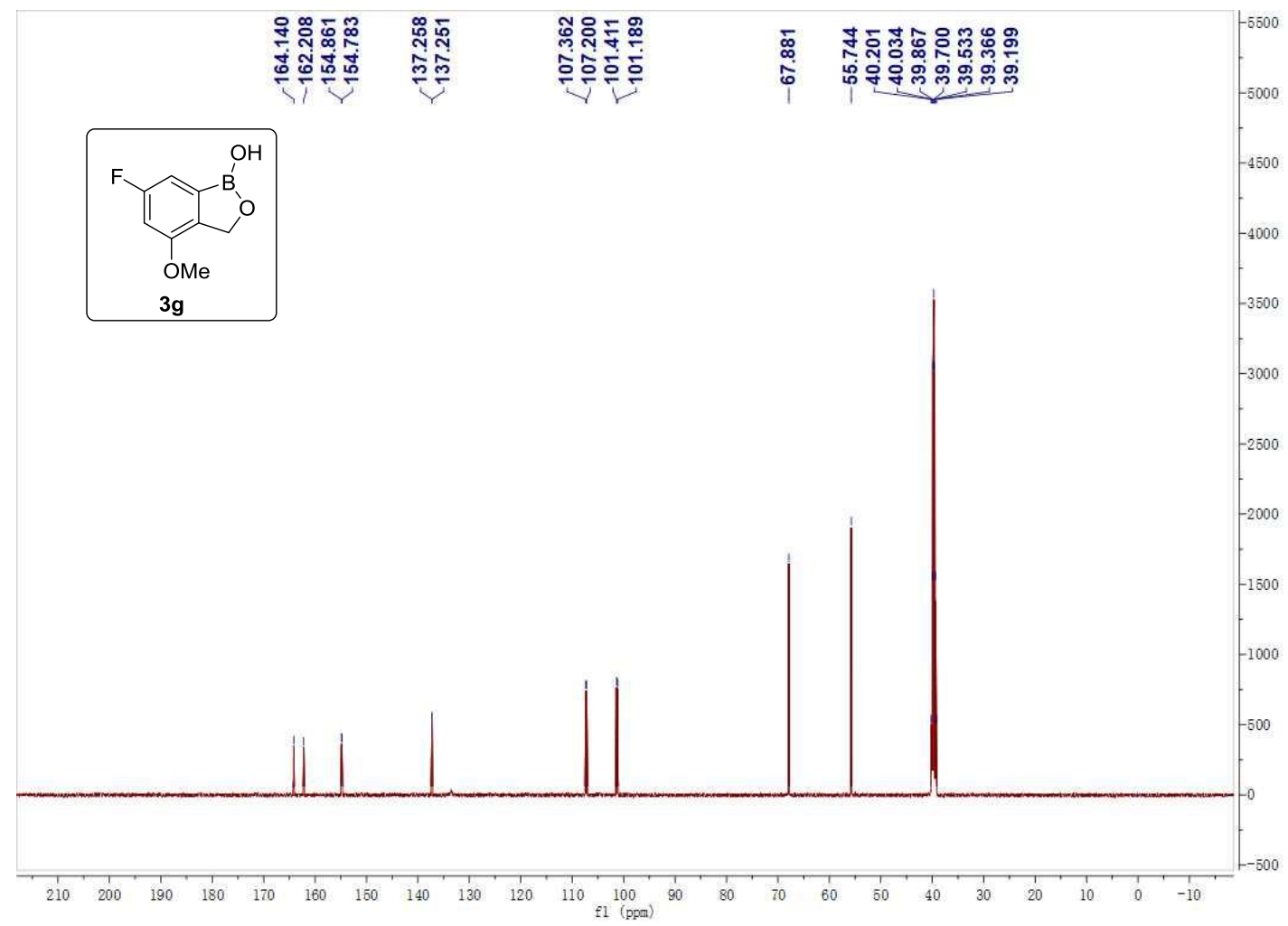


5-fluoro-6-methoxybenzo[c] [1,2] oxaborol-1(3H)-ol (3h)

${ }^{1}$ H NMR (400 MHz, DMSO-d6)

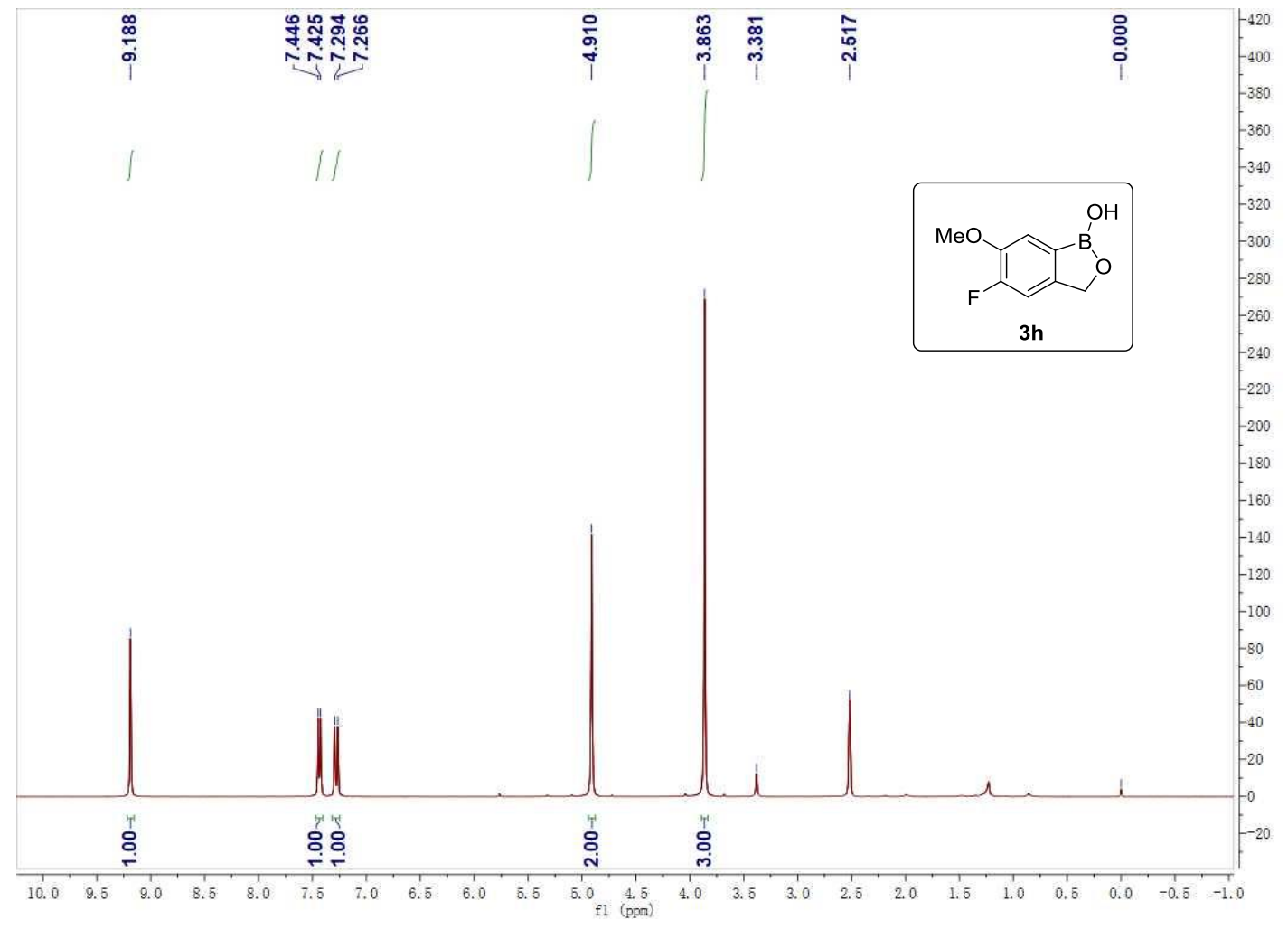

${ }^{13}$ C NMR (125 MHz, DMSO- $d 6$ )

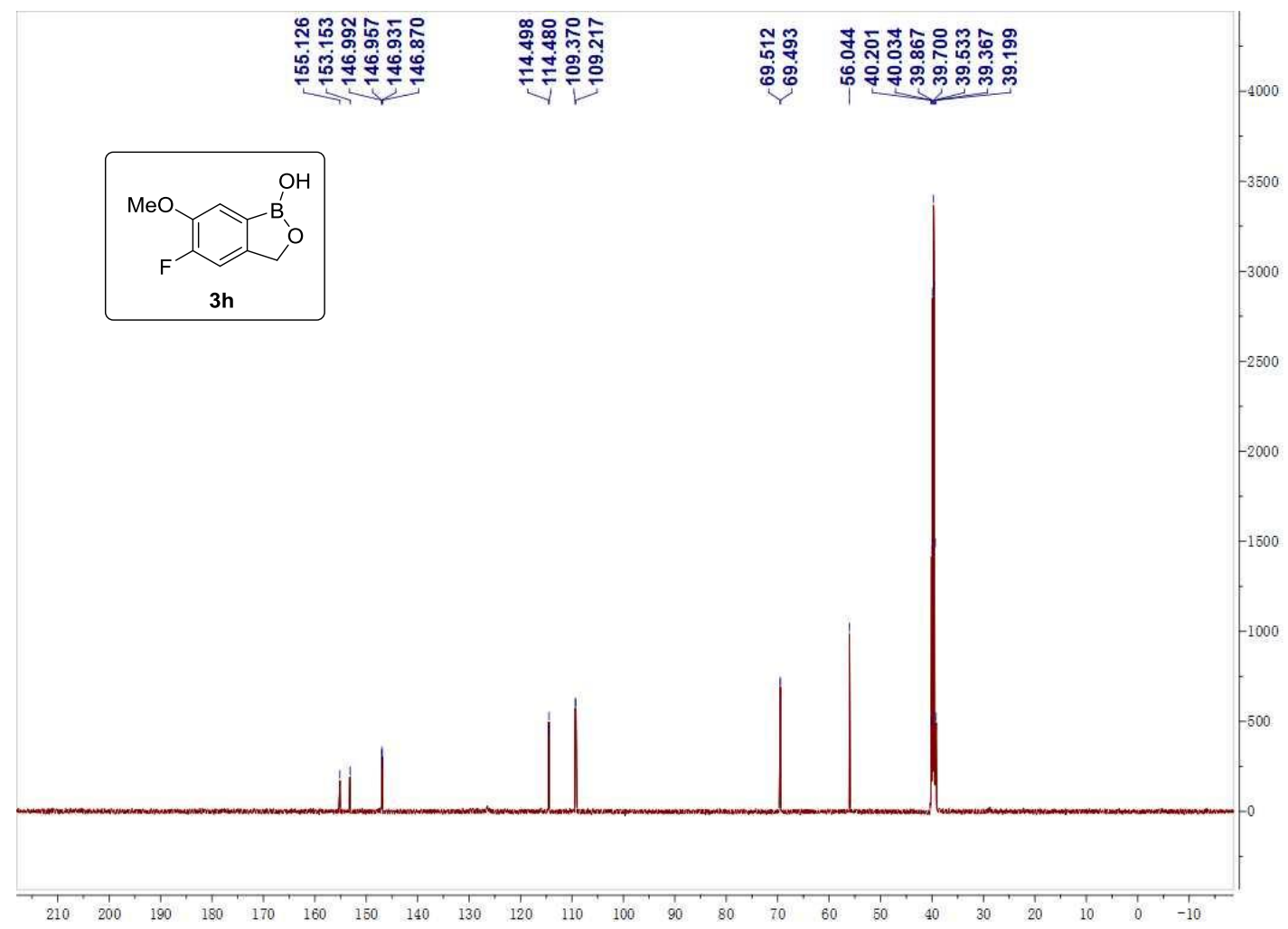


6-methoxybenzo[c] [1,2] oxaborol-1(3H)-ol (3i)

${ }^{1}$ H NMR (500 MHz, DMSO-d6)

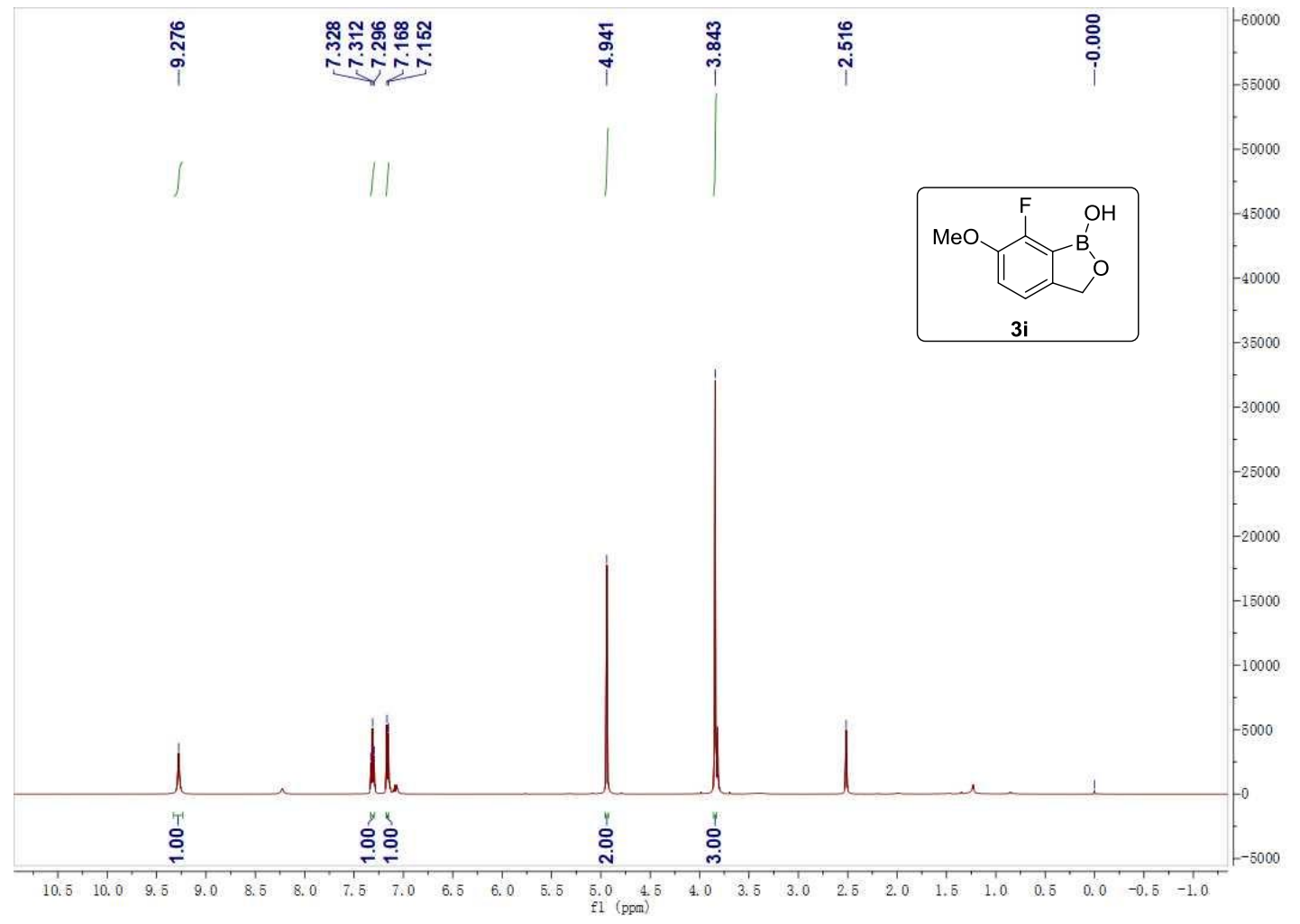

${ }^{13}$ C NMR (125 MHz, DMSO- $d 6$ )

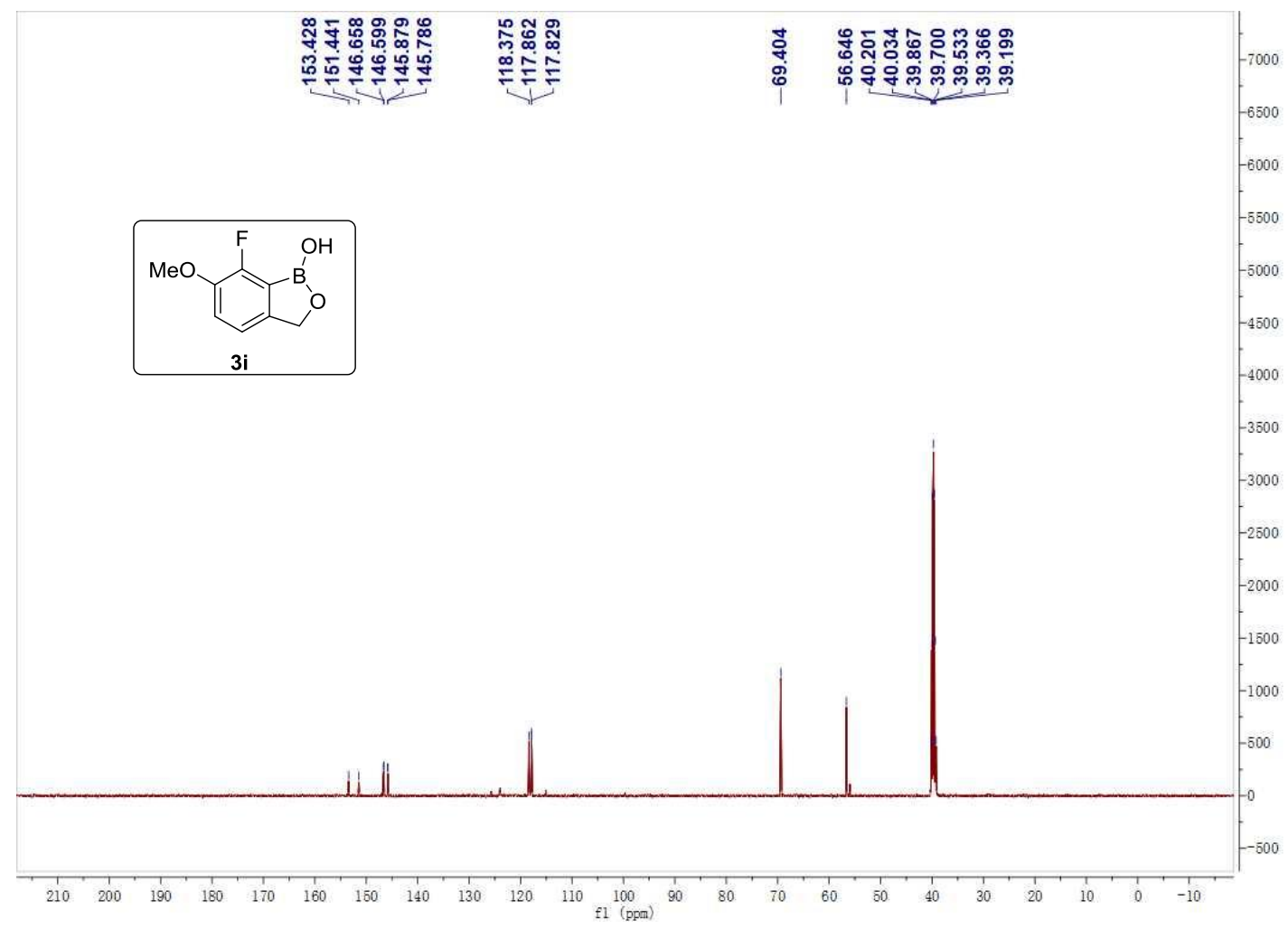


7-fluoro-4-methoxybenzo[c] [1,2] oxaborol-1(3H)-ol (3j)

${ }^{1}$ H NMR (400 MHz, DMSO-d6)

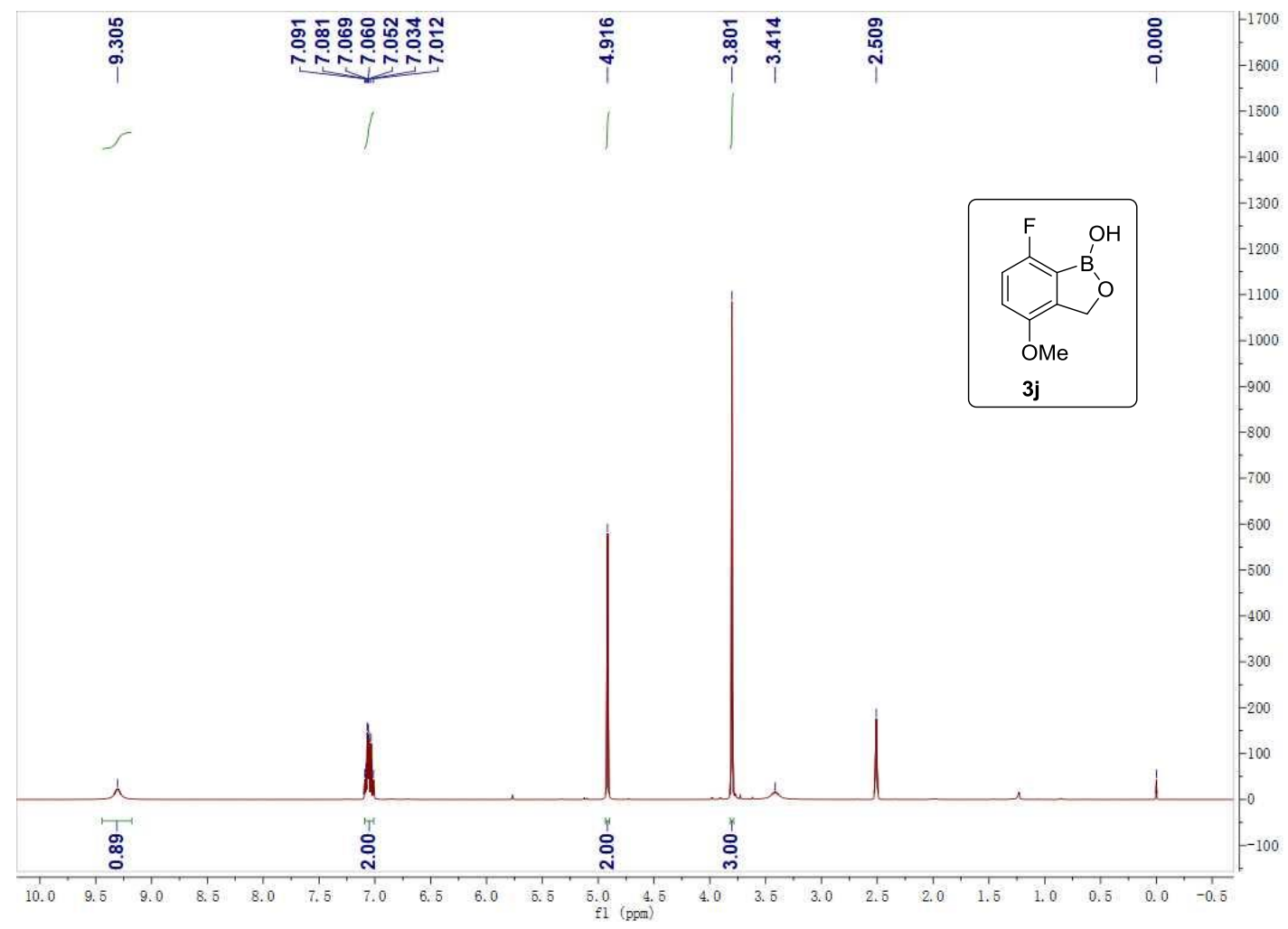

${ }^{13}$ C NMR (125 MHz, DMSO- $d 6$ )

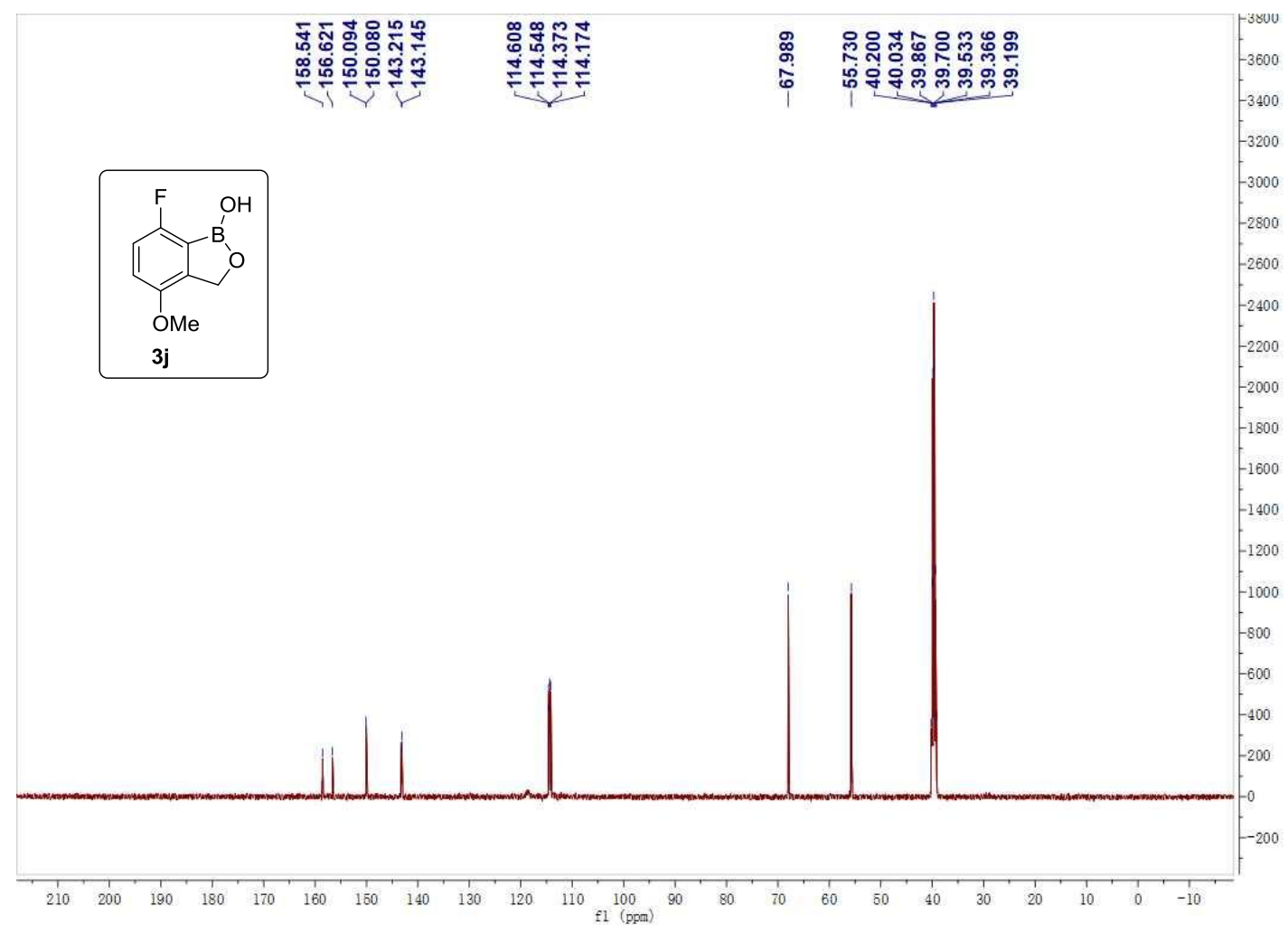


7-chloro-4-methoxybenzo[c] [1,2] oxaborol-1(3H)-ol (3k)

${ }^{1}$ H NMR (400 MHz, DMSO-d6)

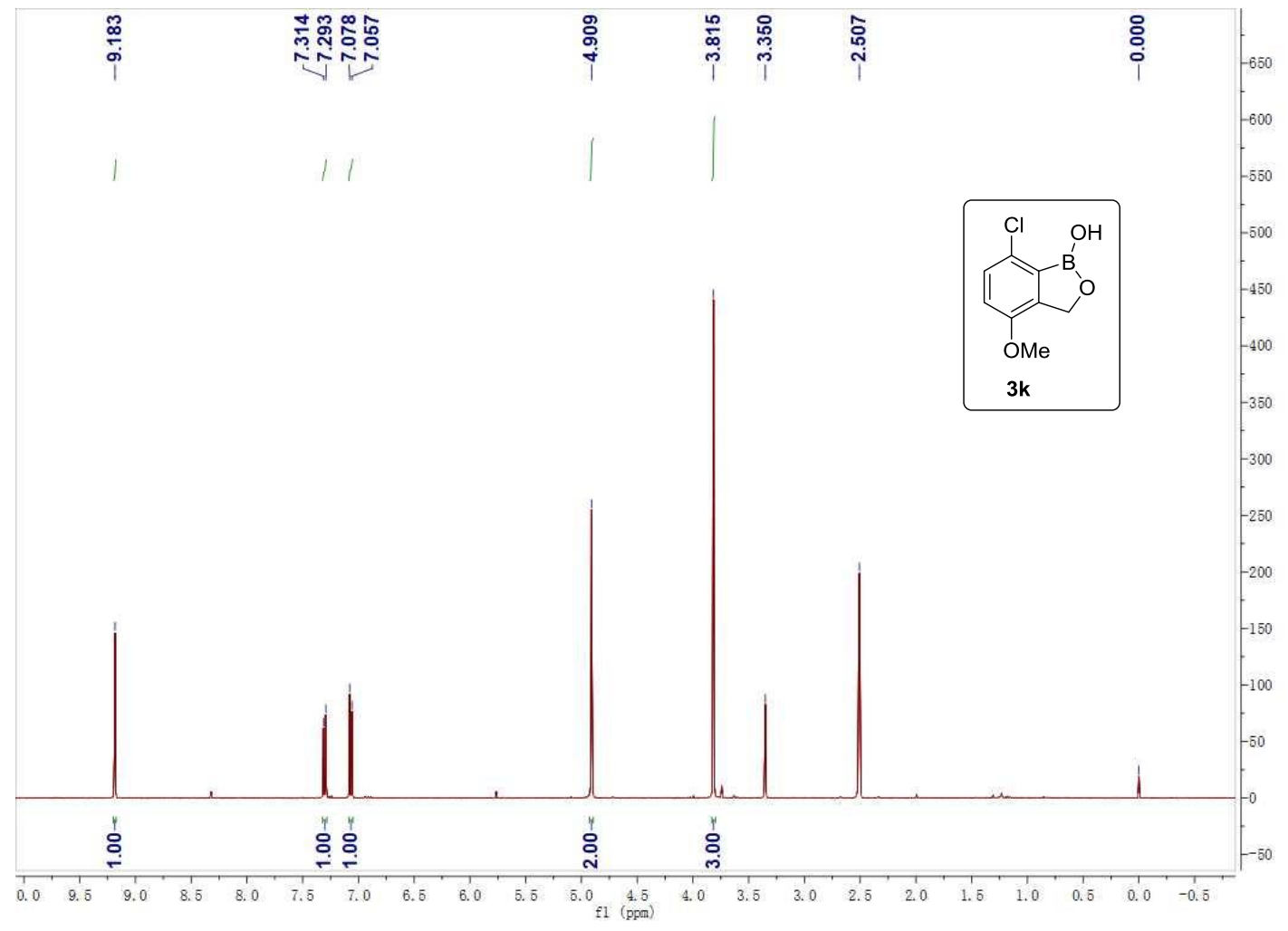

${ }^{13}$ C NMR (125 MHz, DMSO-d6)

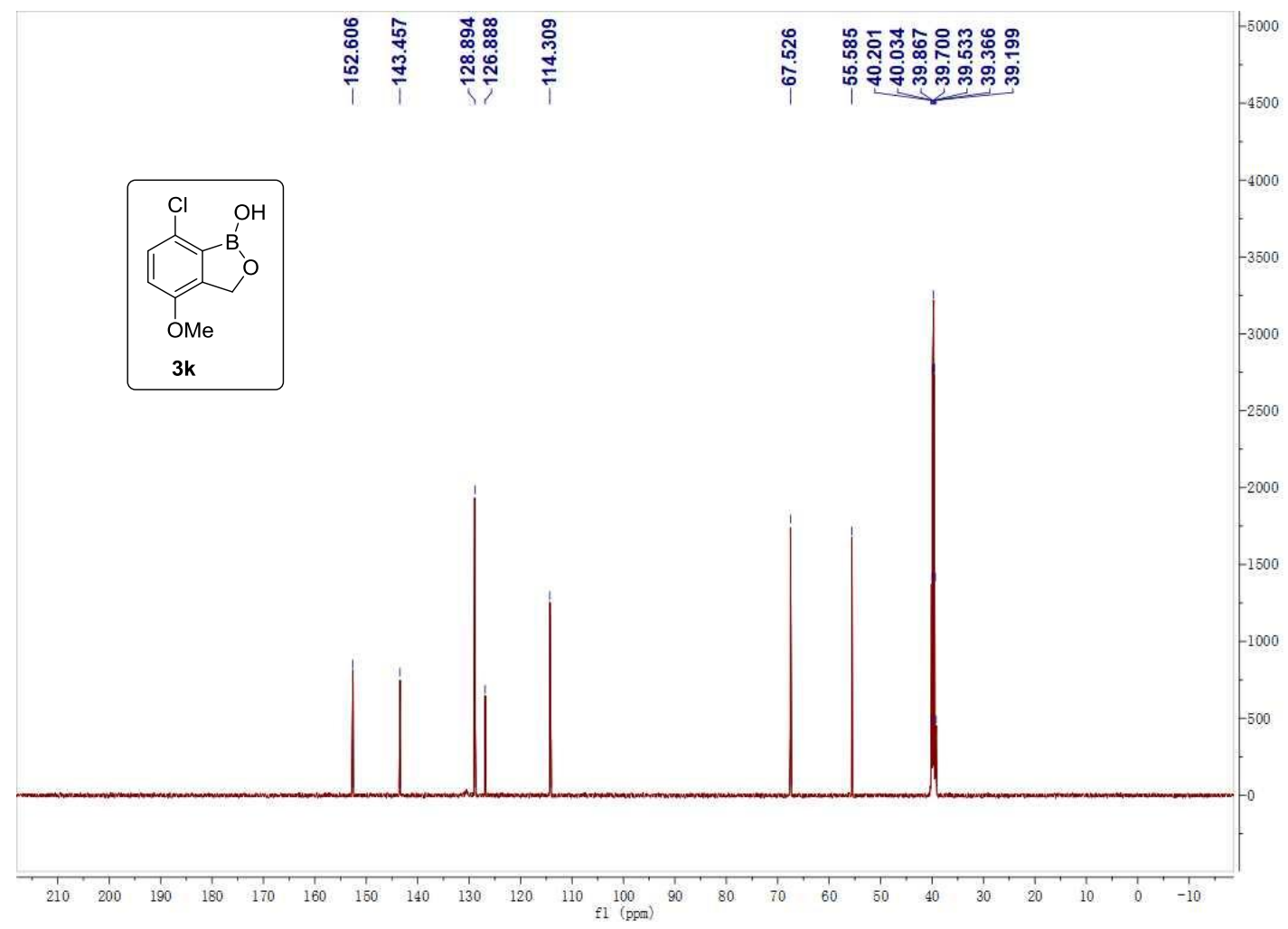


4,6-dimethylbenzo[c] [1,2] oxaborol-1(3H)-ol (3I)

${ }^{1}$ H NMR (400 MHz, DMSO-d6)

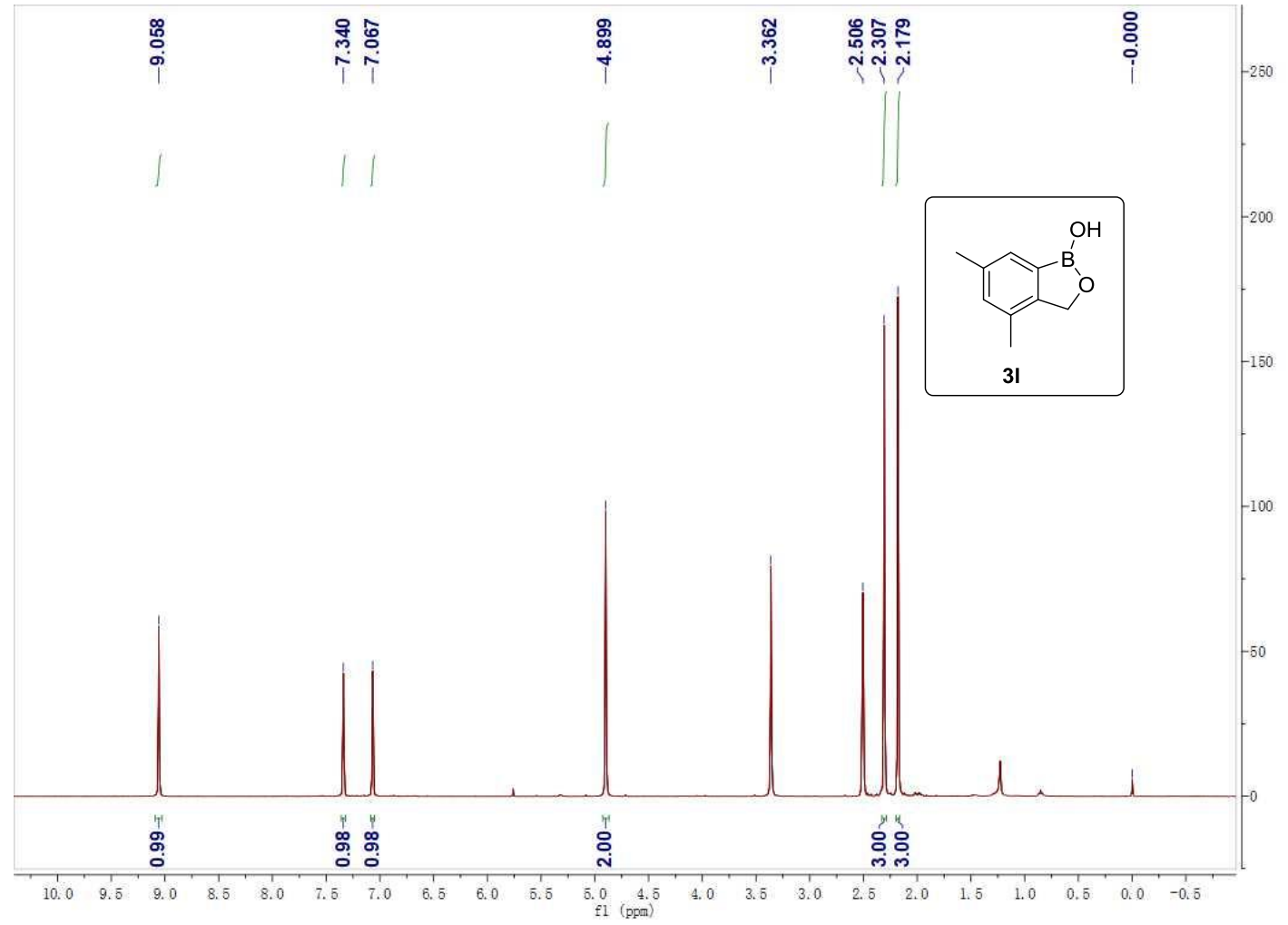

${ }^{13}$ C NMR (125 MHz, DMSO-d6)

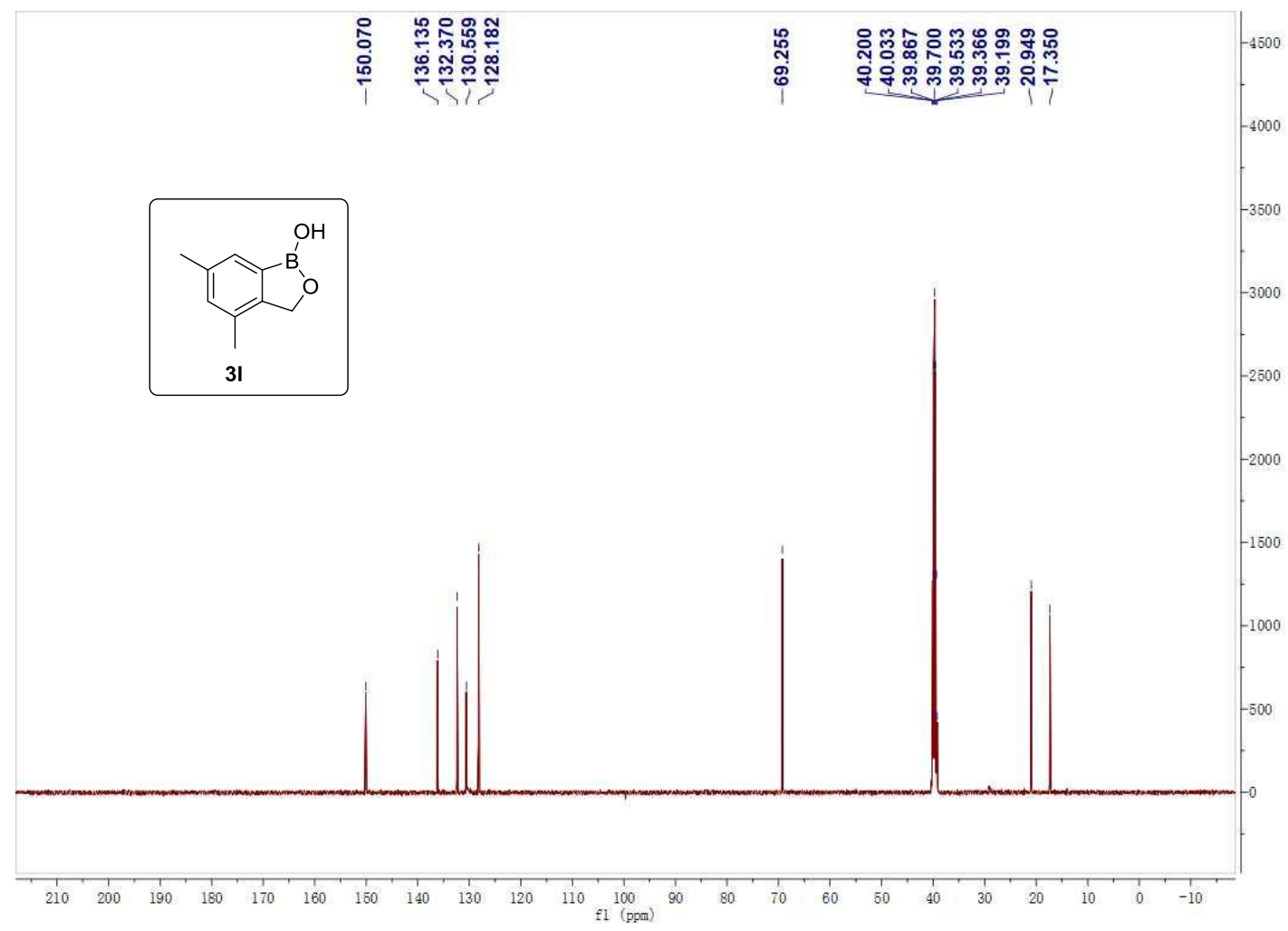


4,6-dimethoxy-3-methylbenzo[c] [1,2] oxaborol-1(3H)-ol (4a)

${ }^{1}$ H NMR (500 MHz, DMSO-d6)

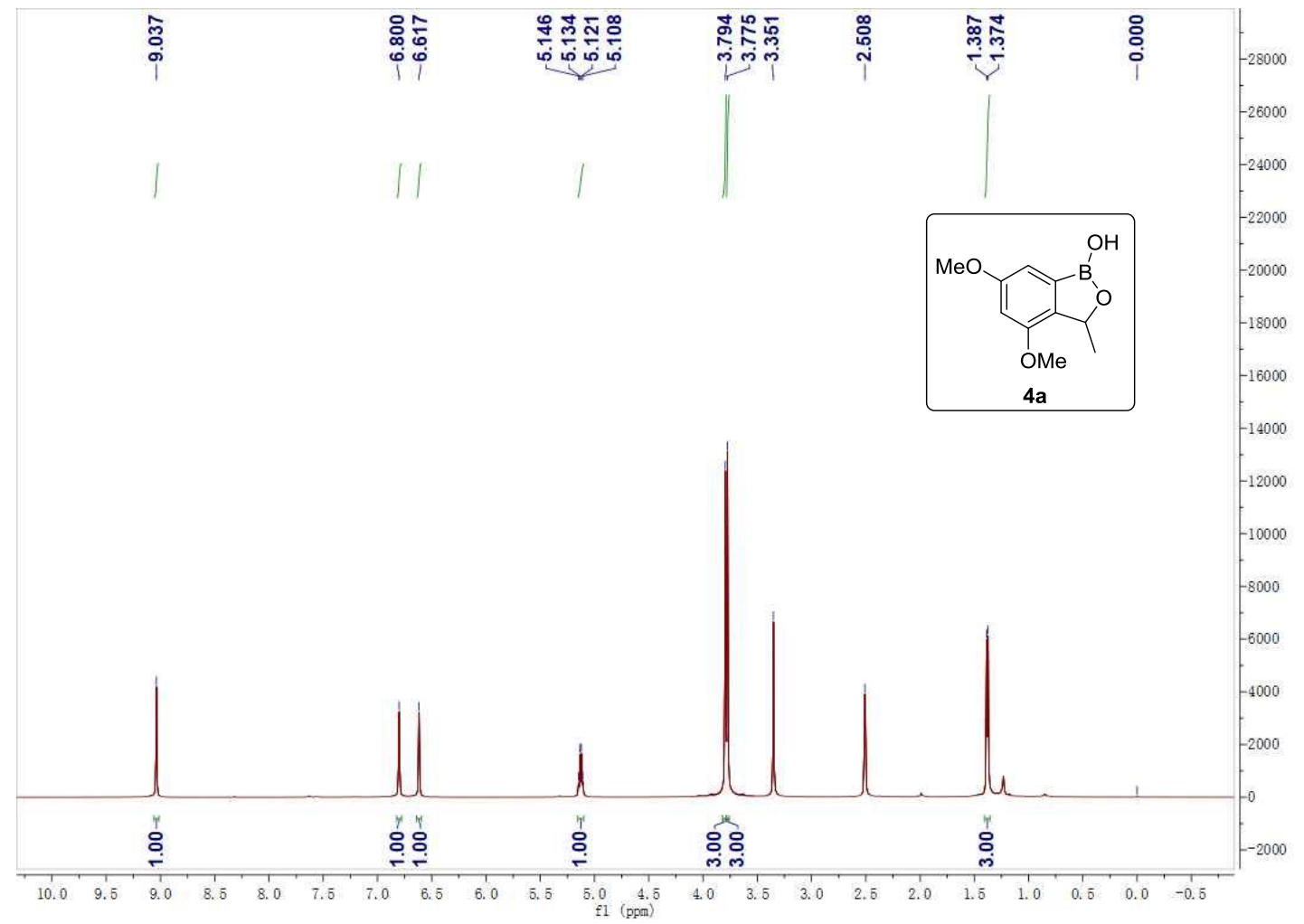

${ }^{13}$ C NMR (125 MHz, DMSO- $d 6$ )

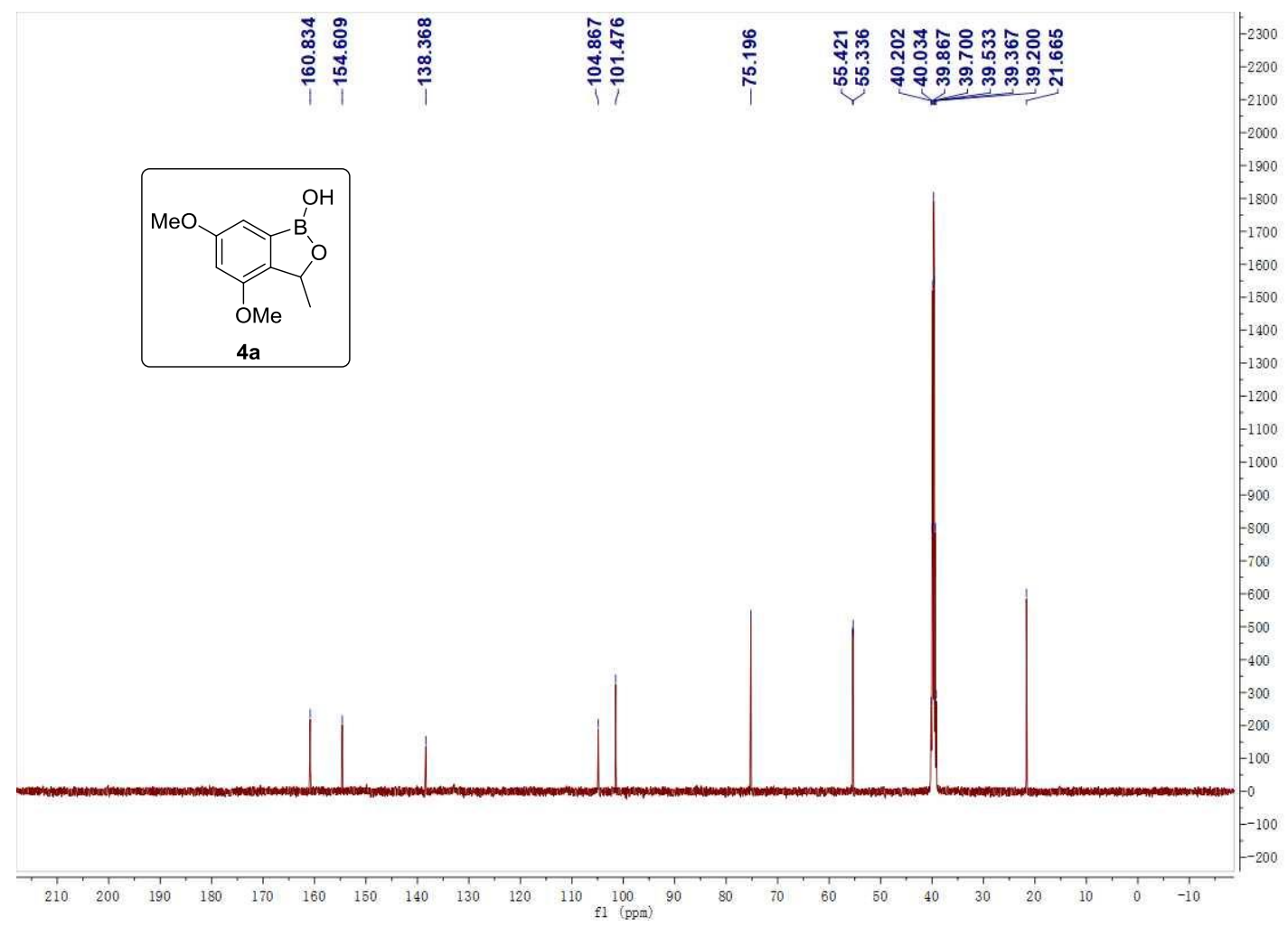


3-isobutyl-4,6-dimethoxybenzo[c] [1,2] oxaborol-1(3H)-ol (4b)

${ }^{\mathbf{1}} \mathbf{H}$ NMR (400 MHz, DMSO-d6)

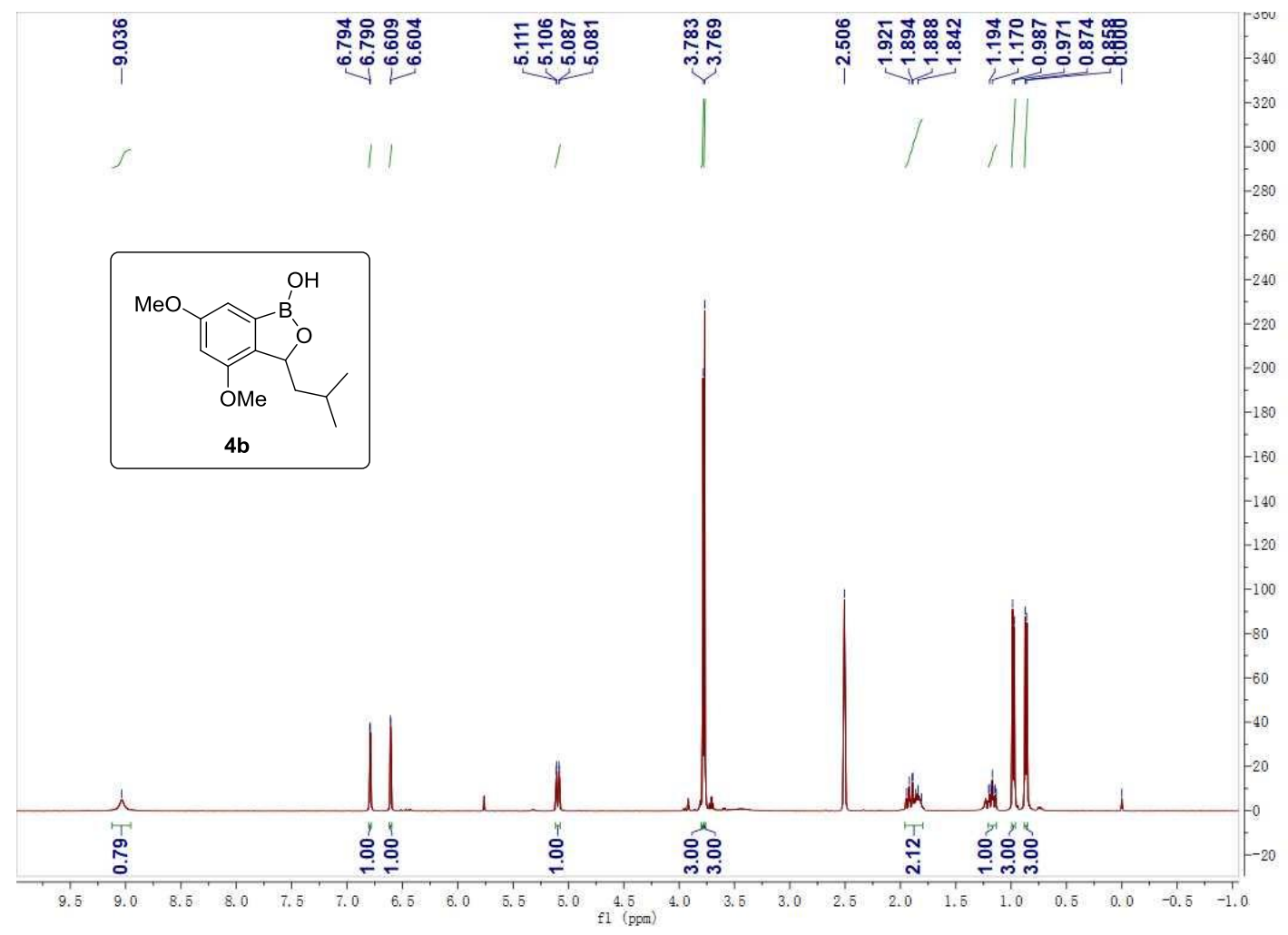

${ }^{13}$ C NMR (125 MHz, DMSO- $\left.d 6\right)$

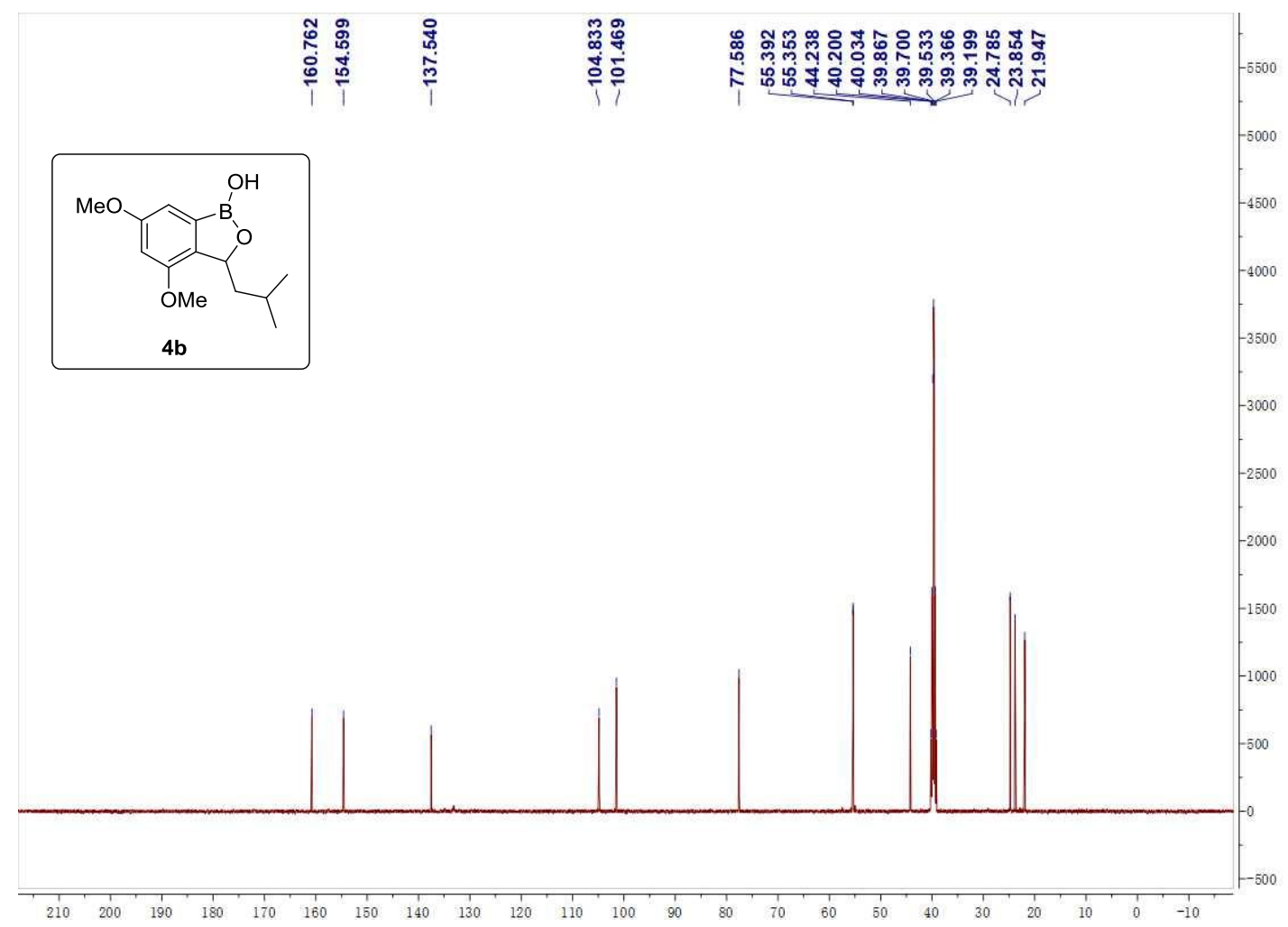


3-isopropyl-4,6-dimethoxybenzo[c] [1,2] oxaborol-1(3H)-ol (4c)

${ }^{\mathbf{1}} \mathbf{H}$ NMR $\left(400 \mathrm{MHz}, \mathrm{CDCl}_{3}\right)$

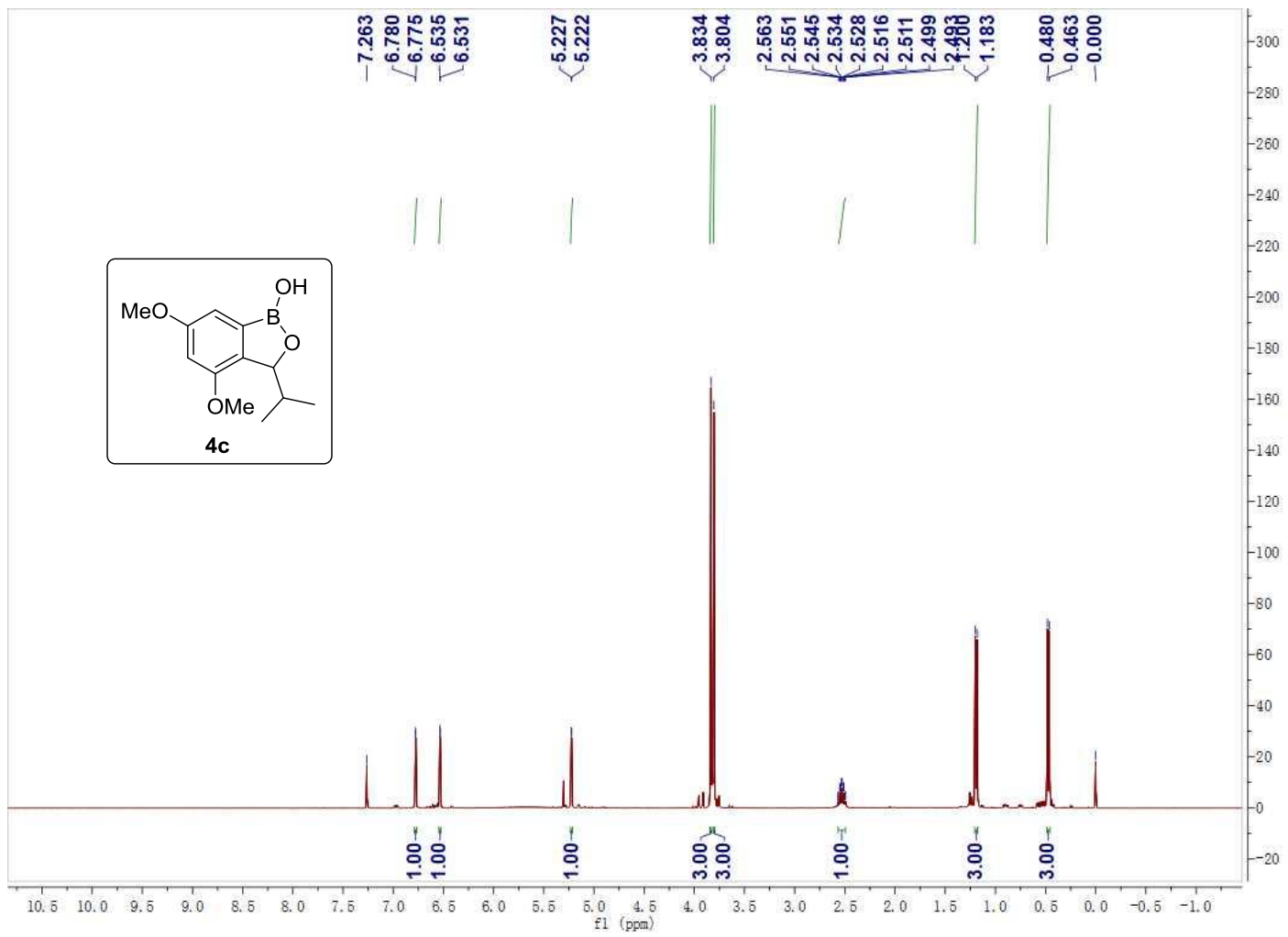

${ }^{1}$ H NMR (400 MHz, DMSO-d6)

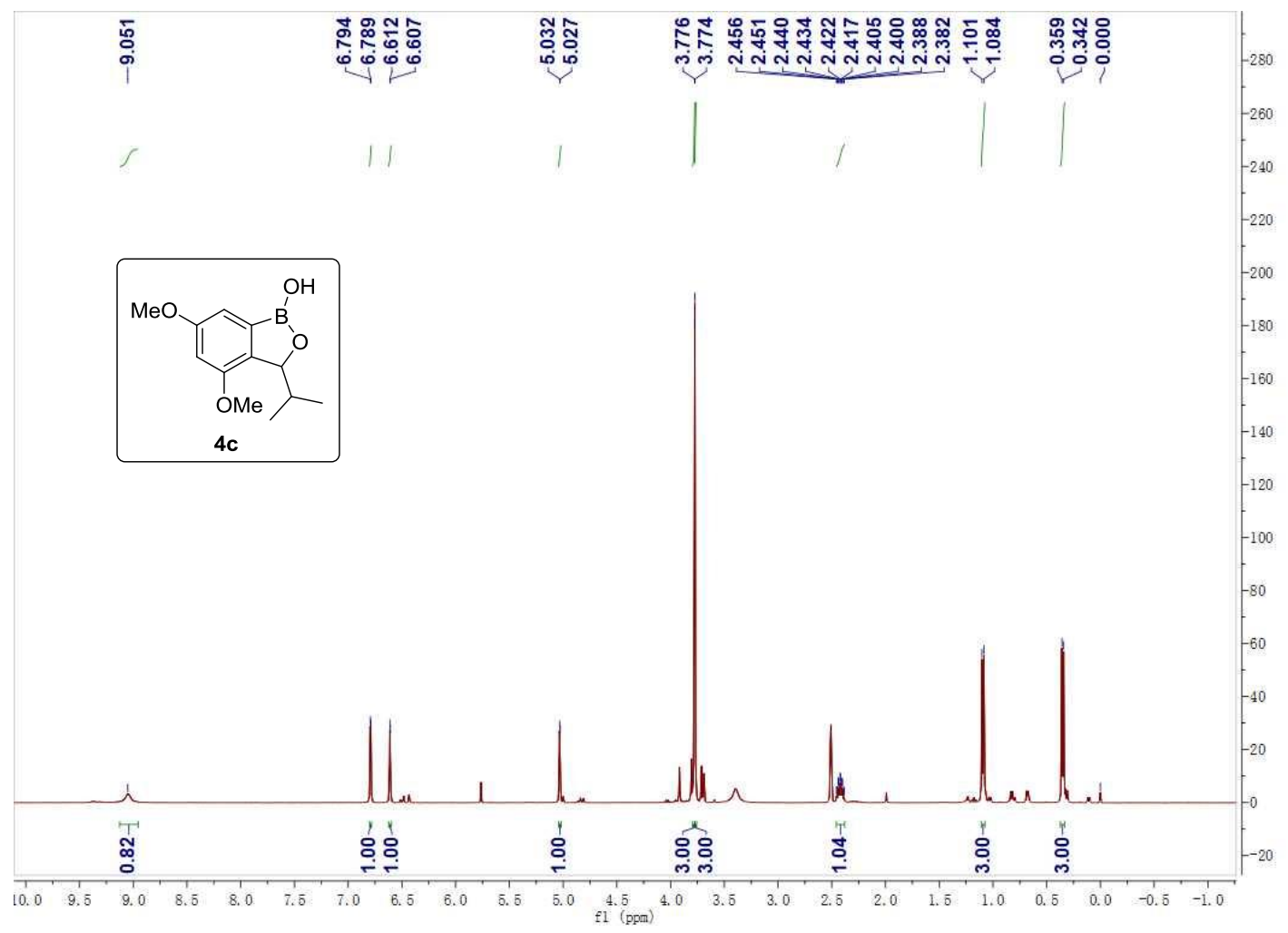


${ }^{13}$ C NMR (125 MHz, DMSO-d6)

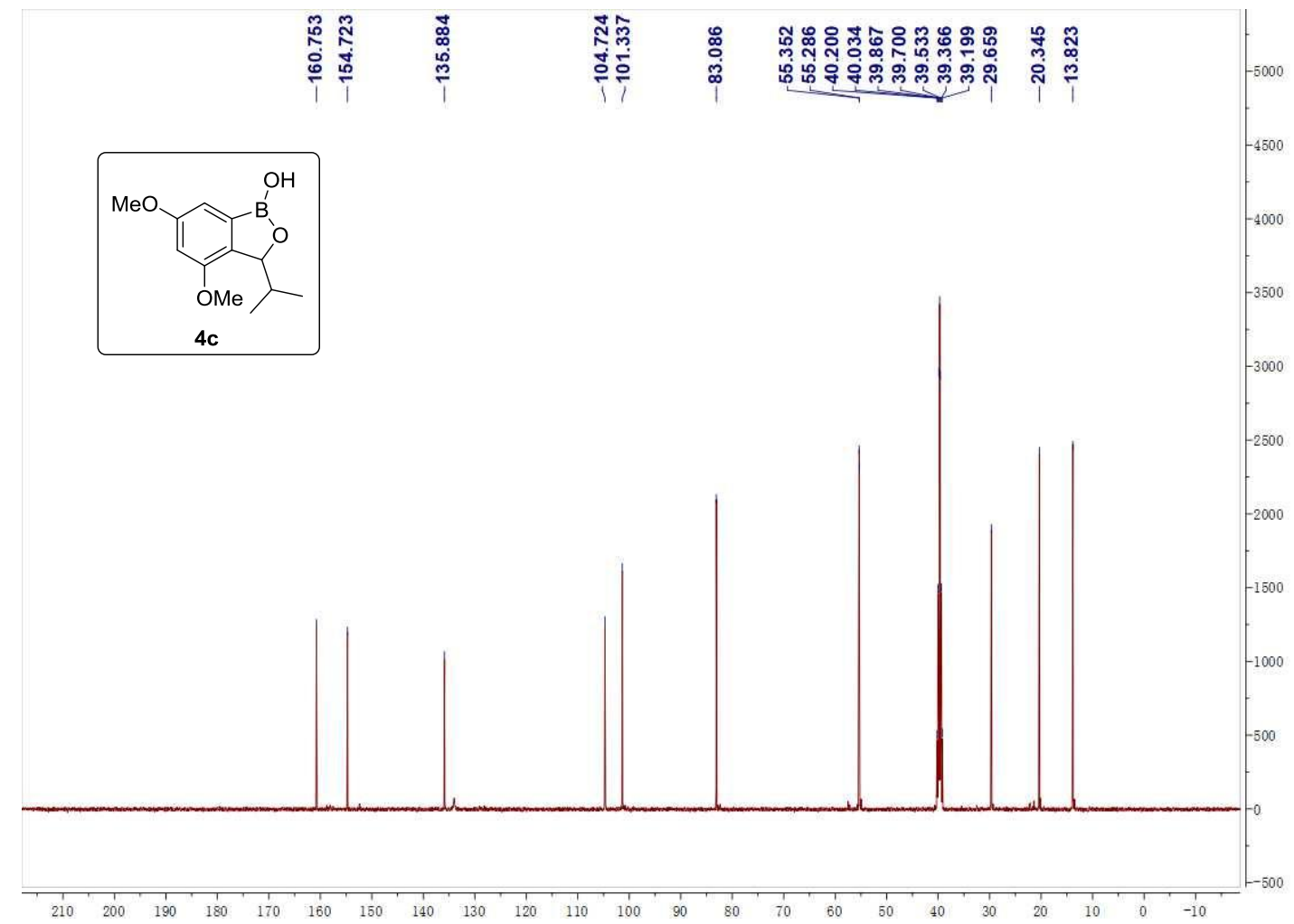


3-(benzyloxy)-4,6-dimethoxybenzo[c] [1,2] oxaborol-1(3H)-ol (4d)

${ }^{1}$ H NMR (400 MHz, DMSO-d6)

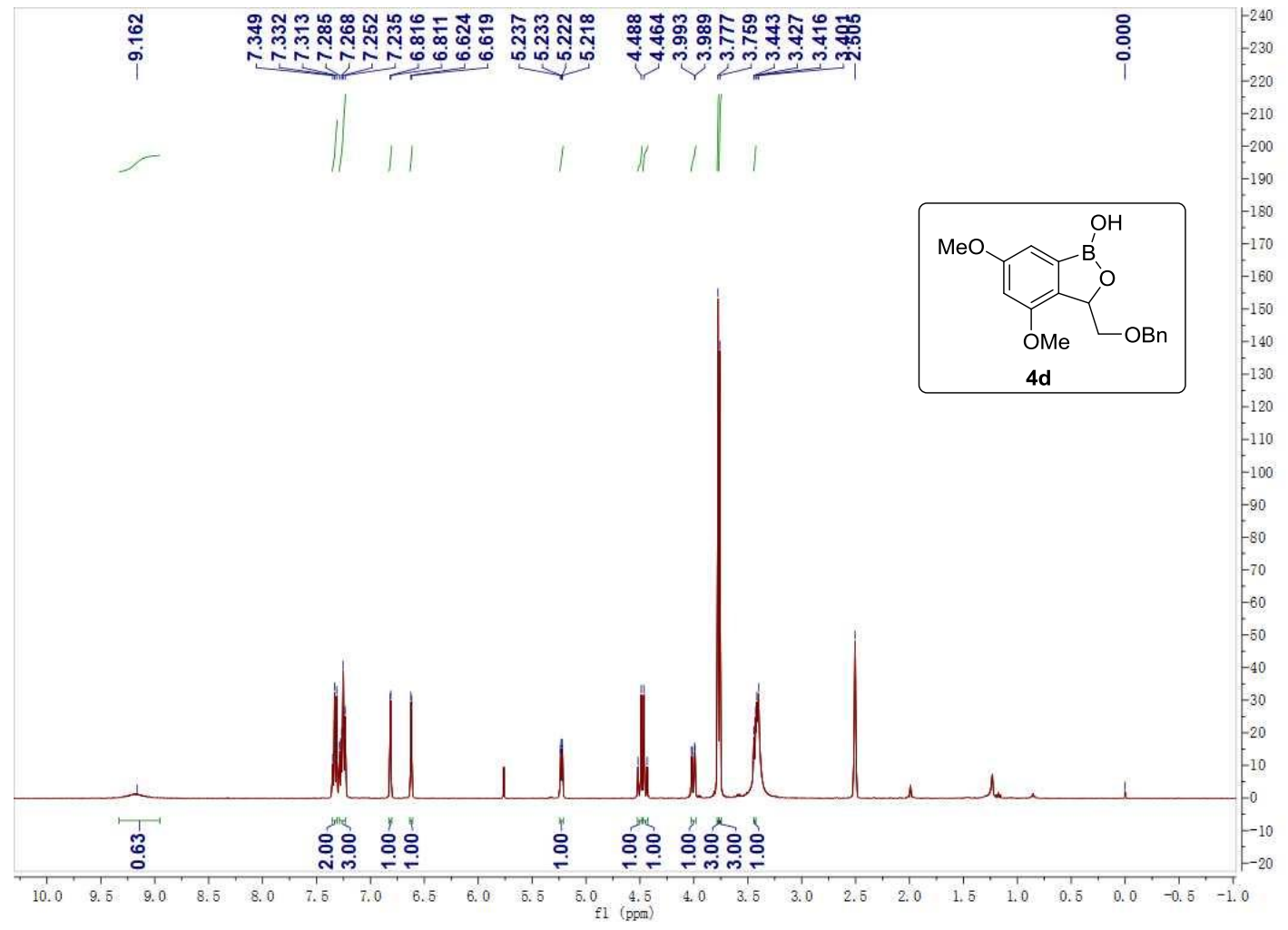

${ }^{13}$ C NMR (125 MHz, DMSO-d6)

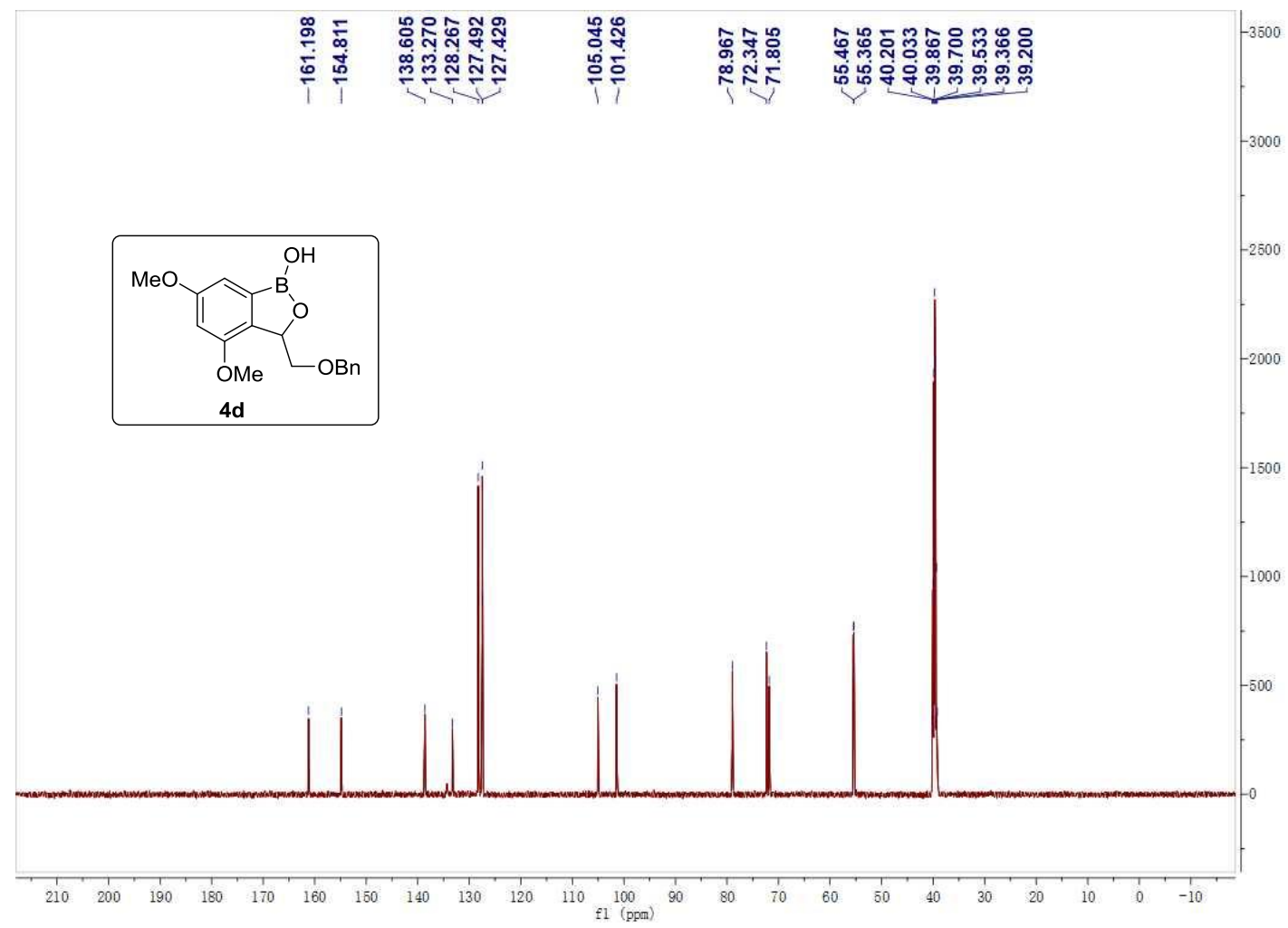


ethyl 1-hydroxy-4,6-dimethoxy-1,3-dihydrobenzo[c] [1,2] oxaborole-3-carboxylate (4e)

${ }^{1}$ H NMR (400 MHz, DMSO- $d 6$ )

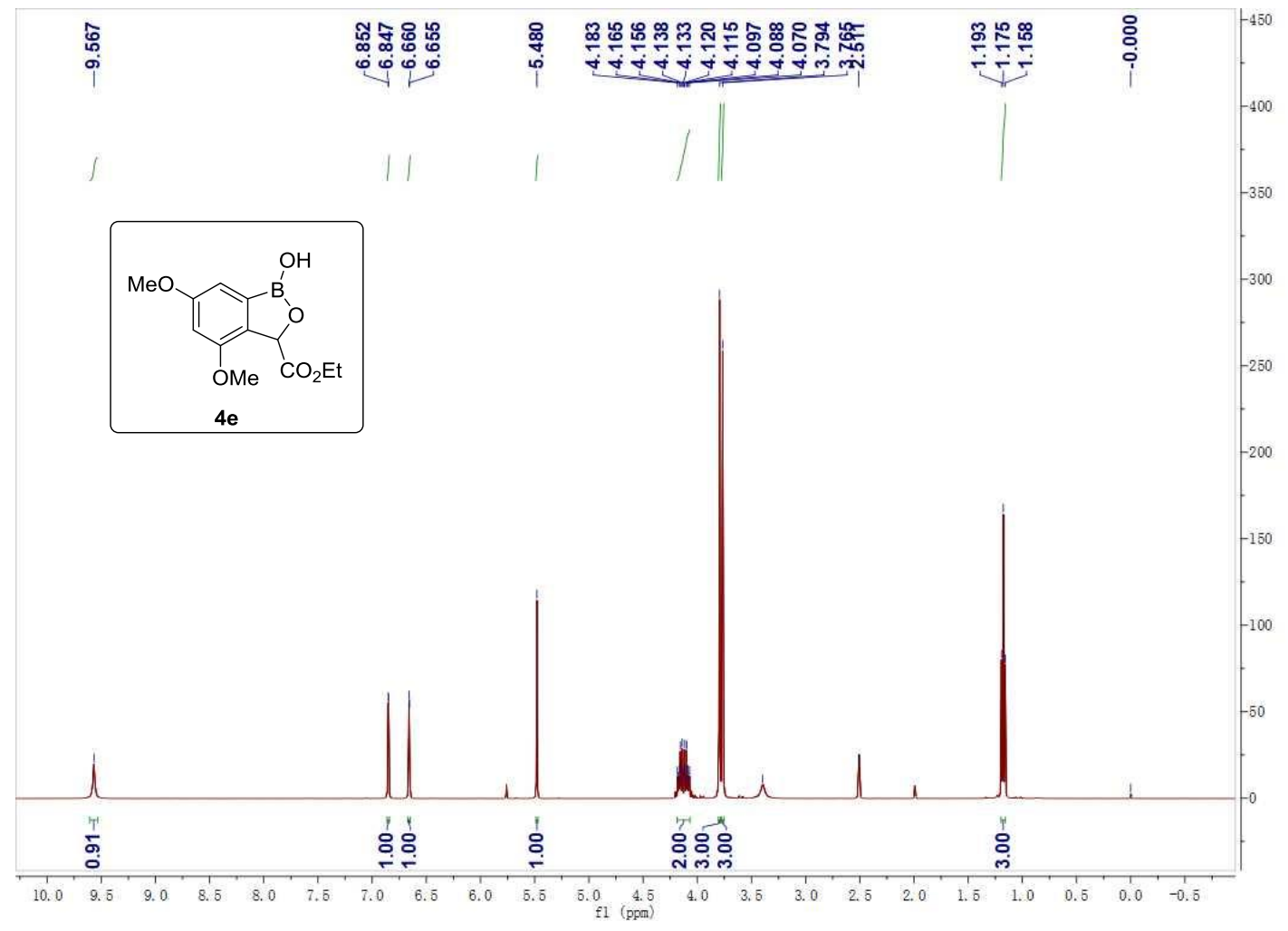

${ }^{13}$ C NMR (125 MHz, DMSO- $\left.d 6\right)$

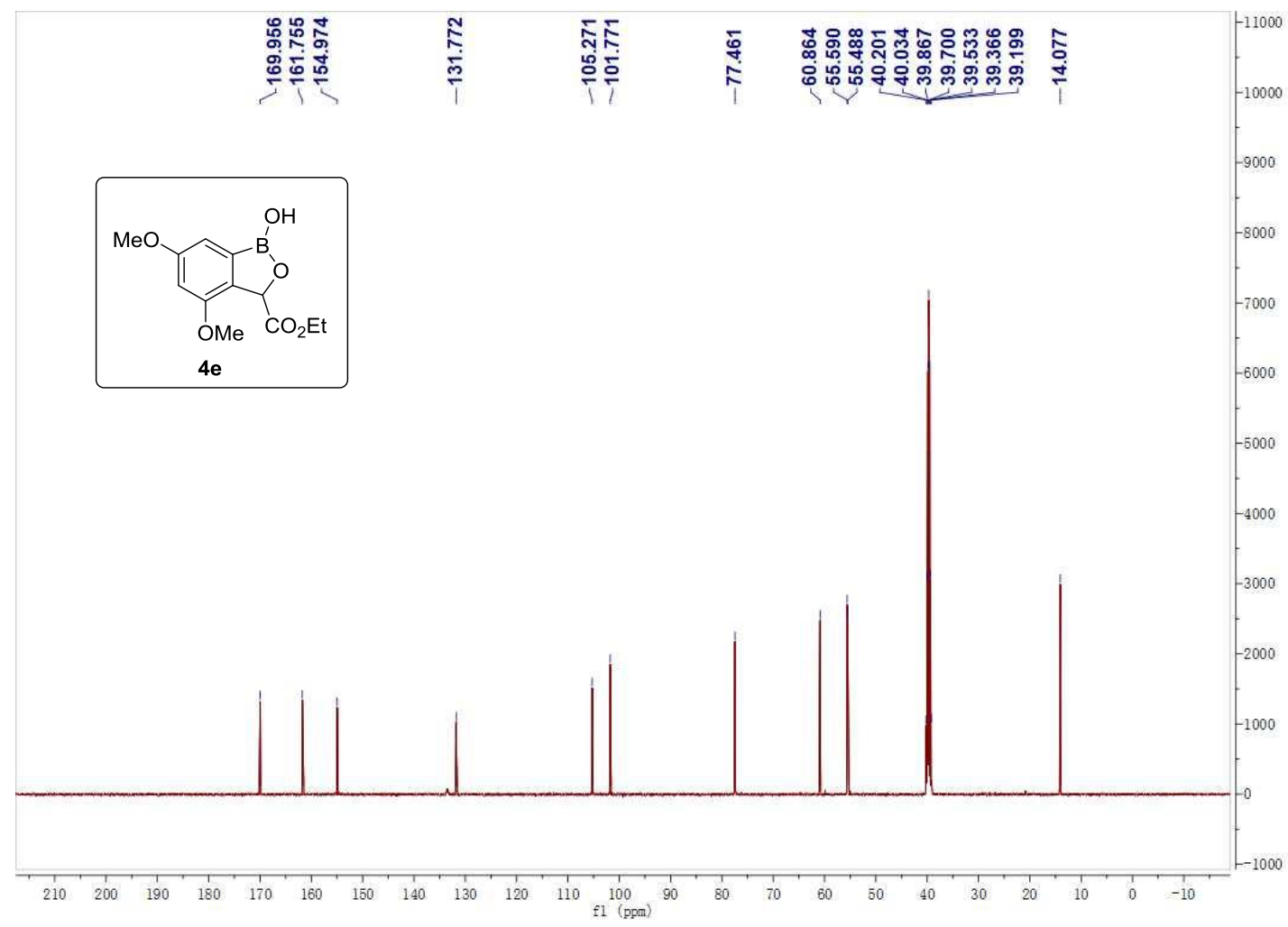


4,6-dimethoxy-3-(trifluoromethyl) benzo[c] [1,2] oxaborol-1(3H)-ol (4f)

${ }^{1}$ H NMR (400 MHz, DMSO-d6)

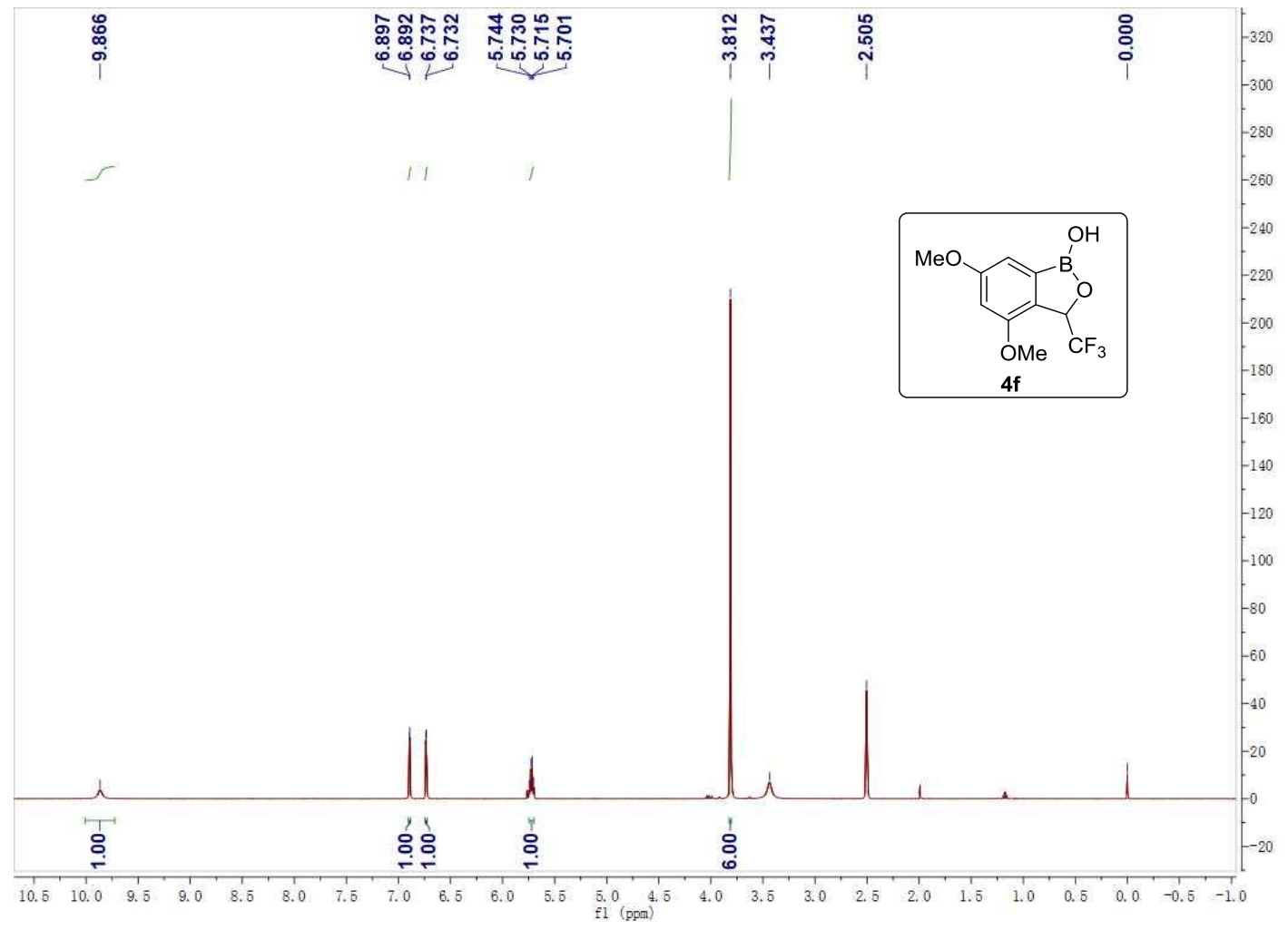

${ }^{13}$ C NMR (125 MHz, DMSO- $\left.d 6\right)$

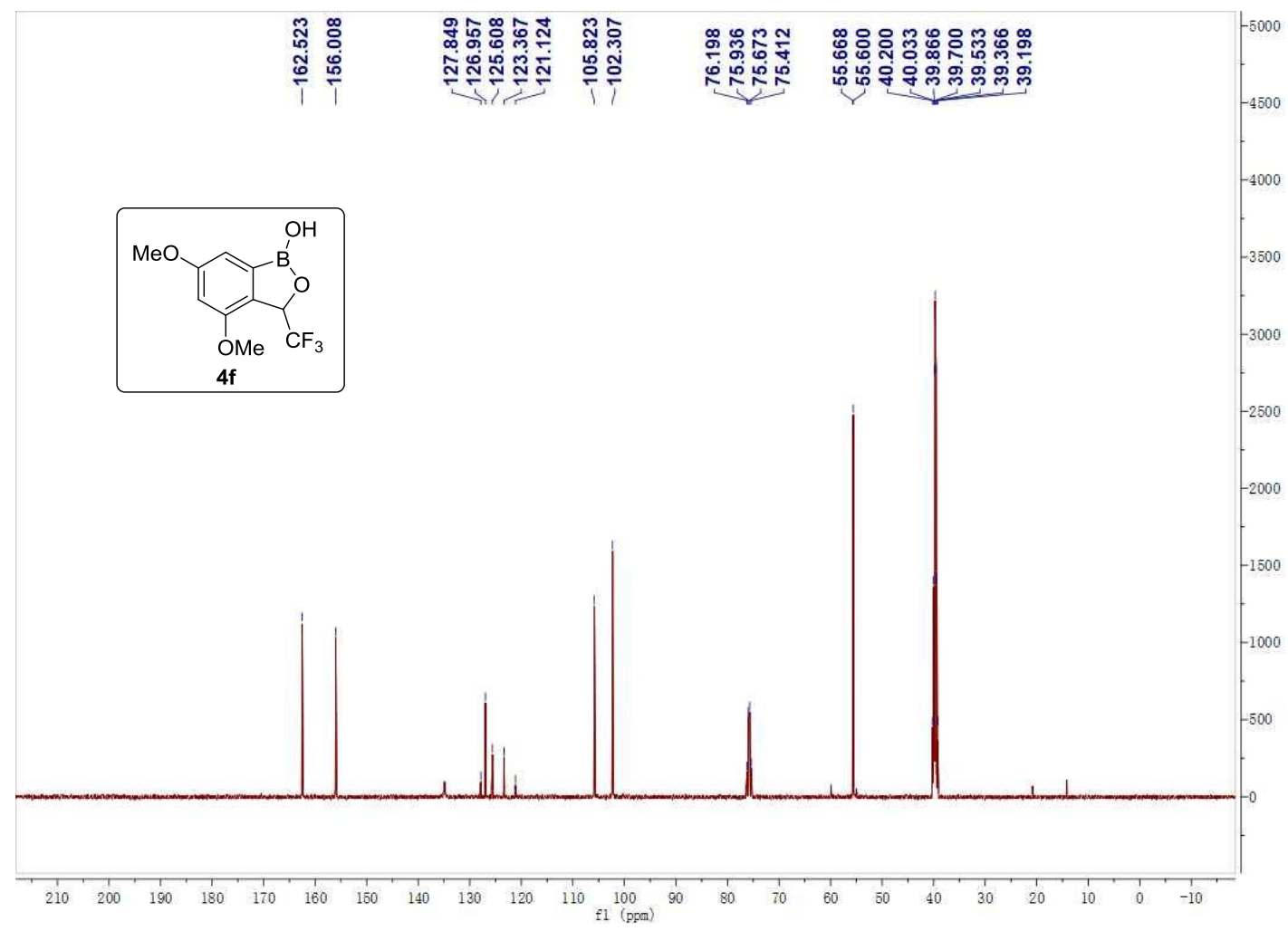


4,6-dimethoxy-3-phenylbenzo[c] [1,2] oxaborol-1(3H)-ol (4g)

${ }^{\mathbf{1}} \mathbf{H}$ NMR (400 MHz, DMSO-d6)

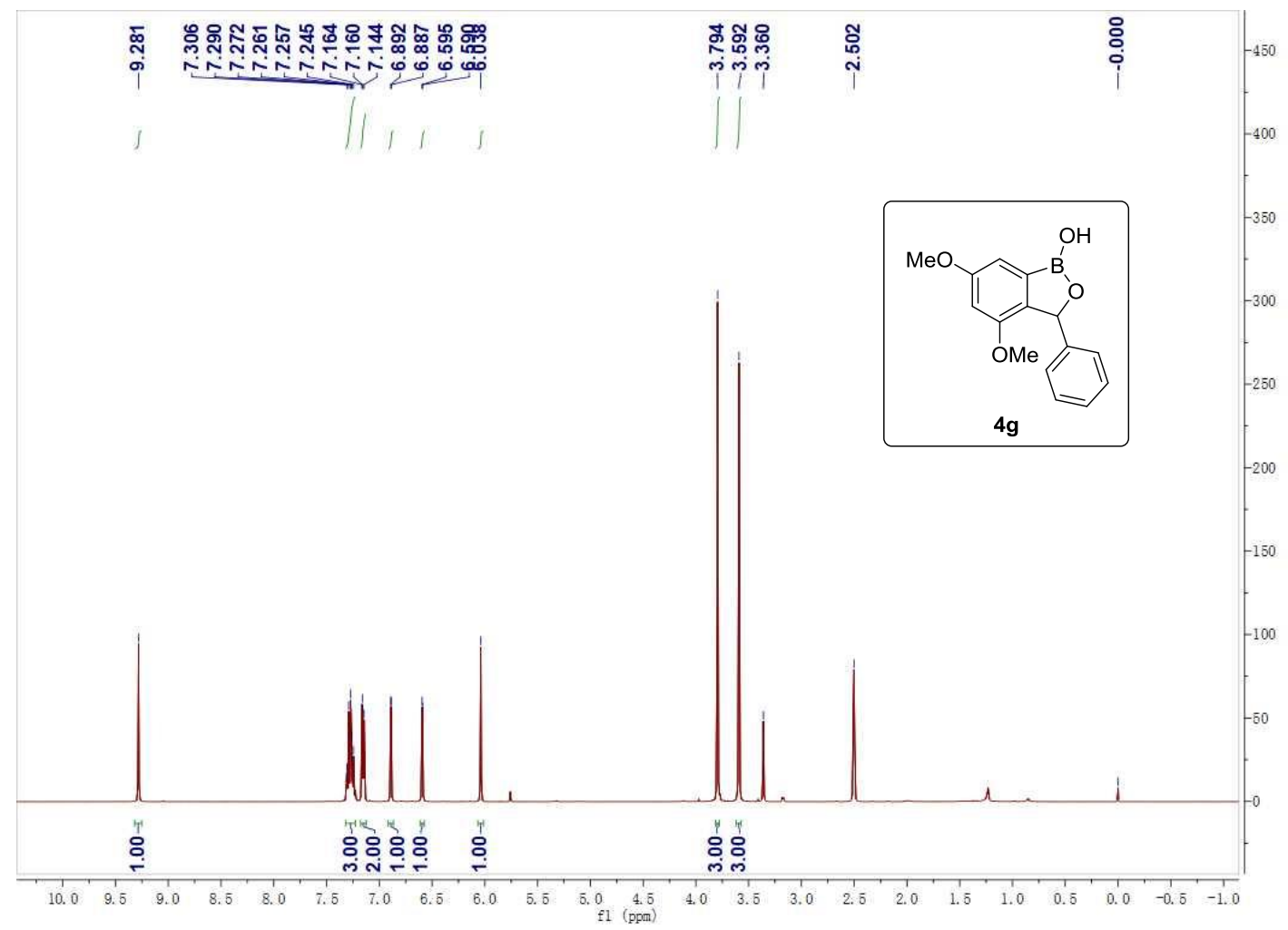

${ }^{13}$ C NMR (125 MHz, DMSO- $\left.d 6\right)$

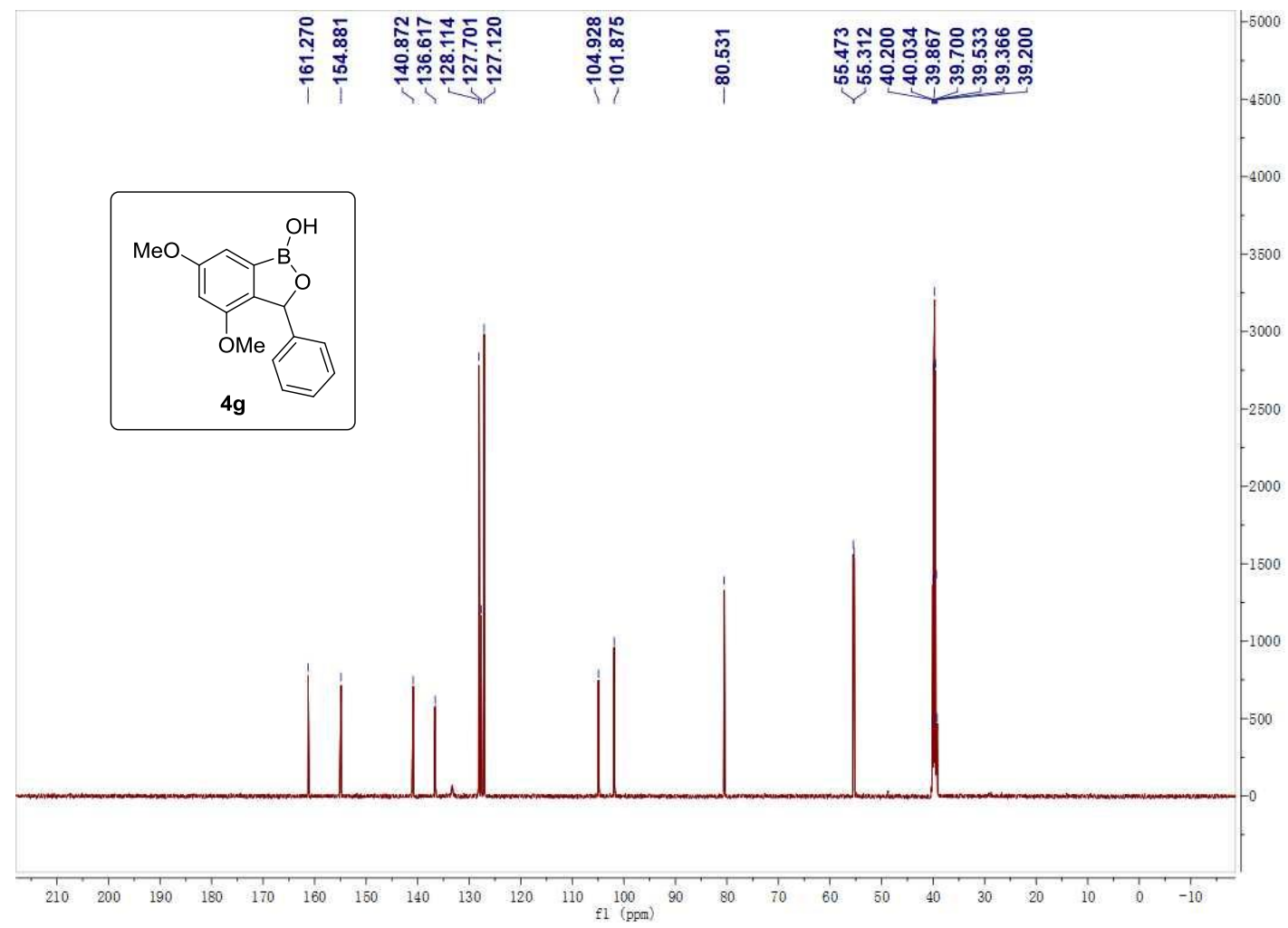


4,6-dimethoxy-3-(p-tolyl) benzo[c] [1,2] oxaborol-1(3H)-ol (4h)

${ }^{1}$ H NMR (400 MHz, DMSO-d6)

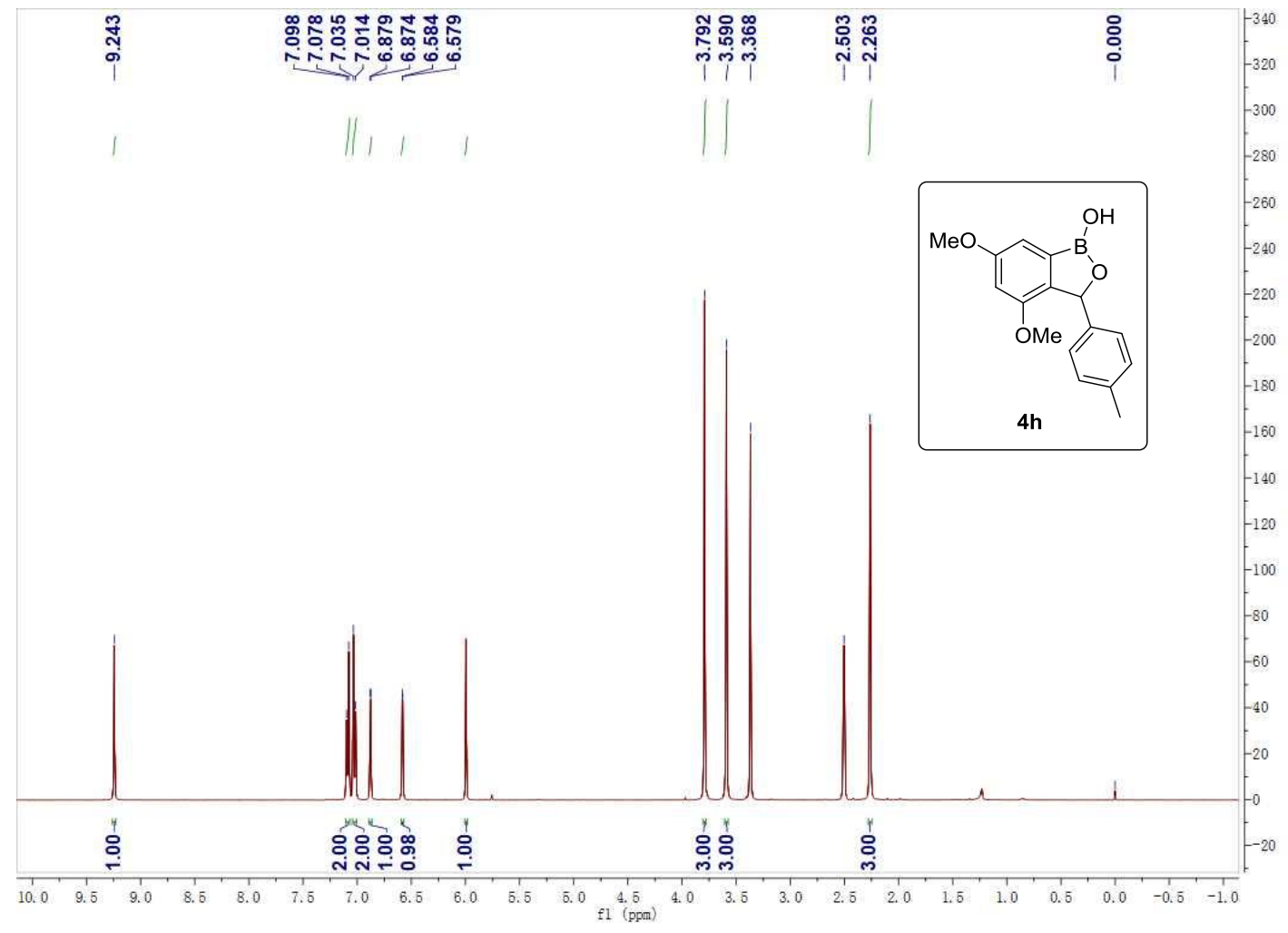

${ }^{13}$ C NMR (125 MHz, DMSO-d6)

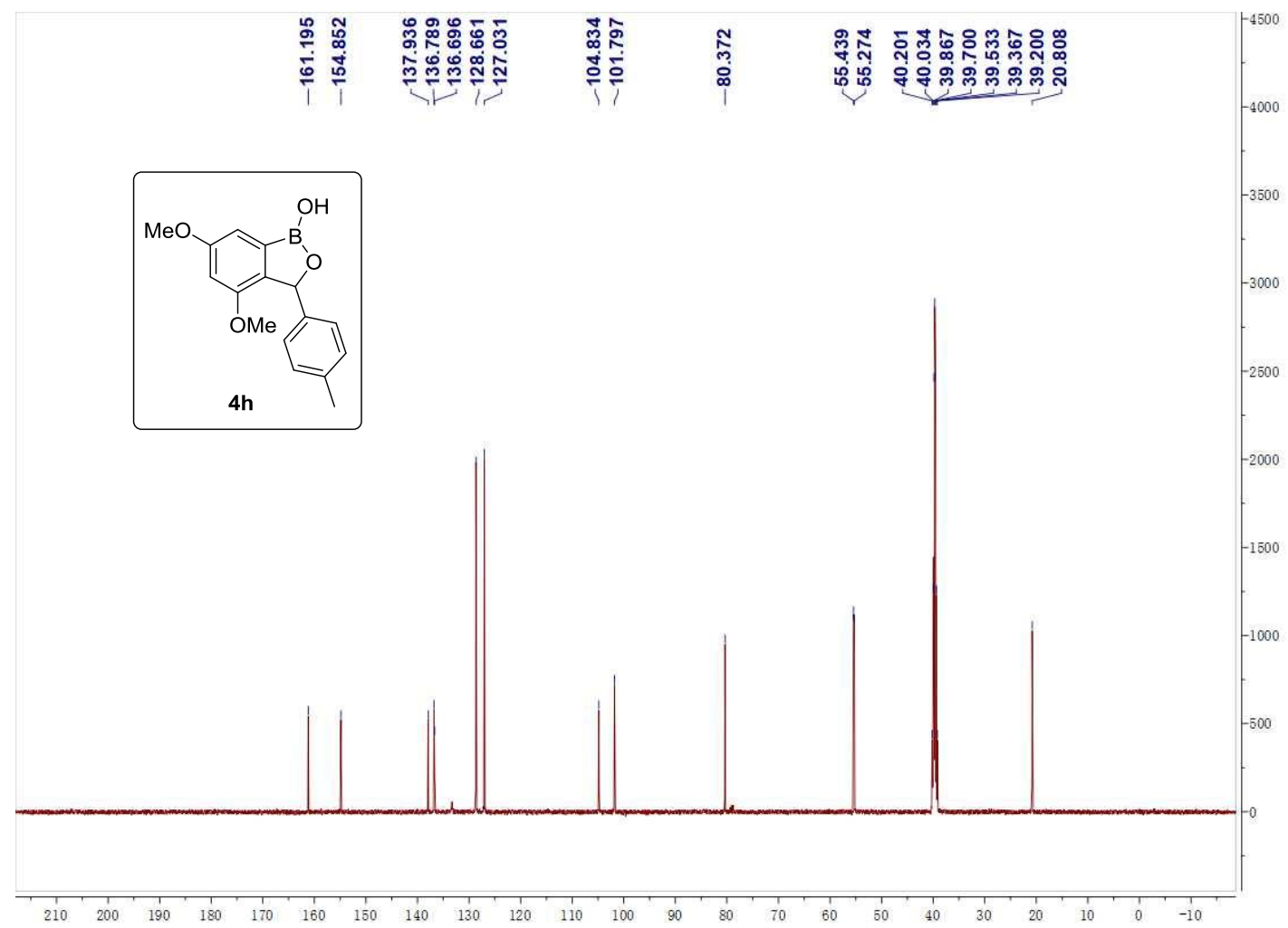


3-(4-fluorophenyl)-4,6-dimethoxybenzo[c] [1,2] oxaborol-1(3H)-ol (4i)

${ }^{1}$ H NMR (400 MHz, DMSO-d6)

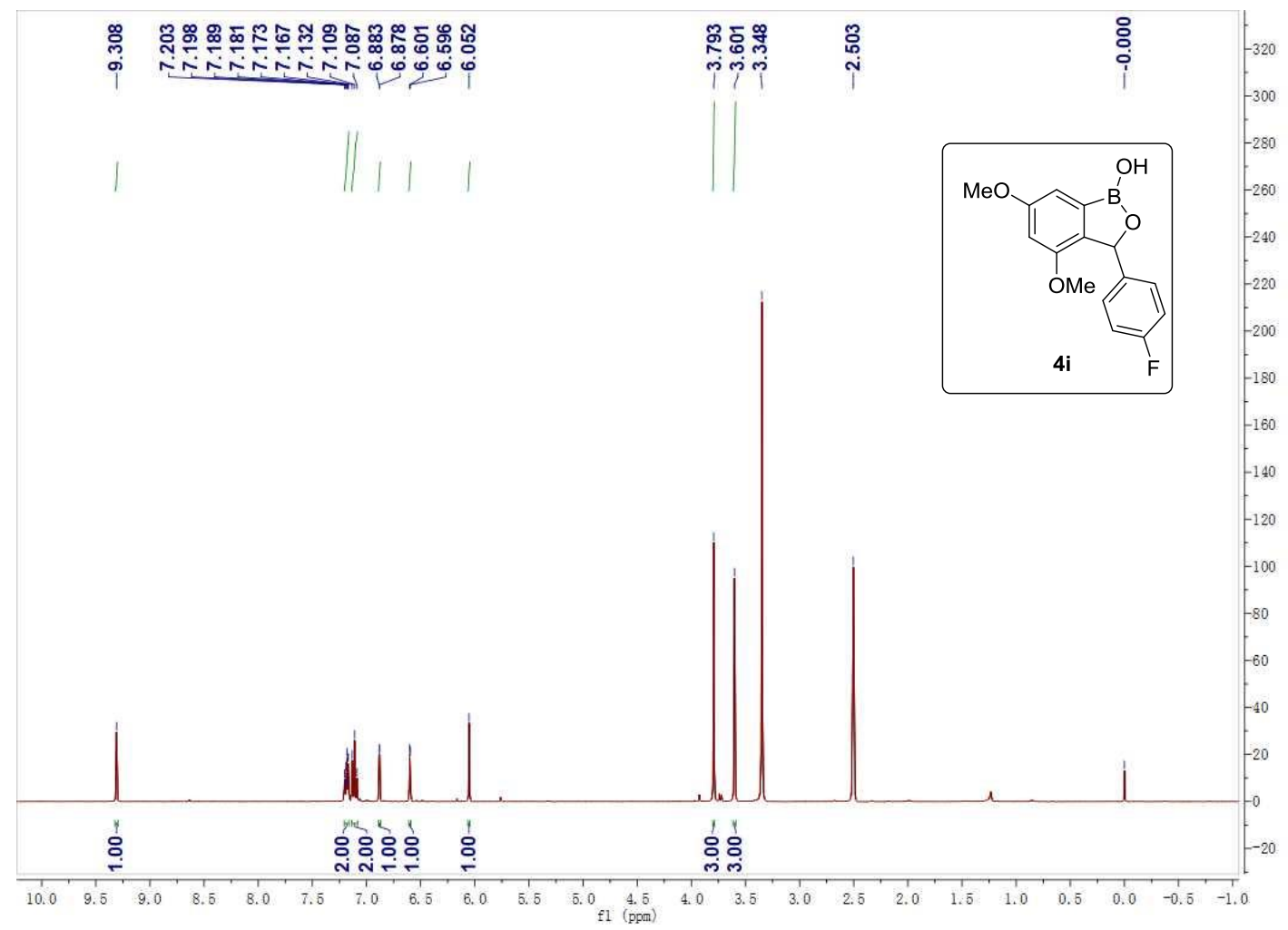

${ }^{13}$ C NMR (125 MHz, DMSO- $\left.d 6\right)$

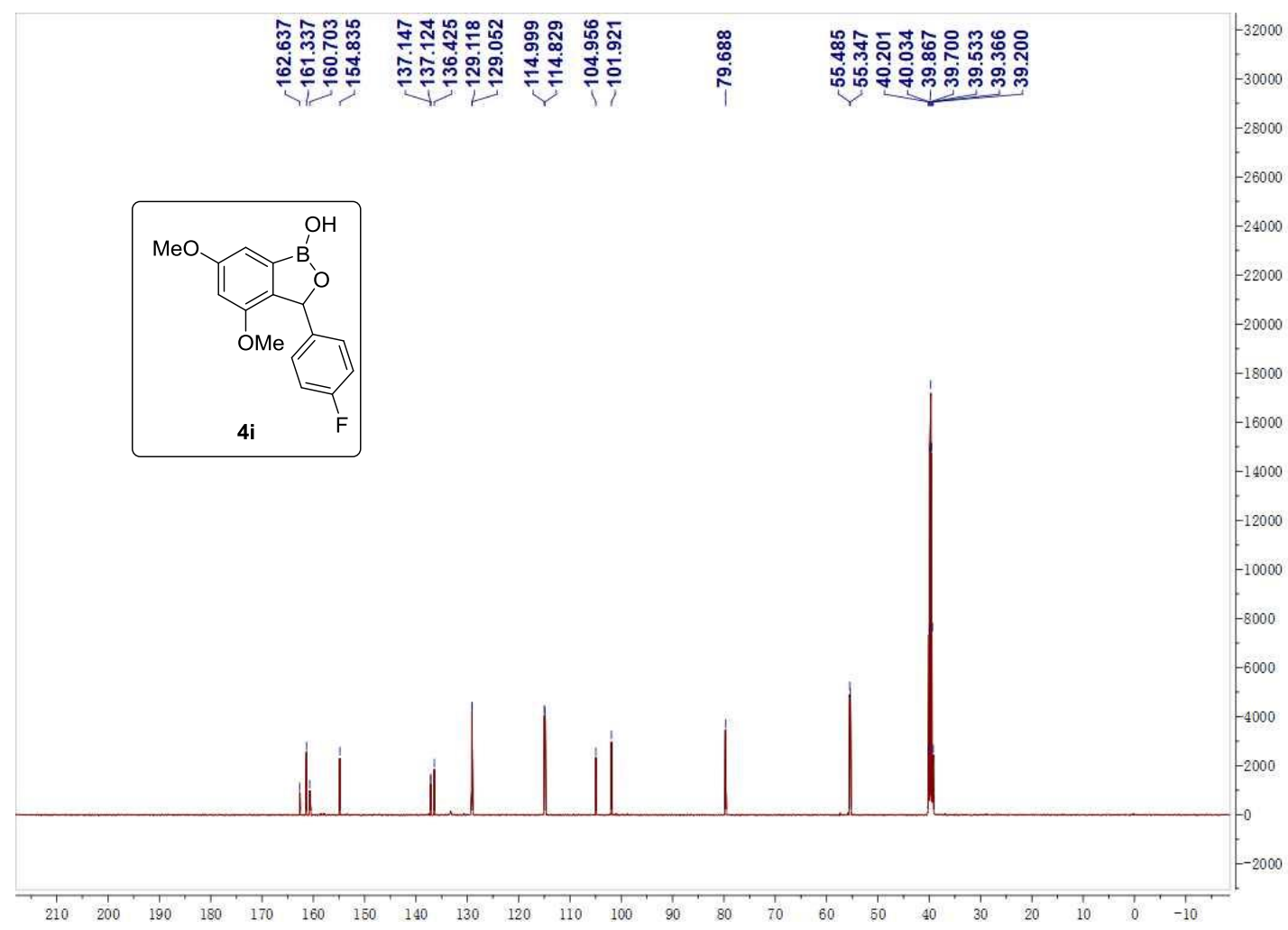


3-(4-chlorophenyl)-4,6-dimethoxybenzo[c] [1,2] oxaborol-1(3H)-ol (4j)

${ }^{1}$ H NMR (400 MHz, DMSO-d6)

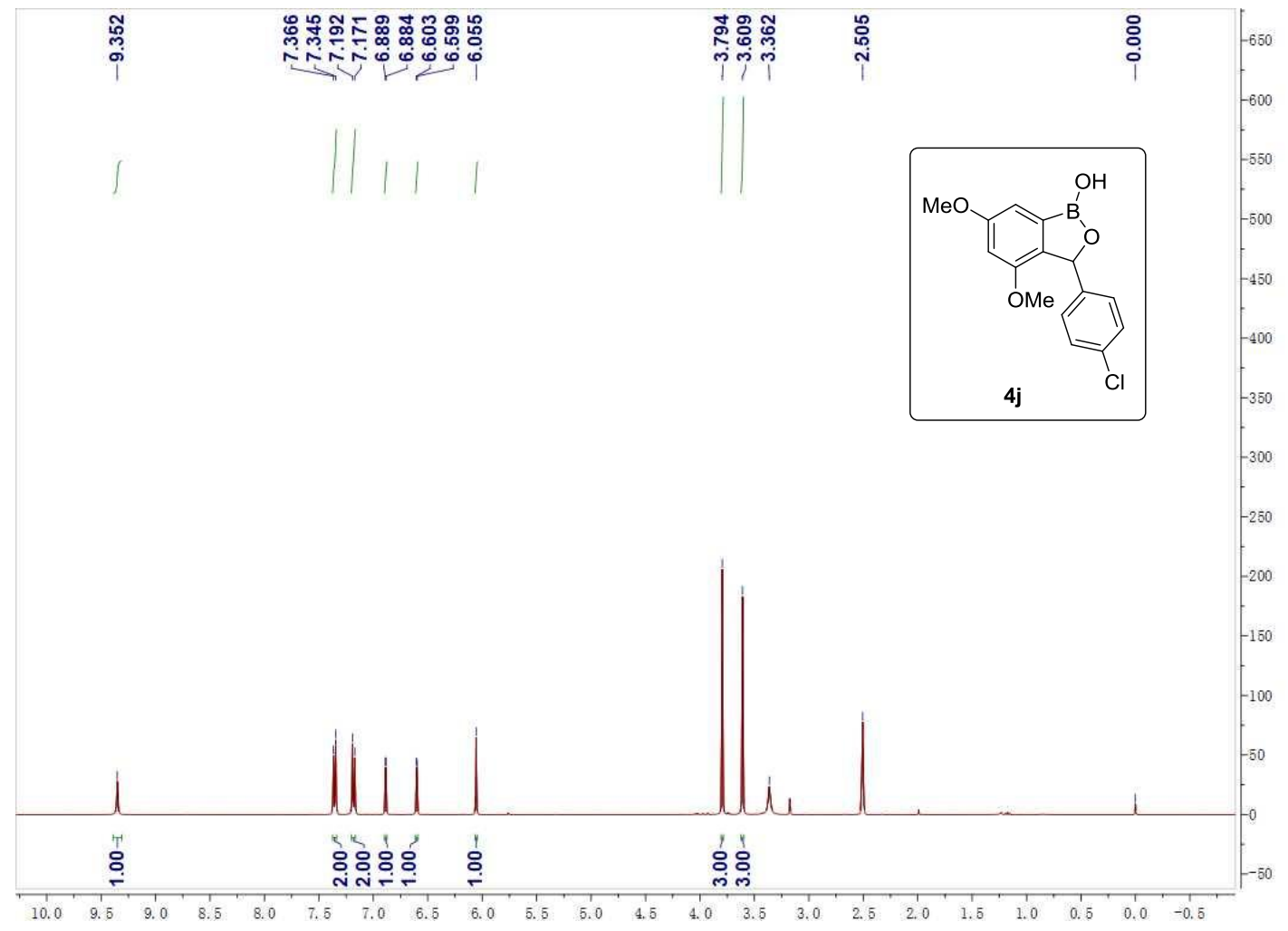

${ }^{13}$ C NMR (125 MHz, DMSO- $\left.d 6\right)$

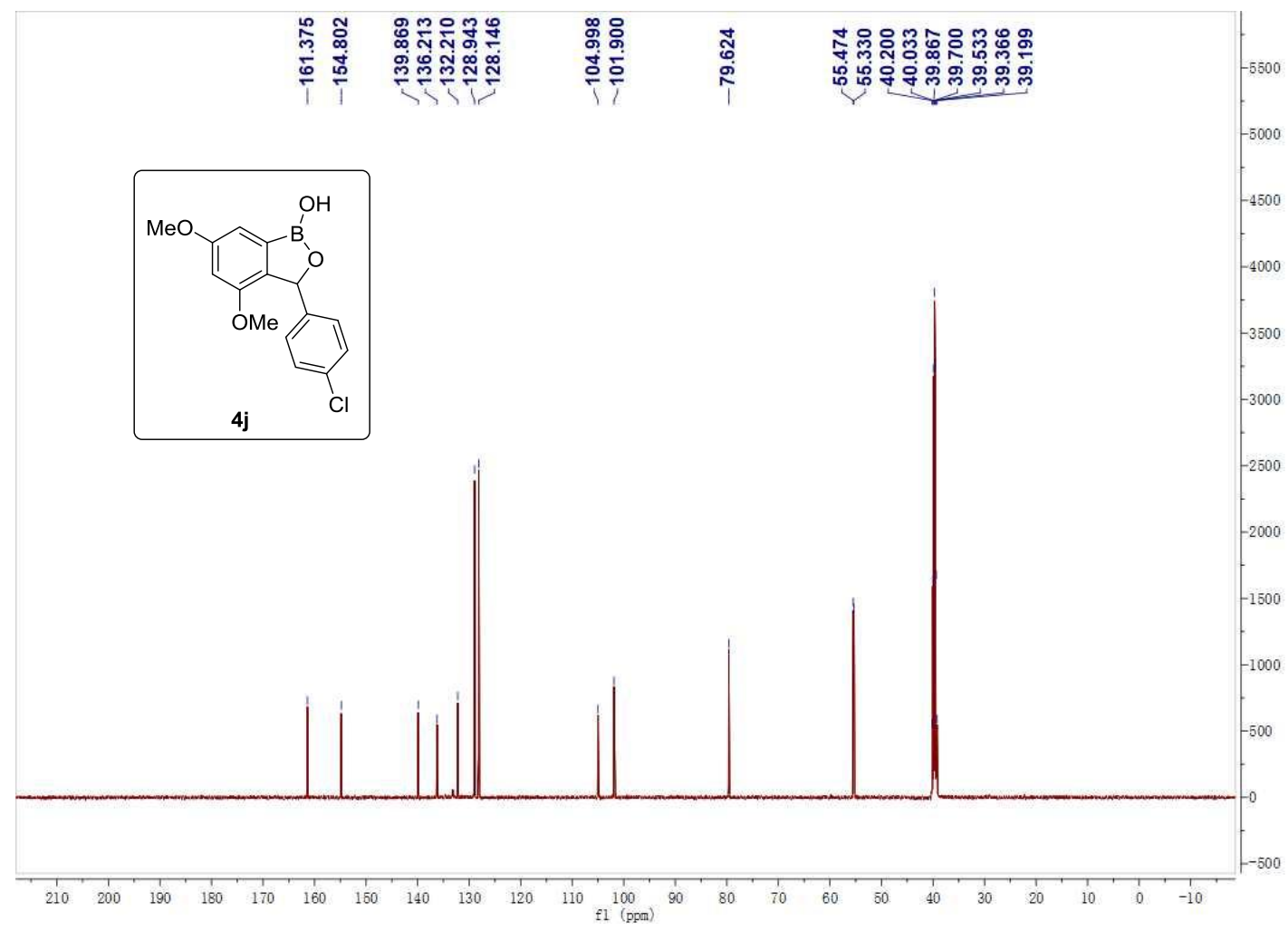


3-(4-bromophenyl)-4,6-dimethoxybenzo[c] [1,2] oxaborol-1(3H)-ol (4k)

${ }^{1}$ H NMR (500 MHz, DMSO-d6)

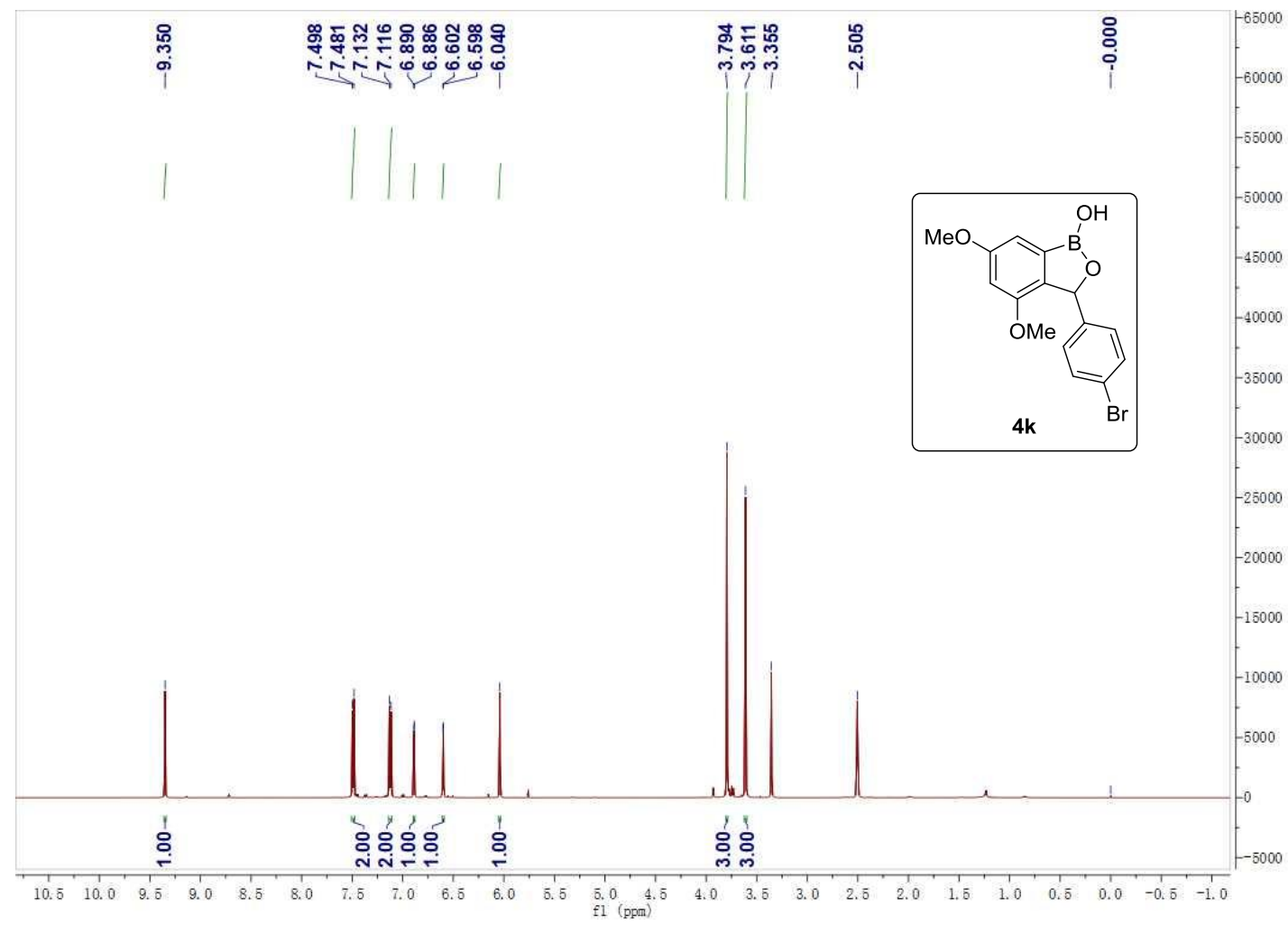

${ }^{13}$ C NMR (125 MHz, DMSO-d6)

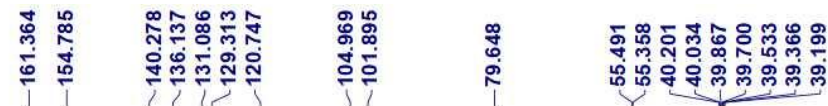

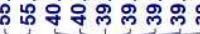

$-4000$

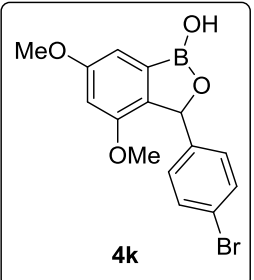

$4 k$

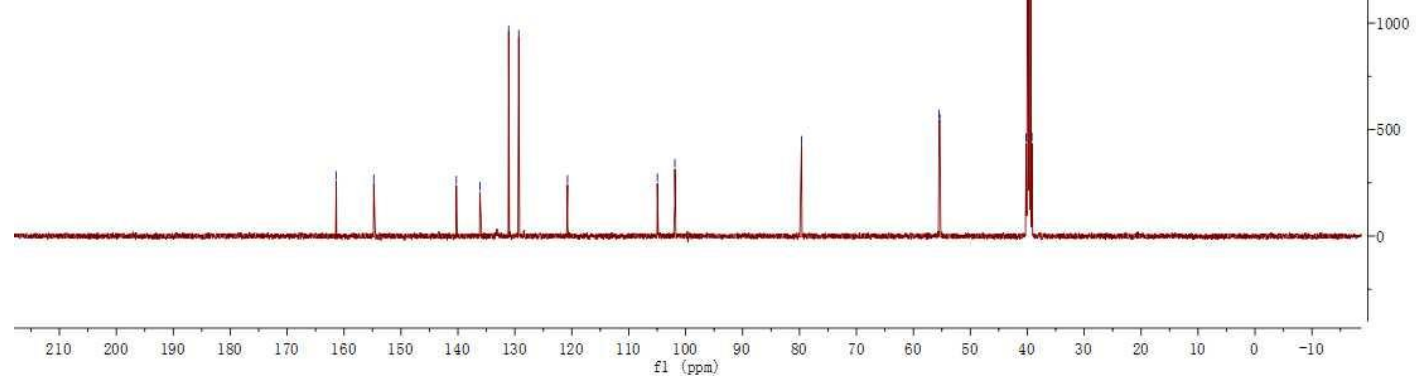


4,6-dimethoxy-3-(4-(trifluoromethyl) phenyl) benzo[c] [1,2] oxaborol-1(3H)-ol (4I)

${ }^{1}$ H NMR (400 MHz, DMSO-d6)

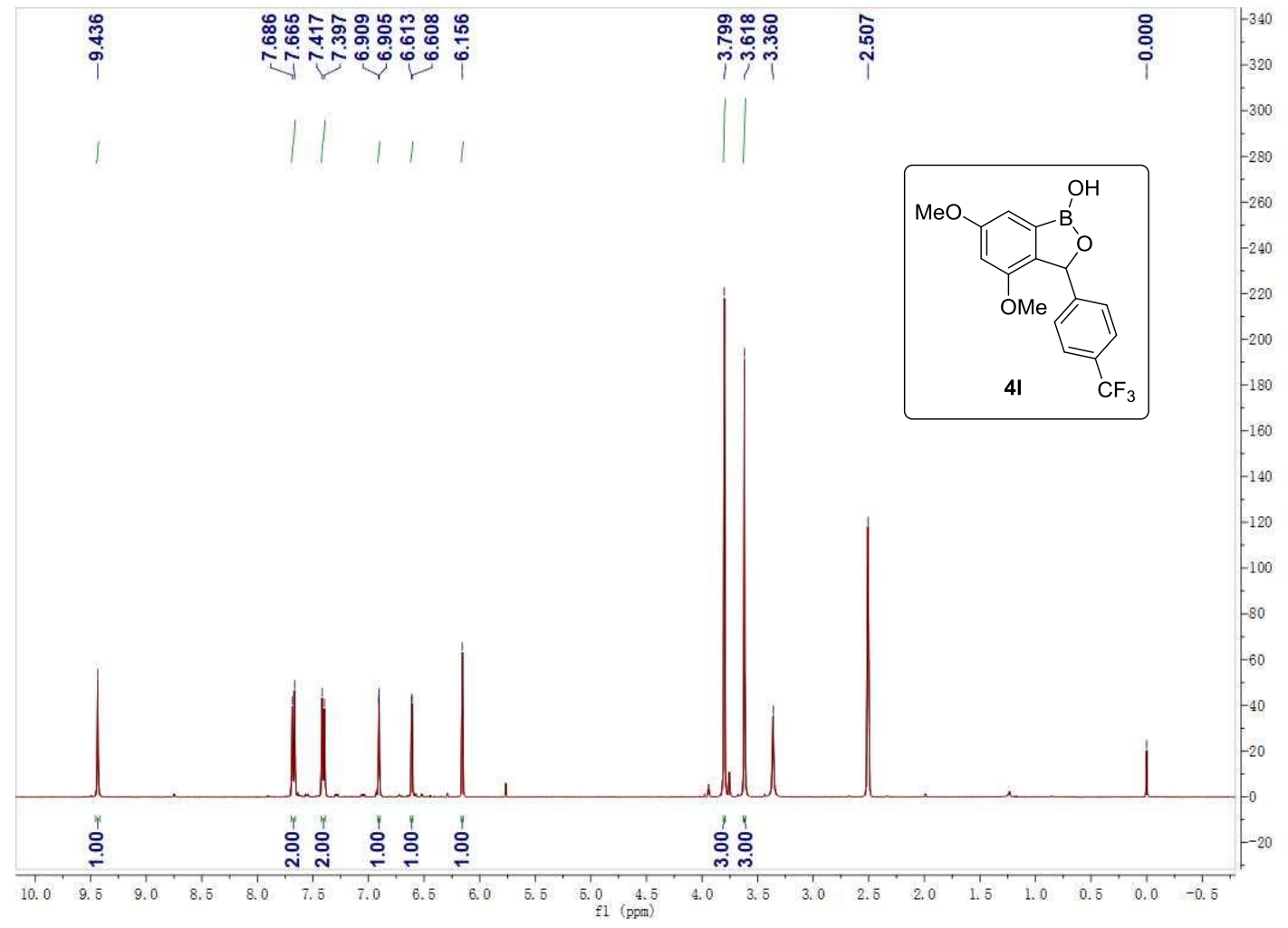

${ }^{13}$ C NMR (125 MHz, DMSO-d6)

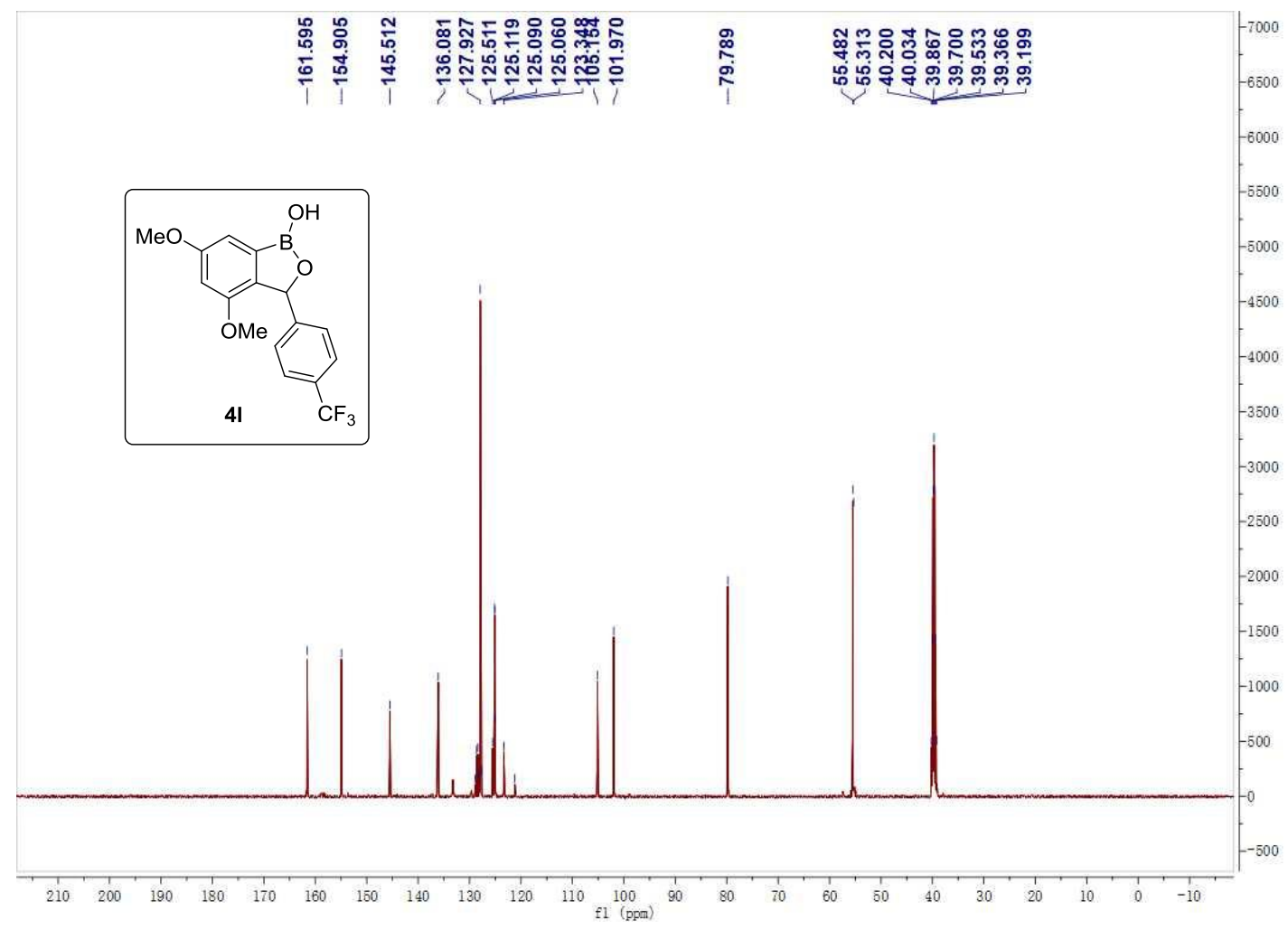


methyl 4-(1-hydroxy-4,6-dimethoxy-1,3-dihydrobenzo[c] [1,2] oxaborol-3-yl) benzoate (4m)

${ }^{1}$ H NMR (500 MHz, DMSO-d6)

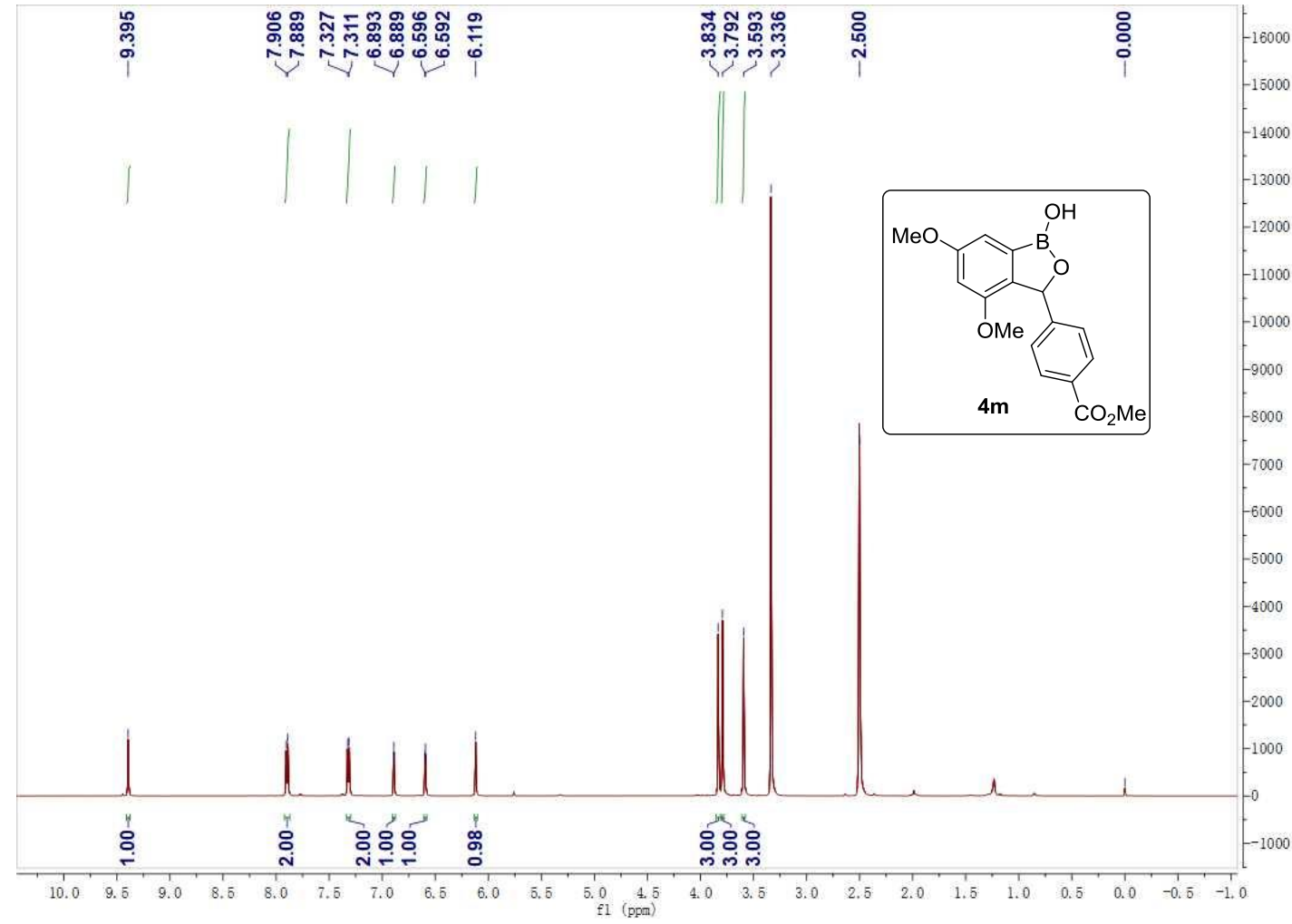

${ }^{13}$ C NMR (125 MHz, DMSO- $\left.d 6\right)$

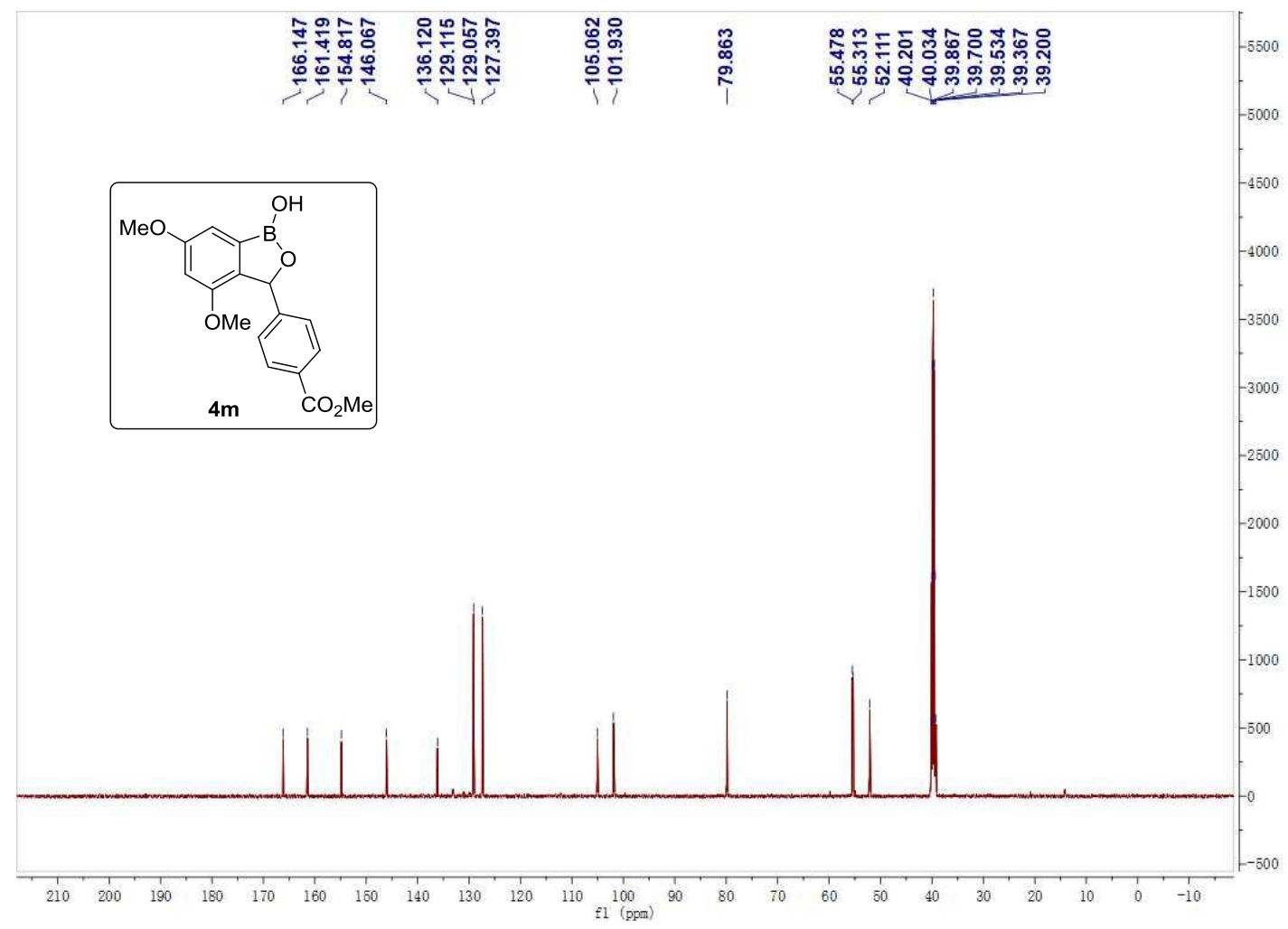


4-(1-hydroxy-4,6-dimethoxy-1,3-dihydrobenzo[c] [1,2] oxaborol-3-yl) benzonitrile (4n)

${ }^{1} \mathbf{H}$ NMR (400 MHz, DMSO-d6)

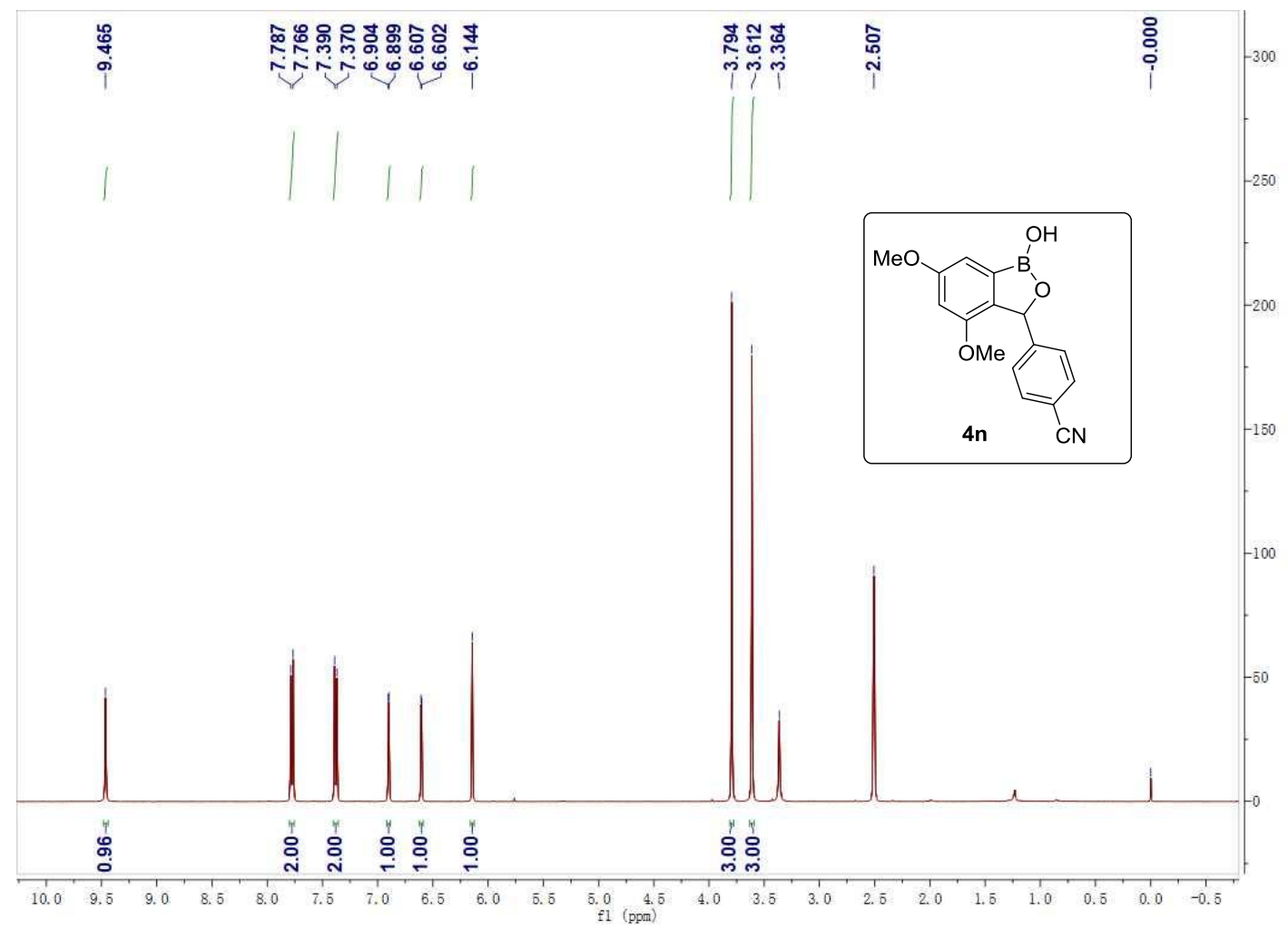

${ }^{13}$ C NMR (125 MHz, DMSO-d6)

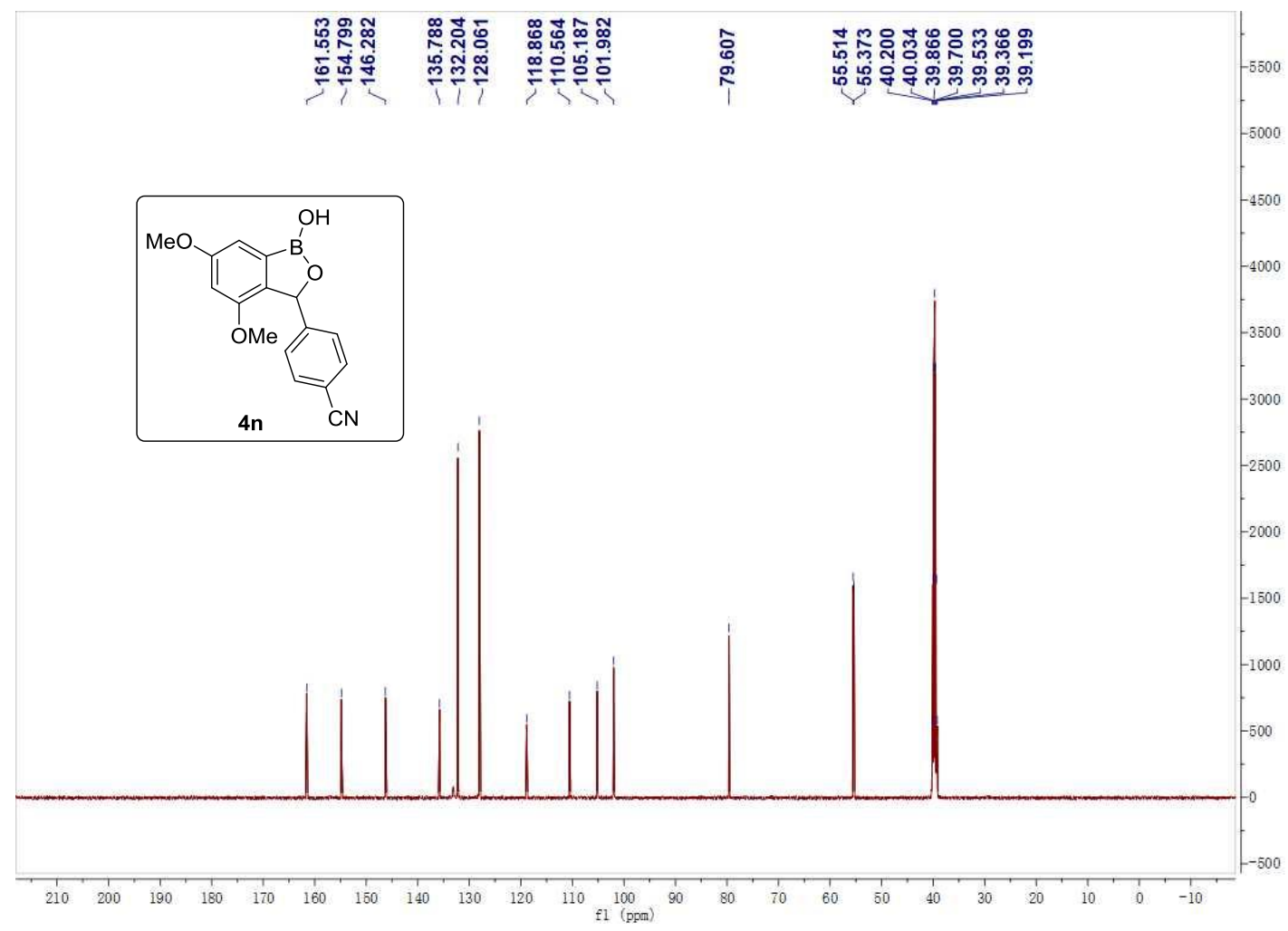


4,6-dimethoxy-3-(4-nitrophenyl) benzo[c] [1,2] oxaborol-1(3H)-ol (4o)

${ }^{1}$ H NMR (400 MHz, DMSO-d6)

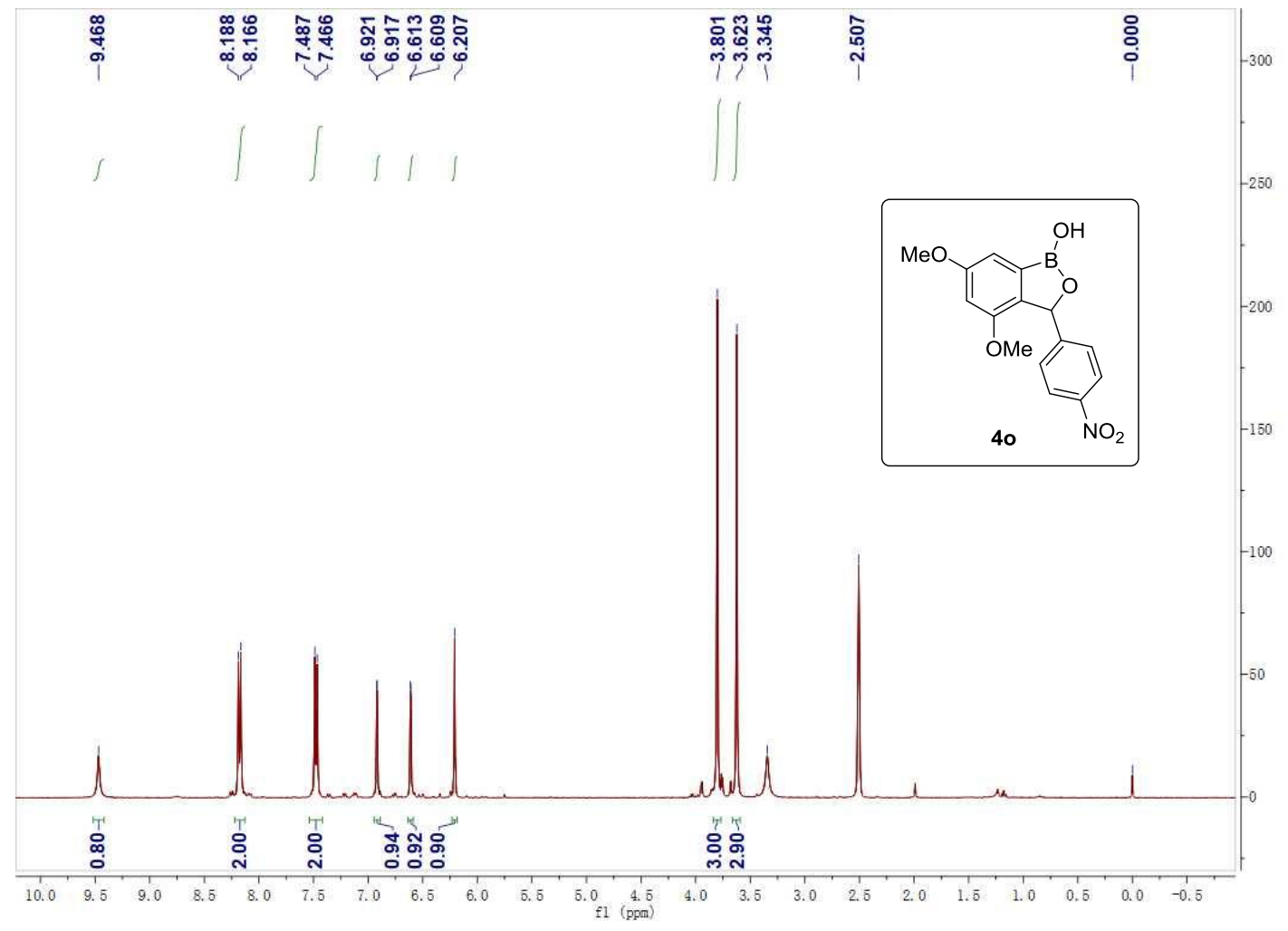

${ }^{13}$ C NMR (125 MHz, DMSO- $\left.d 6\right)$

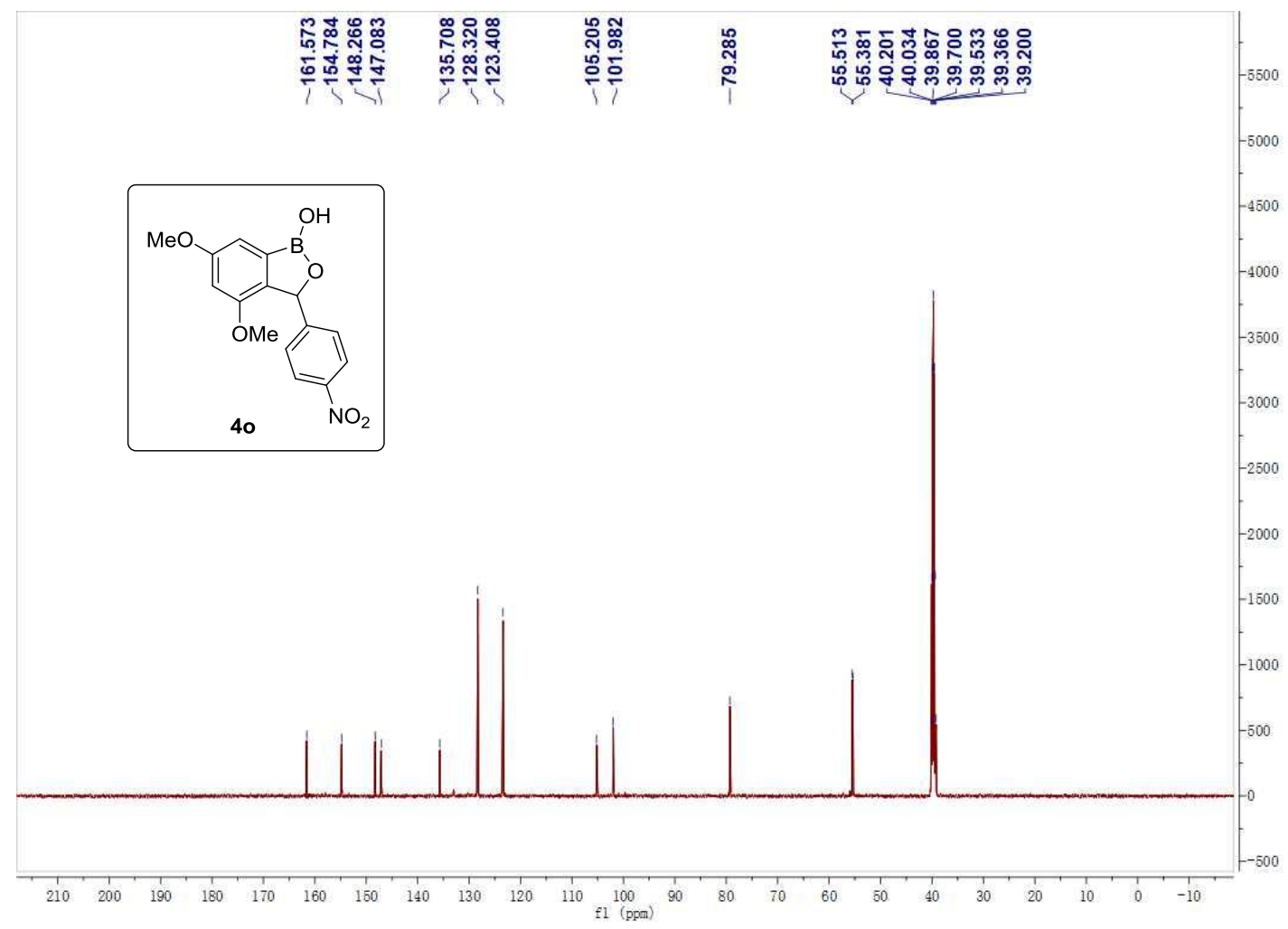


4,6-dimethoxy-3-(2-methoxyphenyl) benzo [c] [1,2] oxaborol-1(3H)-ol (4p)

${ }^{1} \mathbf{H}$ NMR (500 MHz, DMSO-d6)

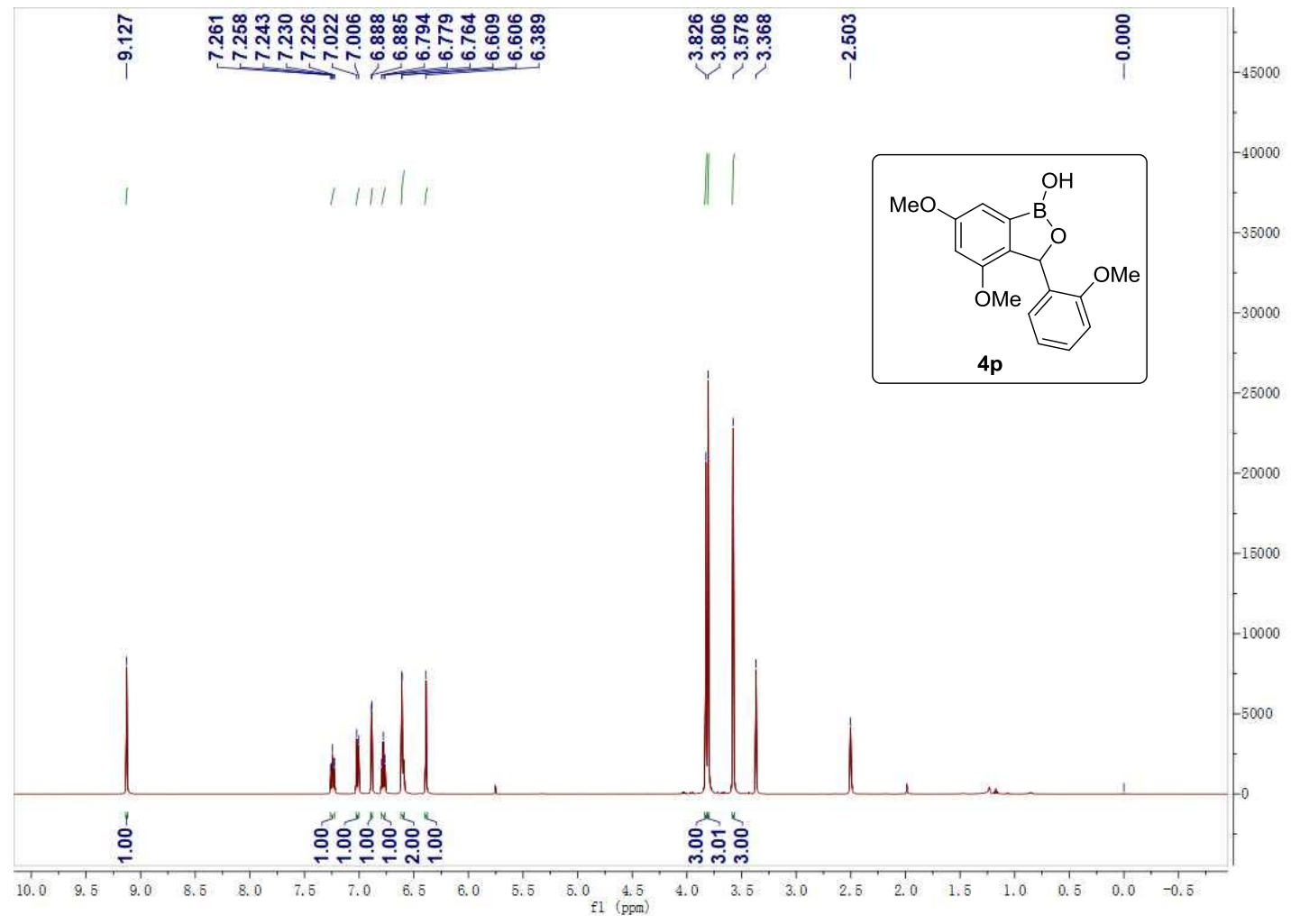

${ }^{13}$ C NMR (125 MHz, DMSO- $\left.d 6\right)$

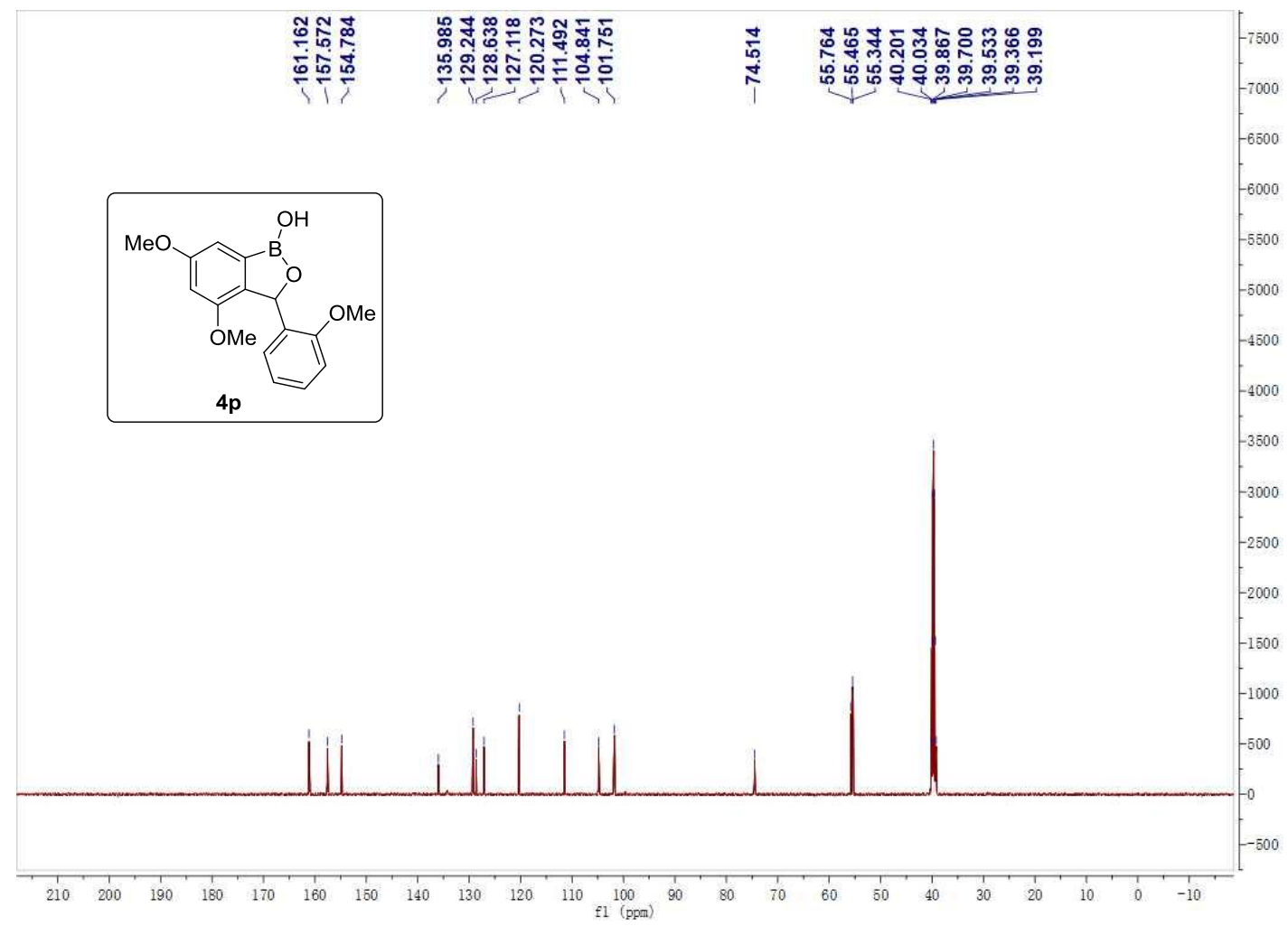


3-(2-fluorophenyl)-4,6-dimethoxybenzo[c] [1,2] oxaborol-1(3H)-ol (4q)

${ }^{1} \mathbf{H}$ NMR (500 MHz, DMSO-d6)

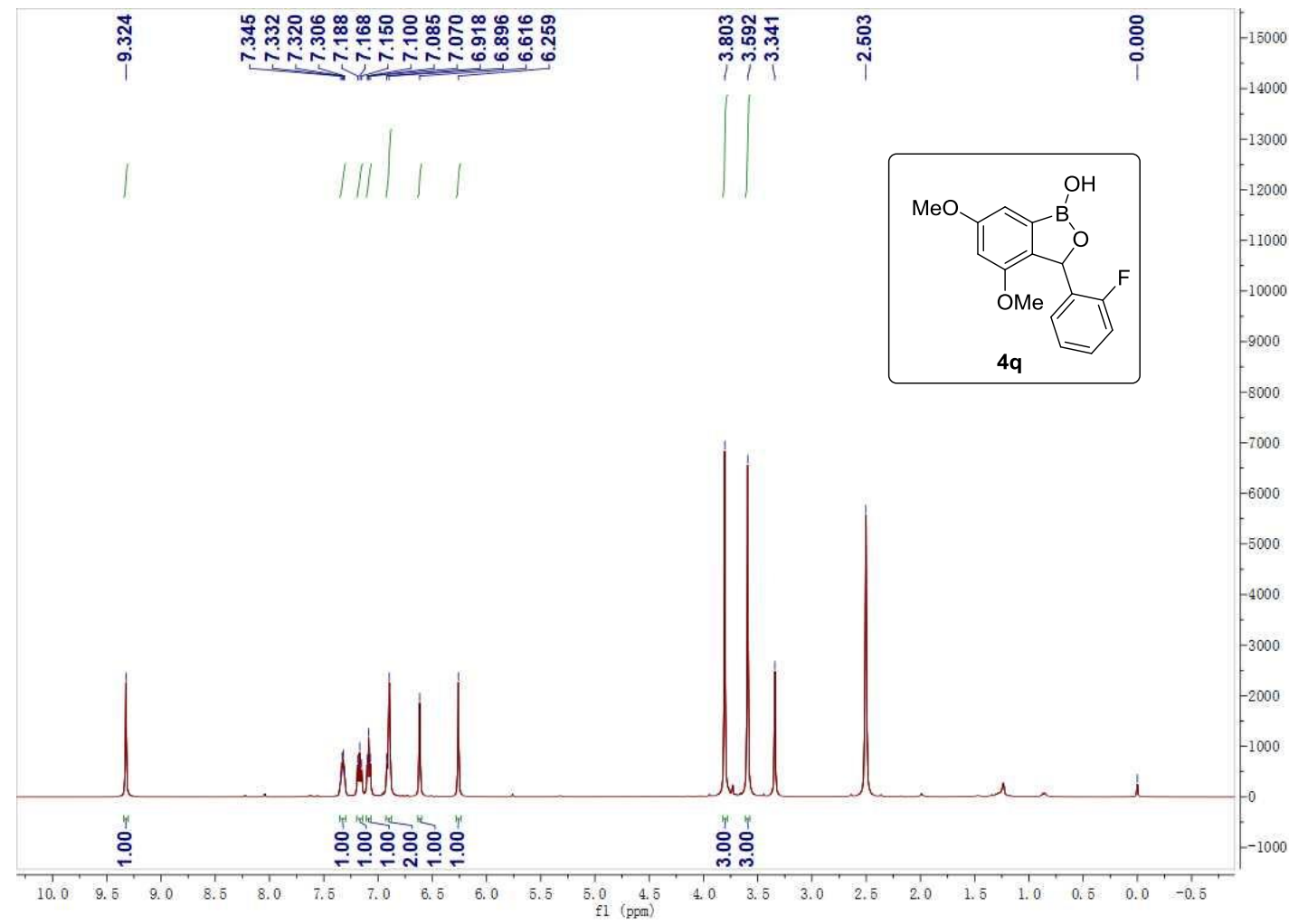

${ }^{13}$ C NMR (125 MHz, DMSO- $\left.d 6\right)$

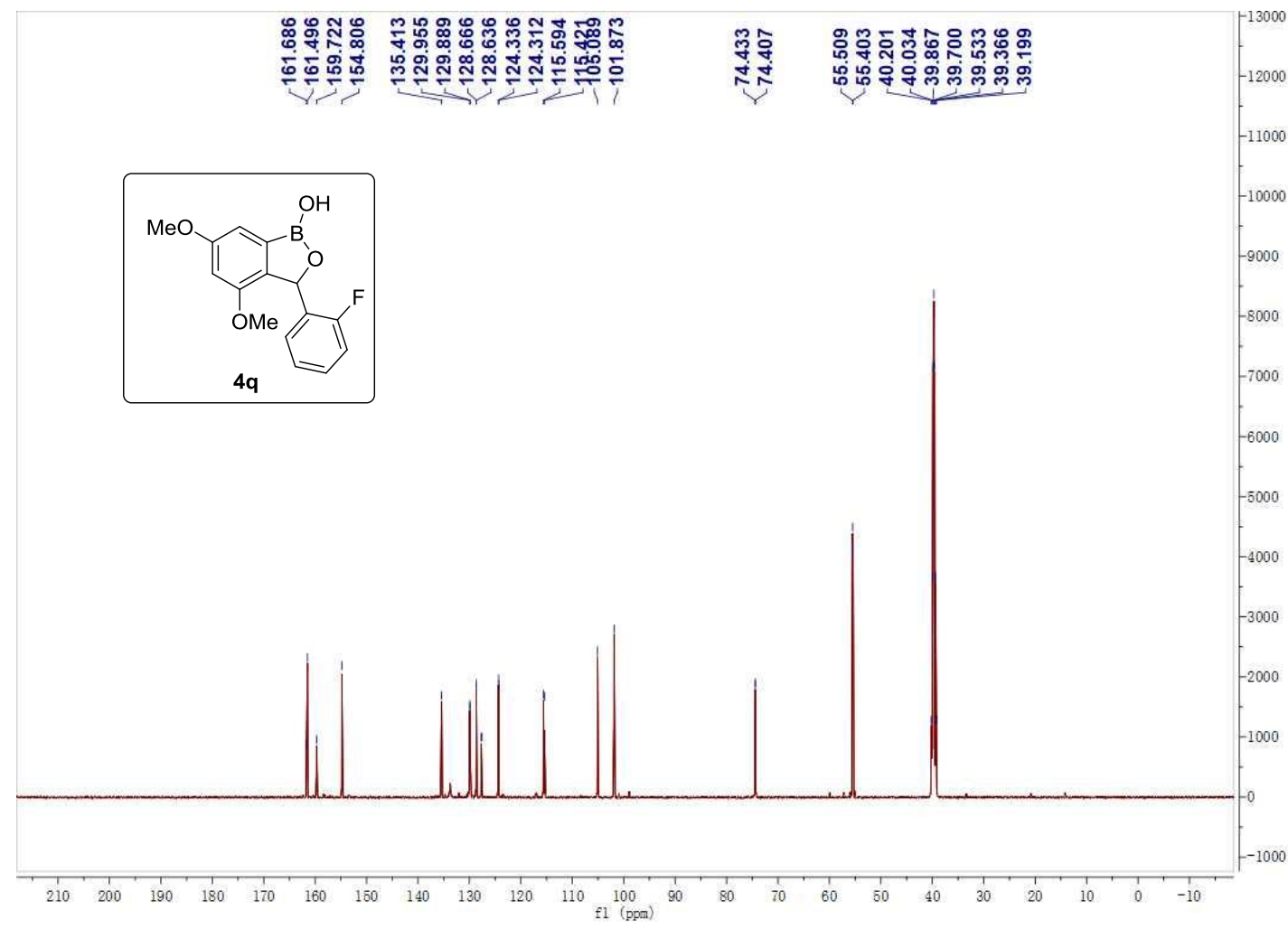


4,6-dimethoxy-3-(2-nitrophenyl) benzo[c] [1,2] oxaborol-(3H)-ol (4r)

${ }^{1}$ H NMR (400 MHz, DMSO-d6)

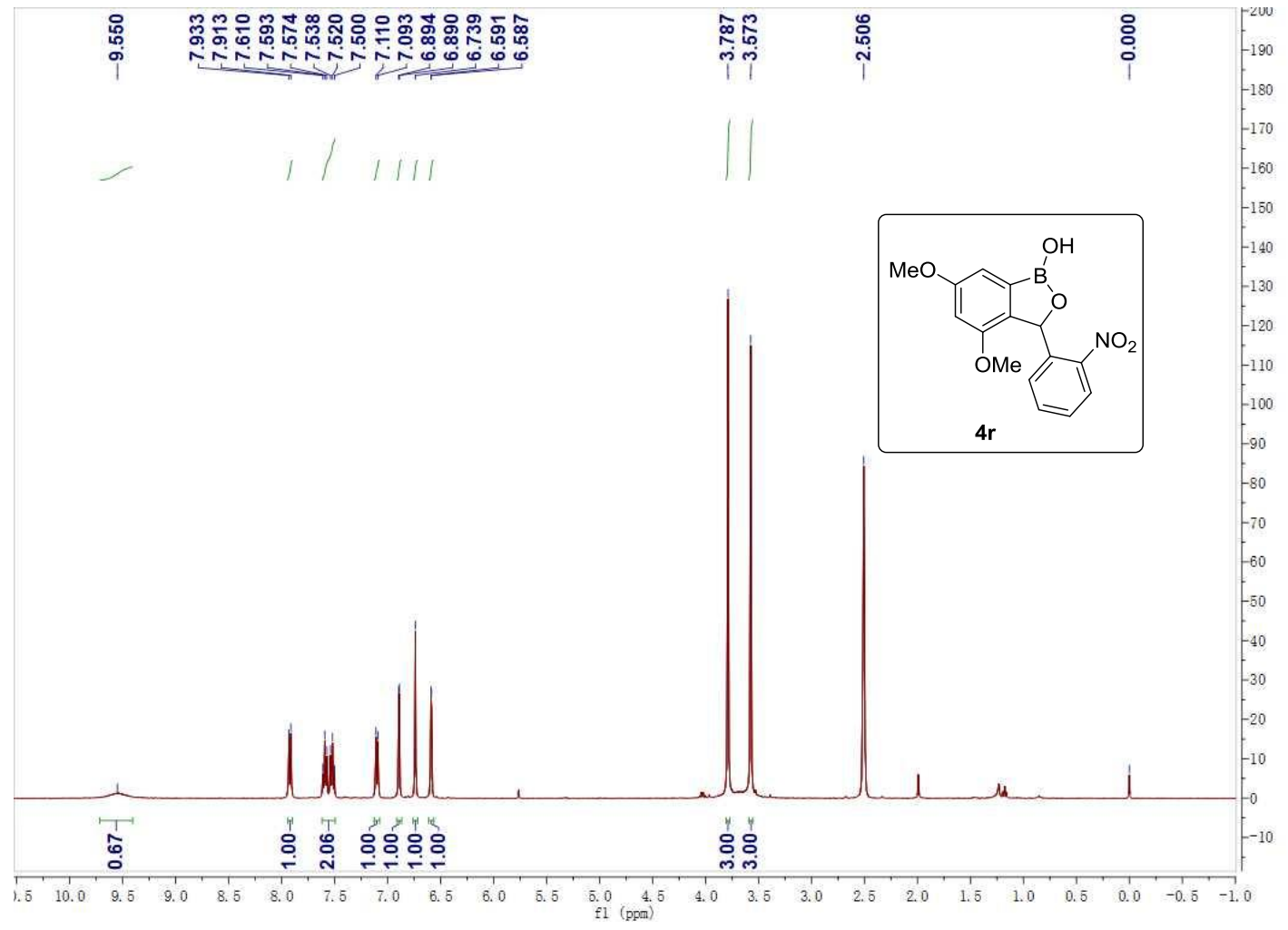

${ }^{13}$ C NMR (125 MHz, DMSO-d6)

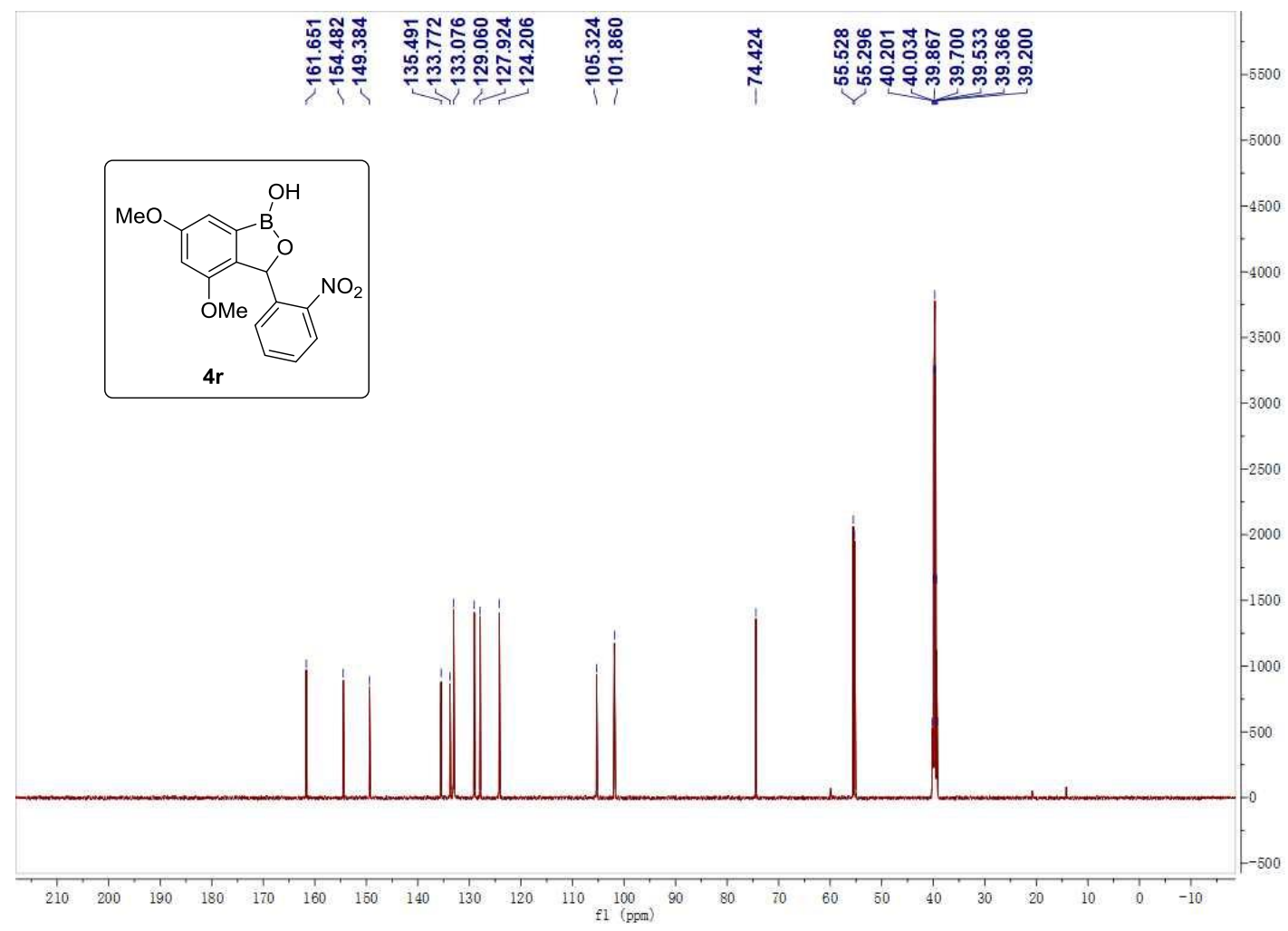


4,6-dimethoxy-3,3-dimethylbenzo[c] [1,2] oxaborol-1(3H)-ol (5a)

${ }^{1}$ H NMR (400 MHz, DMSO-d6)

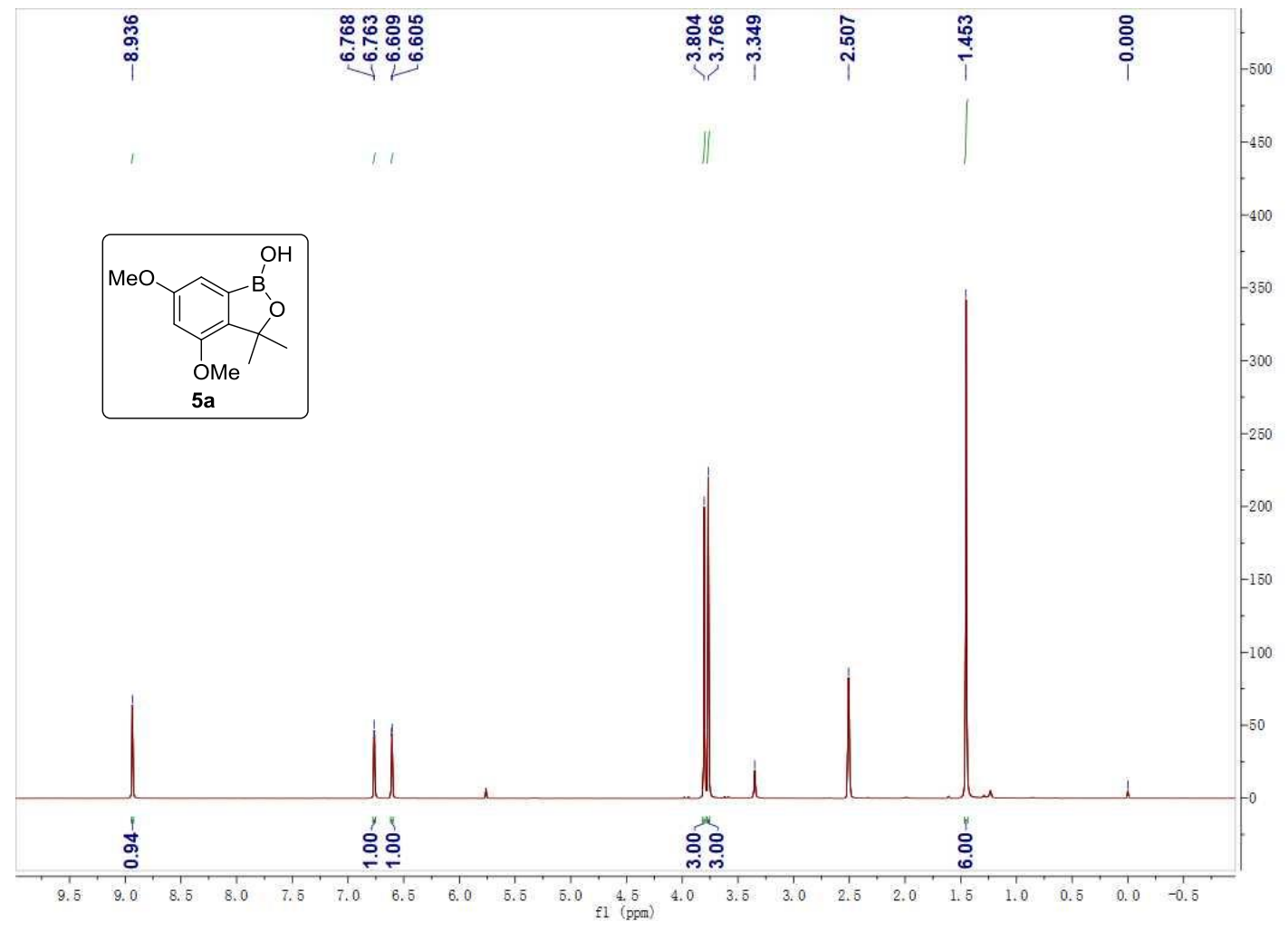

${ }^{13}$ C NMR (125 MHz, DMSO-d6)

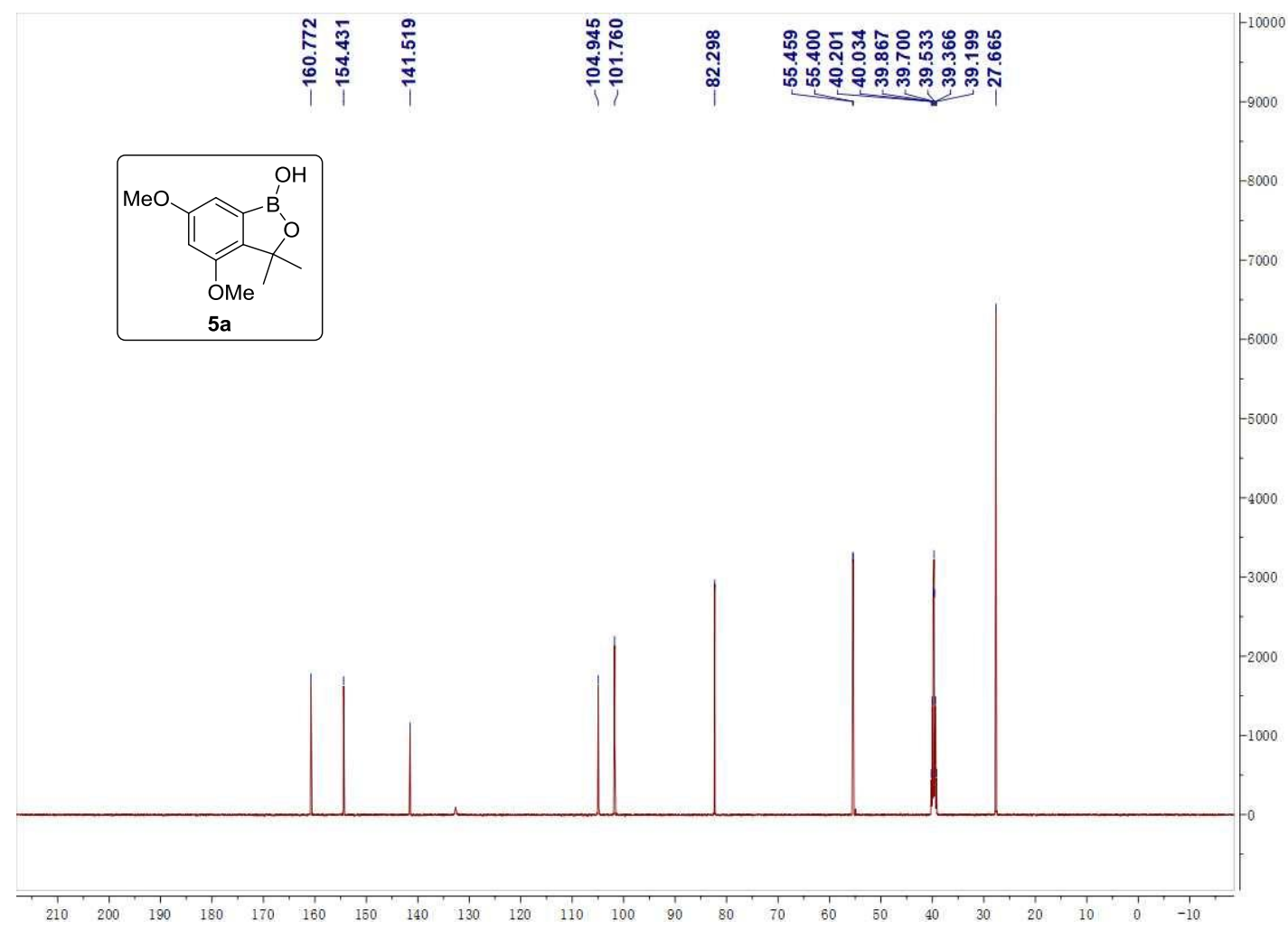


4,6-dimethoxy-1H-spiro[benzo[c] [1,2] oxaborole-3,1'-cyclobutan]-1-ol (5b)

${ }^{1}$ H NMR (400 MHz, DMSO-d6)

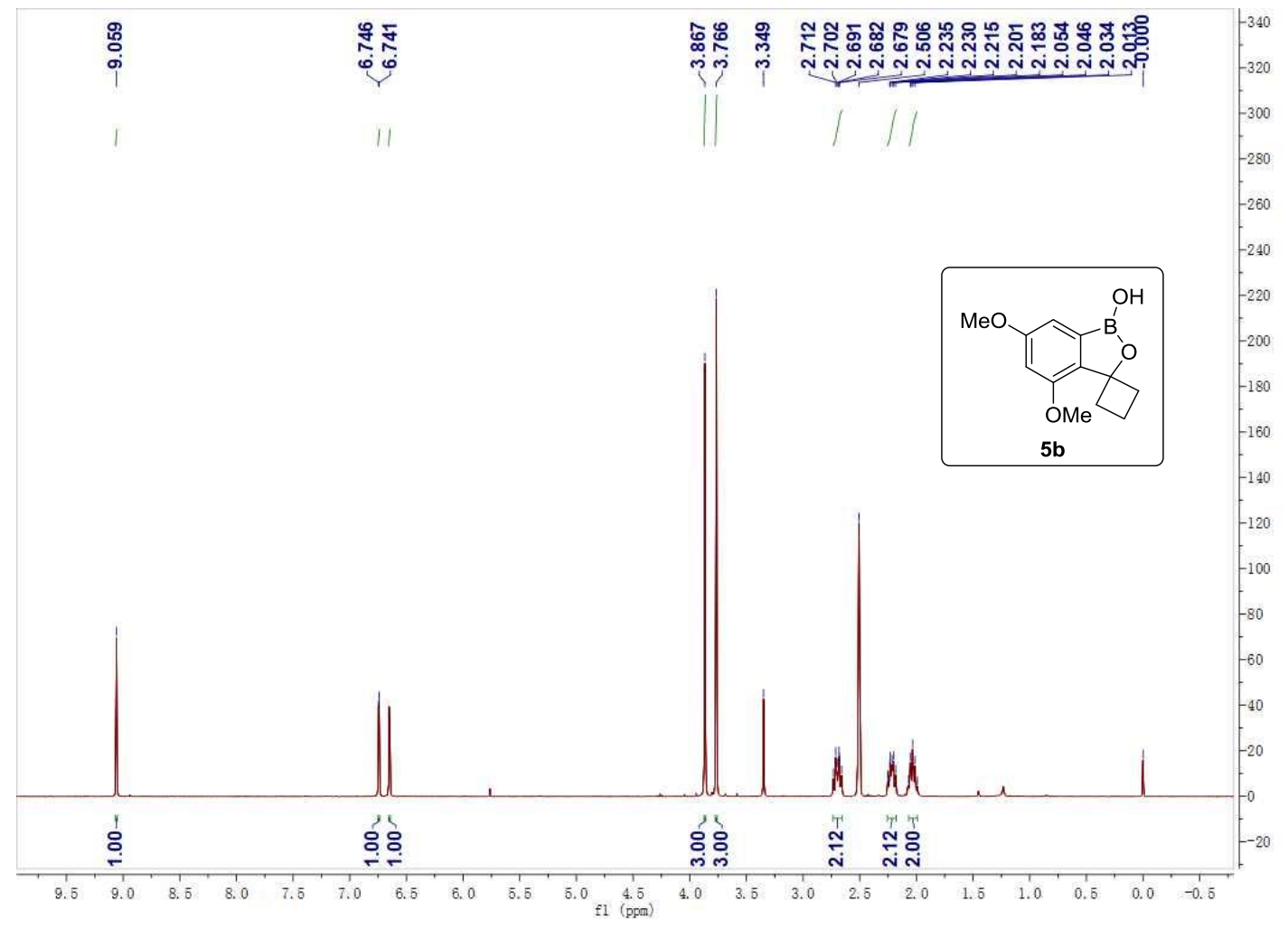

${ }^{13}$ C NMR (125 MHz, DMSO- $\left.d 6\right)$

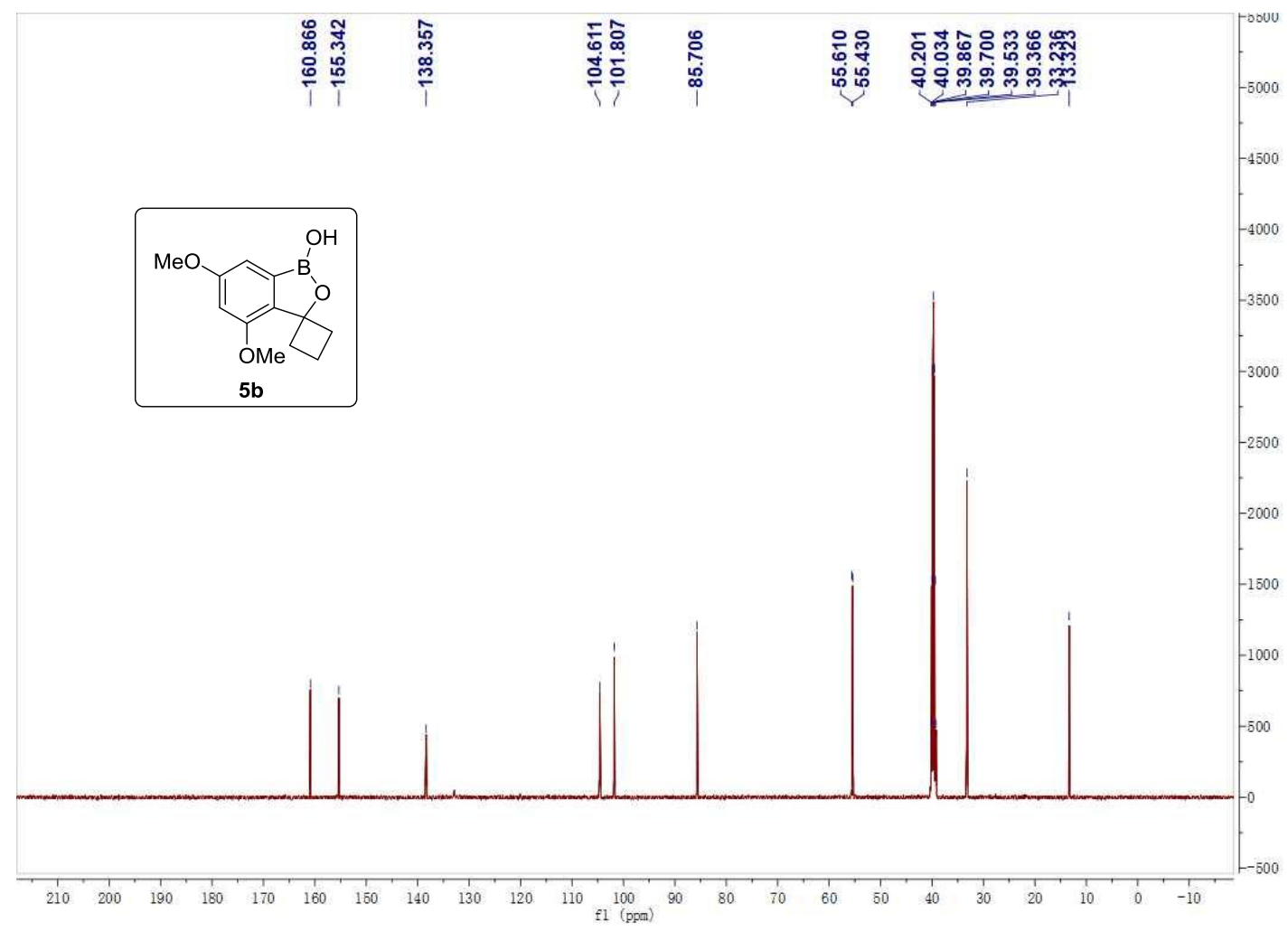


4,6-dimethoxy-1H-spiro[benzo[c] [1,2] oxaborole-3,1'-cyclopentan]-1-ol (5c)

${ }^{1}$ H NMR (500 MHz, DMSO-d6)

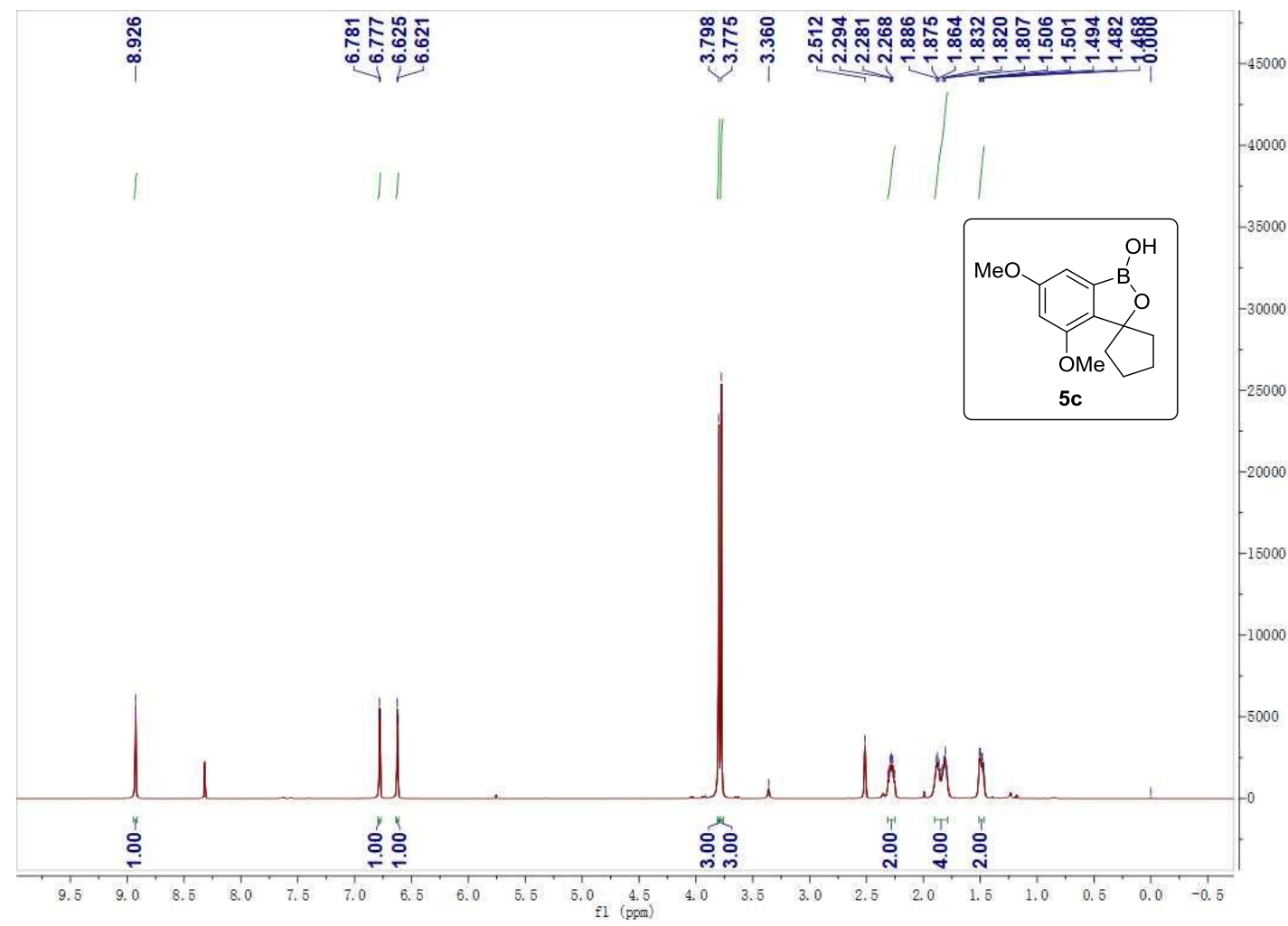

${ }^{13}$ C NMR (125 MHz, DMSO-d6)

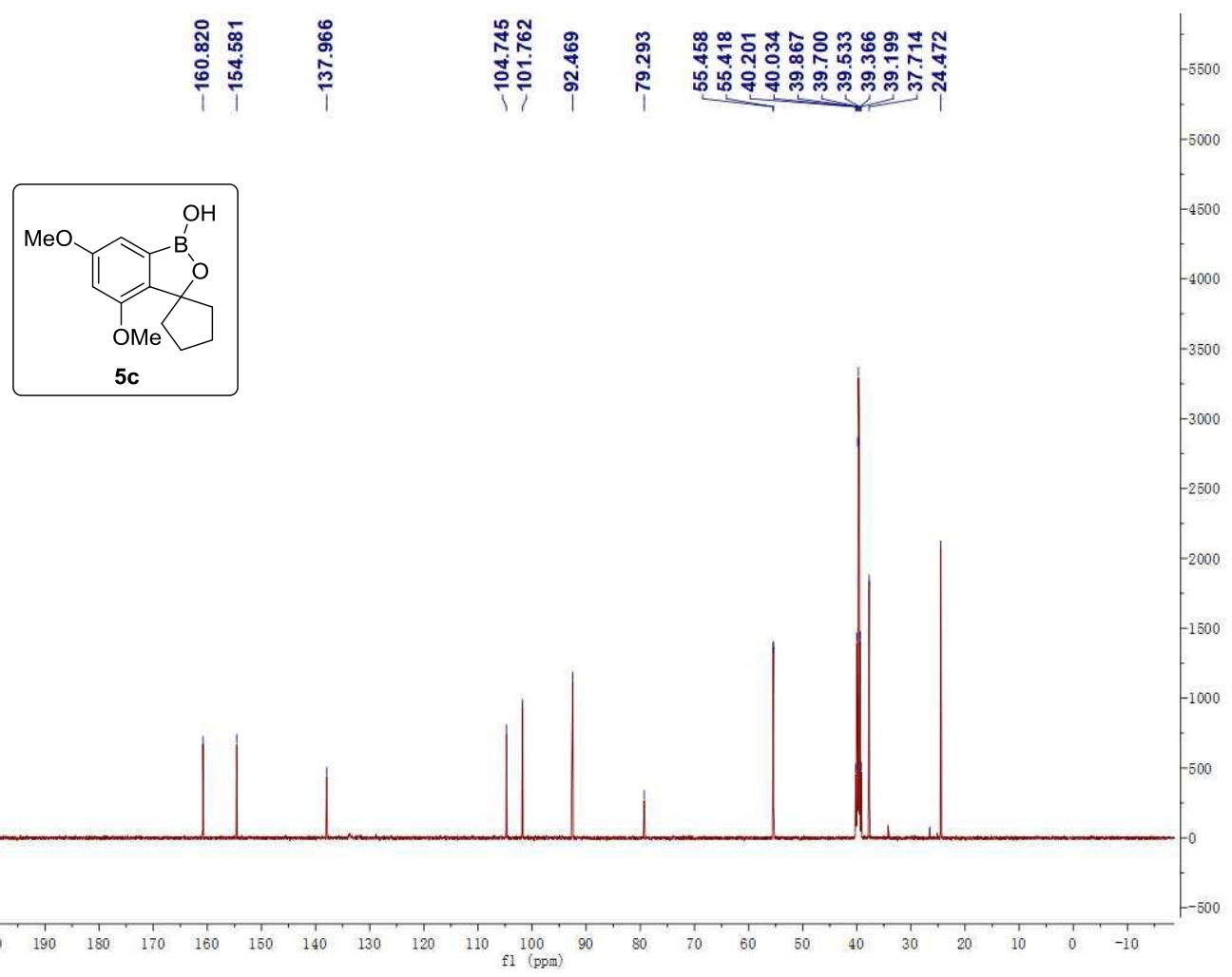


4,6-dimethoxy-1H-spiro[benzo[c] [1,2] oxaborole-3,1'-cyclohexan]-1-ol (5d)

${ }^{1} \mathbf{H}$ NMR (400 MHz, DMSO-d6)

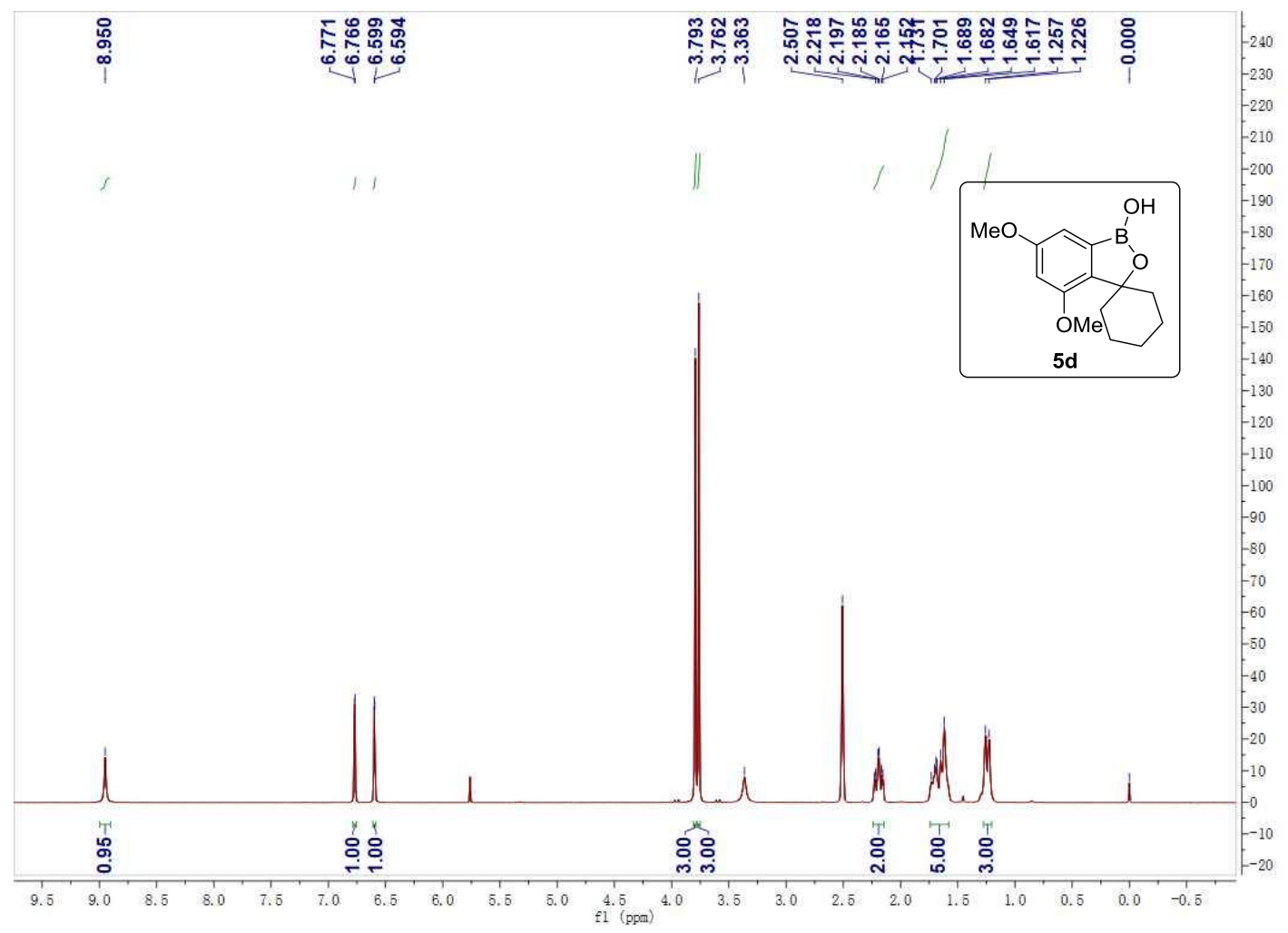

${ }^{13}$ C NMR (125 MHz, DMSO-d6)

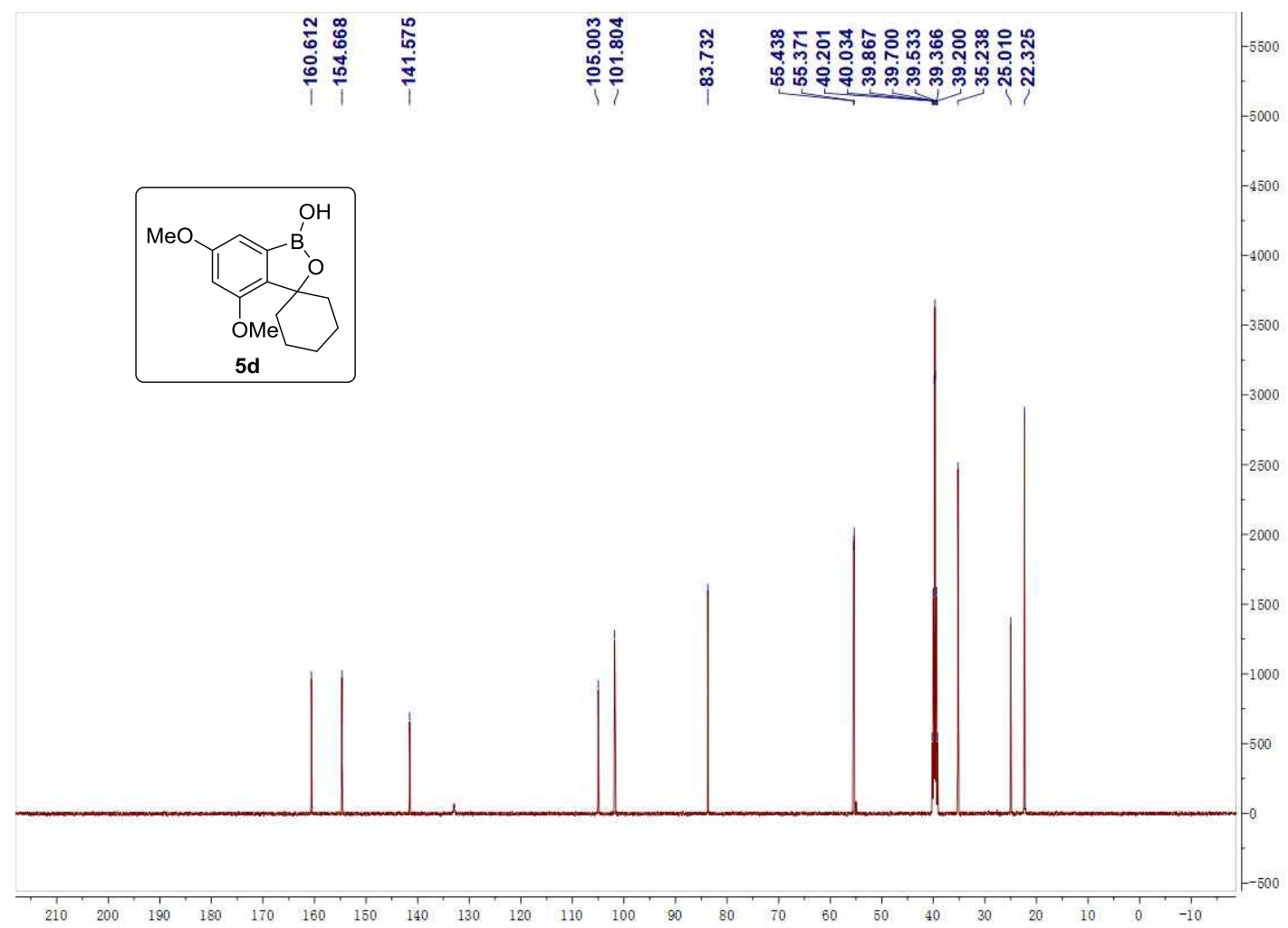


methyl 1-hydroxy-4,6-dimethoxy-3-methyl-1,3-dihydrobenzo[c] [1,2] oxaborole-3- carboxylate (5e)

${ }^{1}$ H NMR (500 MHz, DMSO-d6)

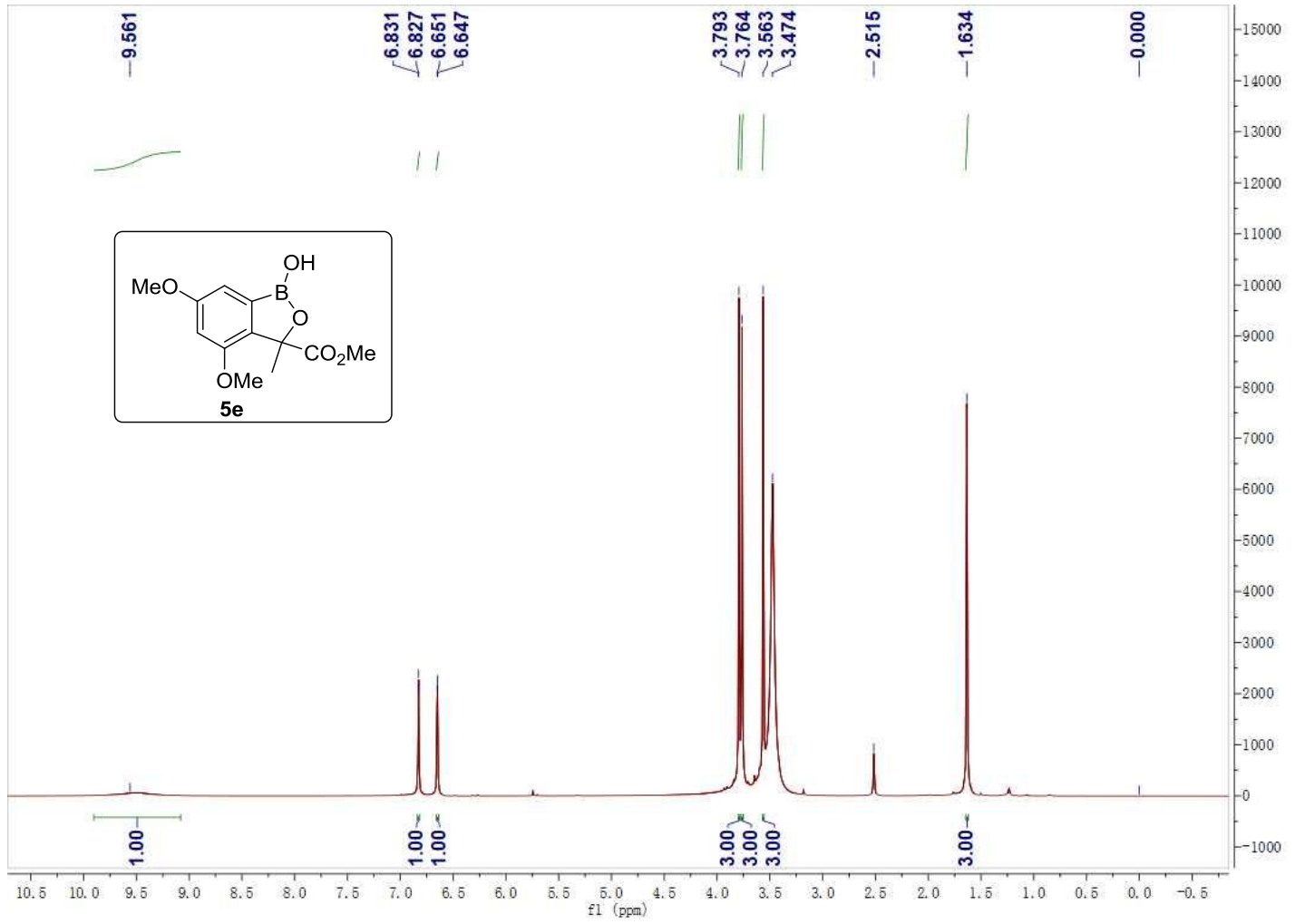

${ }^{13}$ C NMR (125 MHz, DMSO- $\left.d 6\right)$

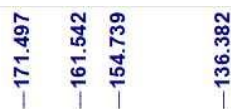

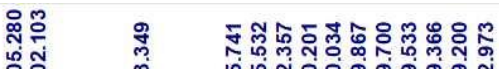

นำ ํํㅇㅇำ

$-4500$
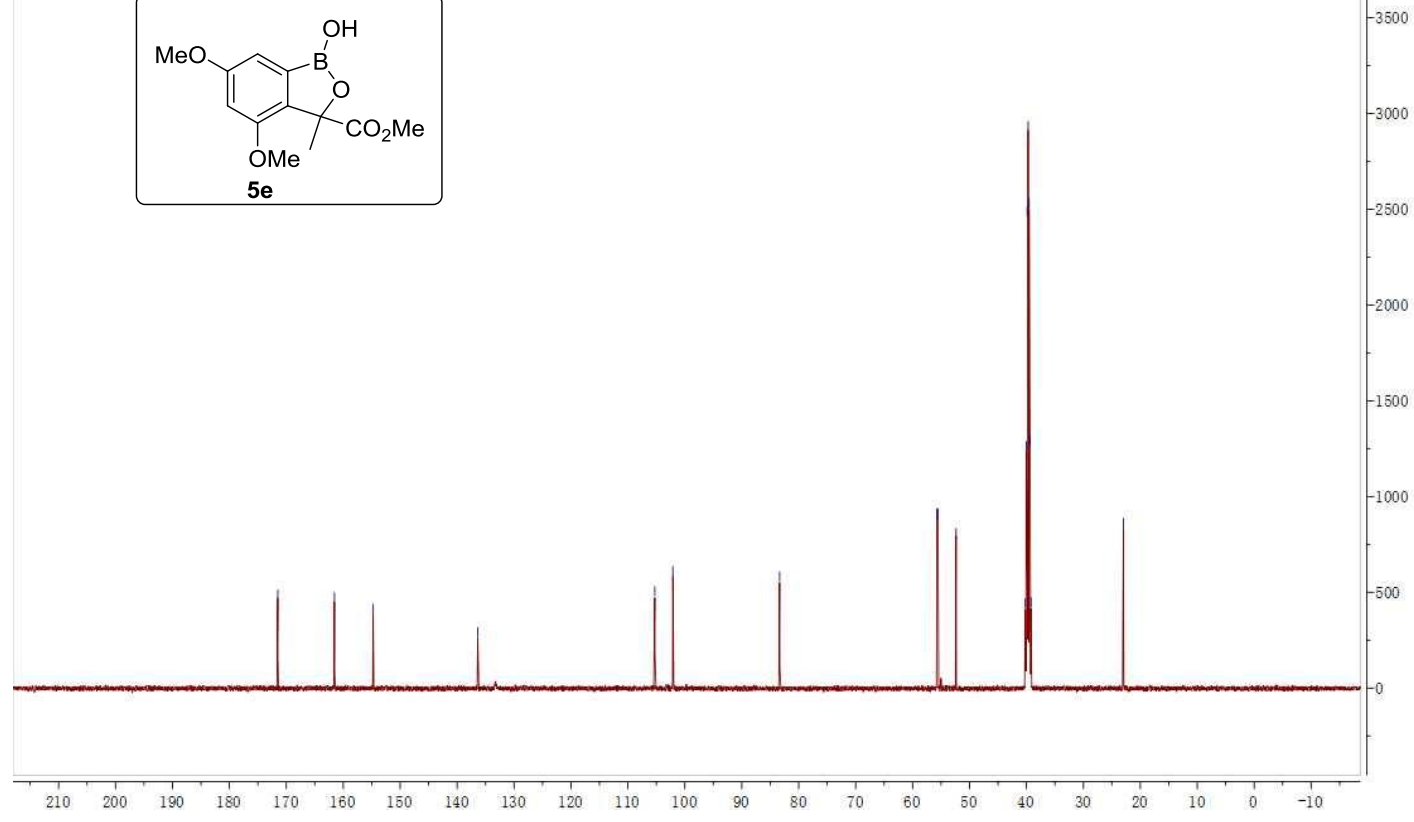
methyl 1-hydroxy-4,6-dimethoxy-3-methyl-1,3-dihydrobenzo[c] [1,2] oxaborole-3- carboxylate (5f)

${ }^{1}$ H NMR (400 MHz, DMSO-d6)

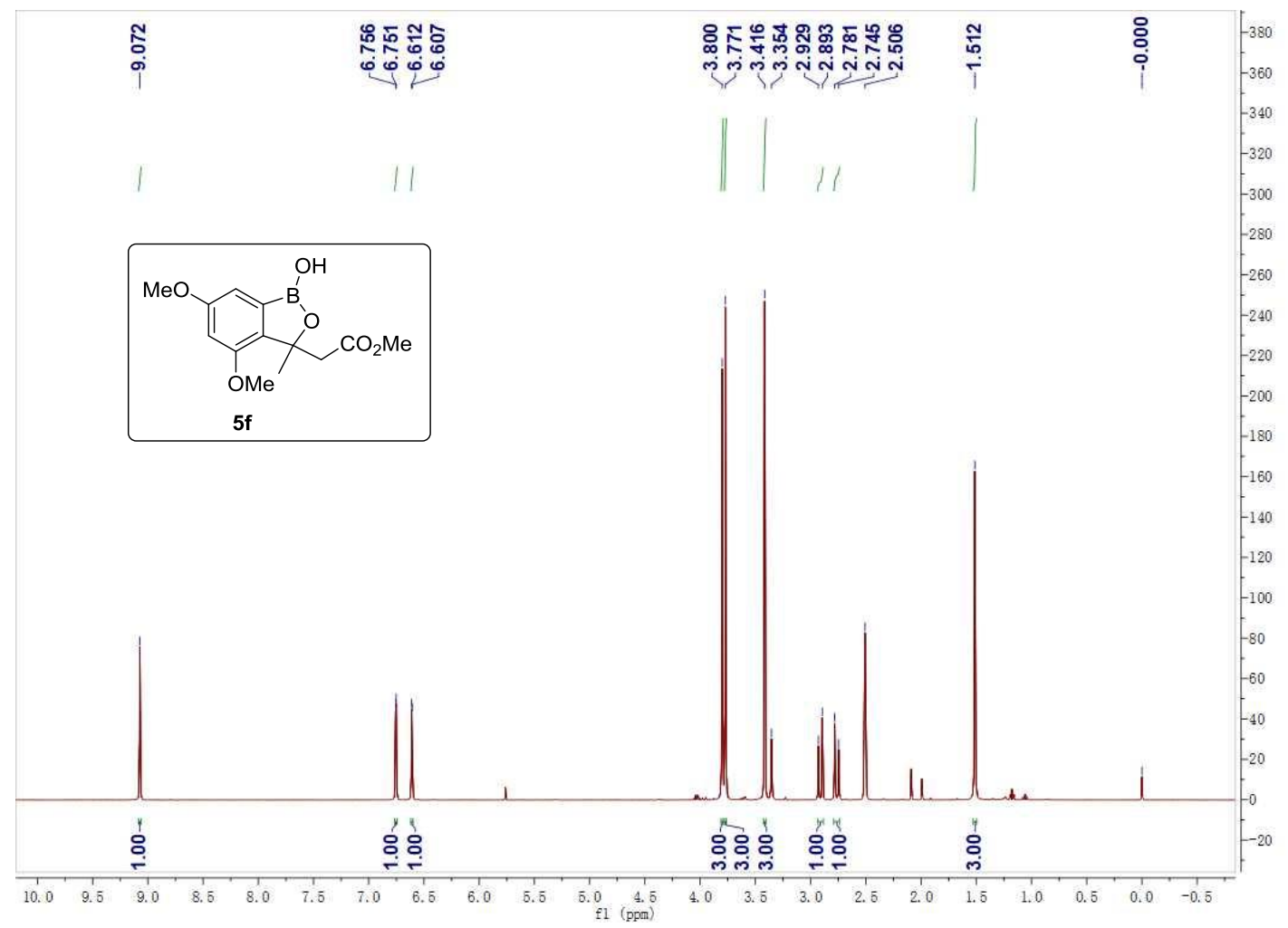

${ }^{13}$ C NMR (125 MHz, DMSO- $\left.d 6\right)$

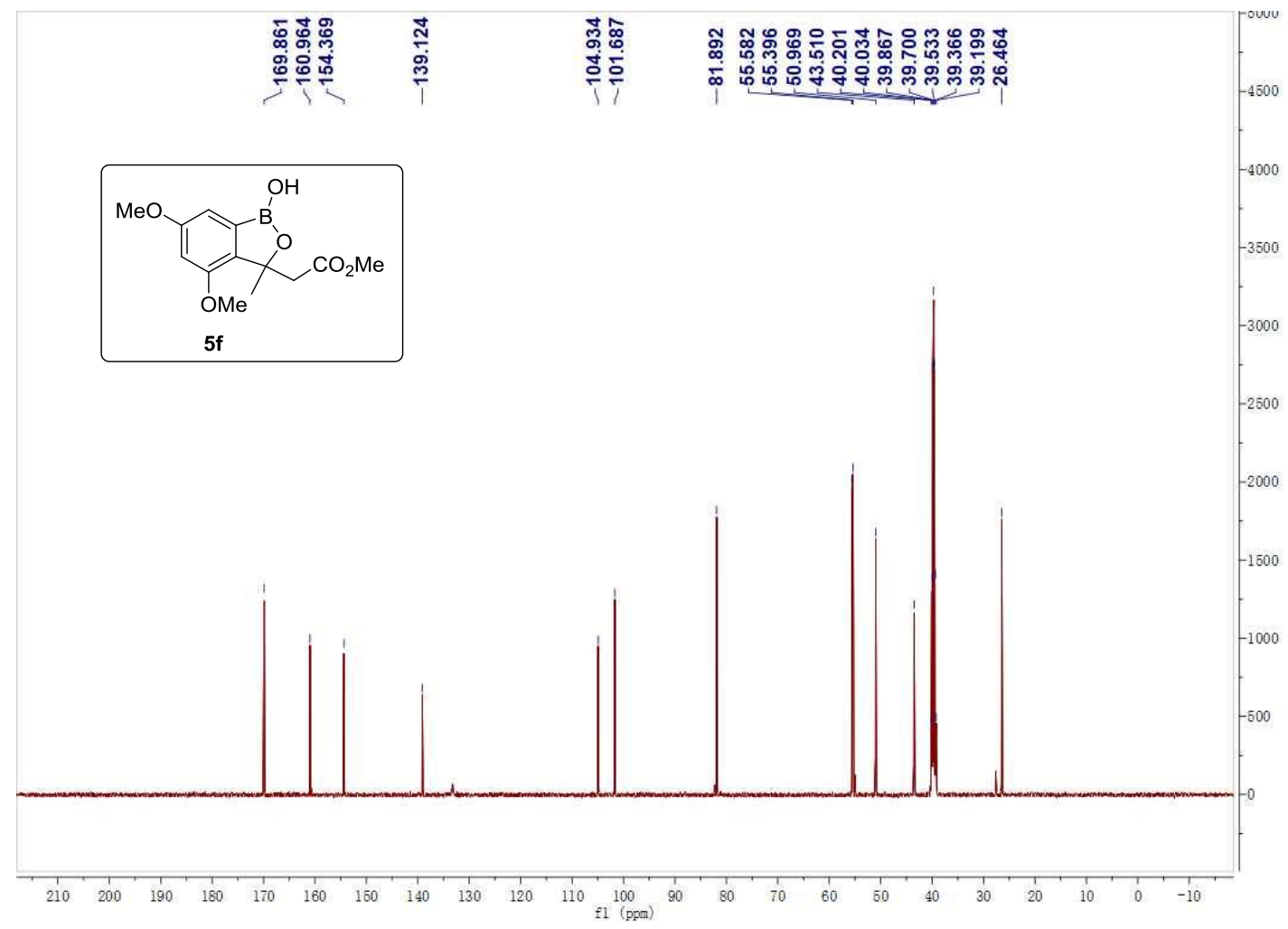


4,6-dimethoxy-3-phenyl-3-(trifluoromethyl) benzo[c] [1,2] oxaborol-1(3H)-ol (5h)

${ }^{1}$ H NMR (400 MHz, DMSO-d6)

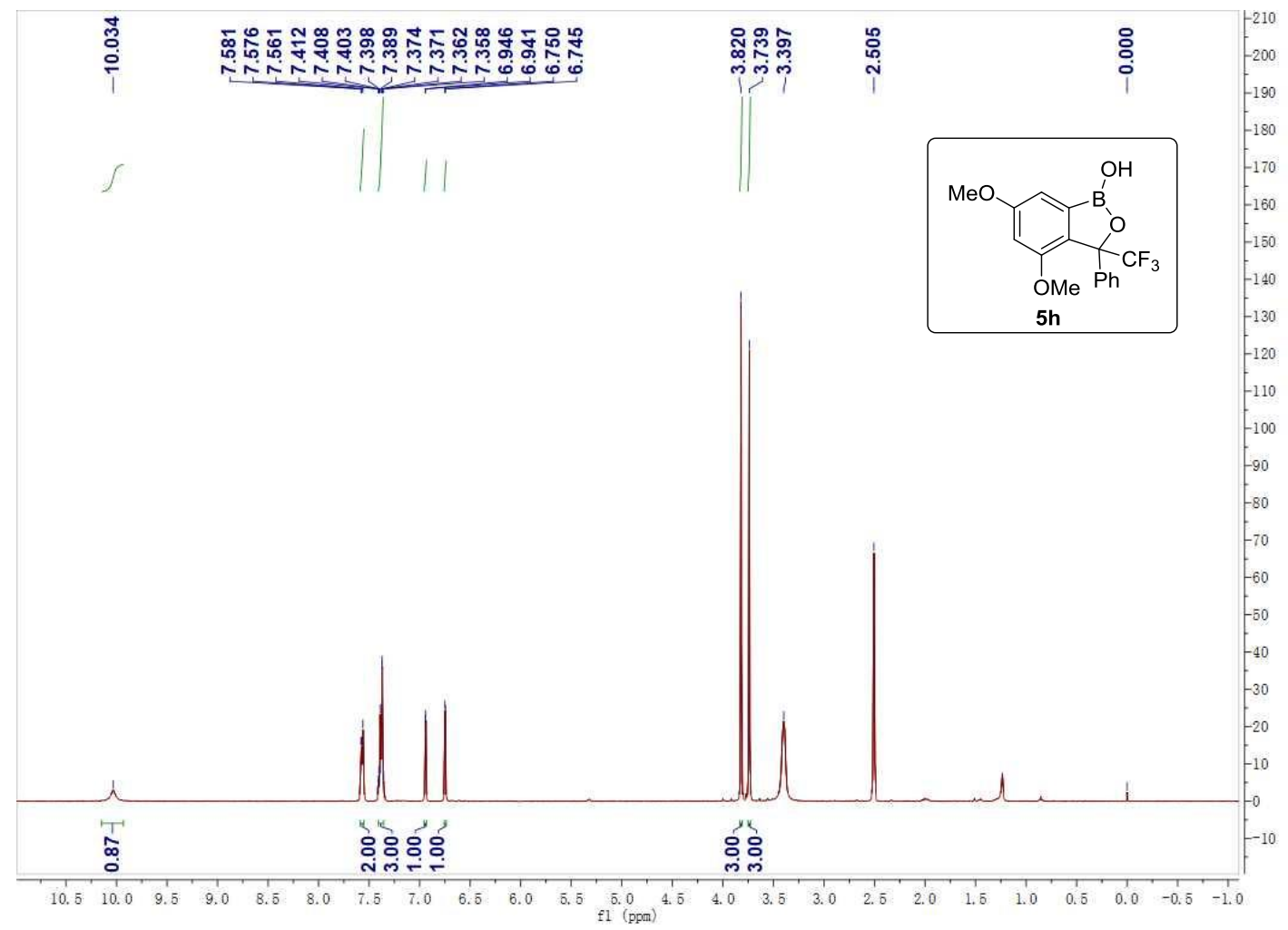

${ }^{13}$ C NMR (125 MHz, DMSO- $\left.d 6\right)$

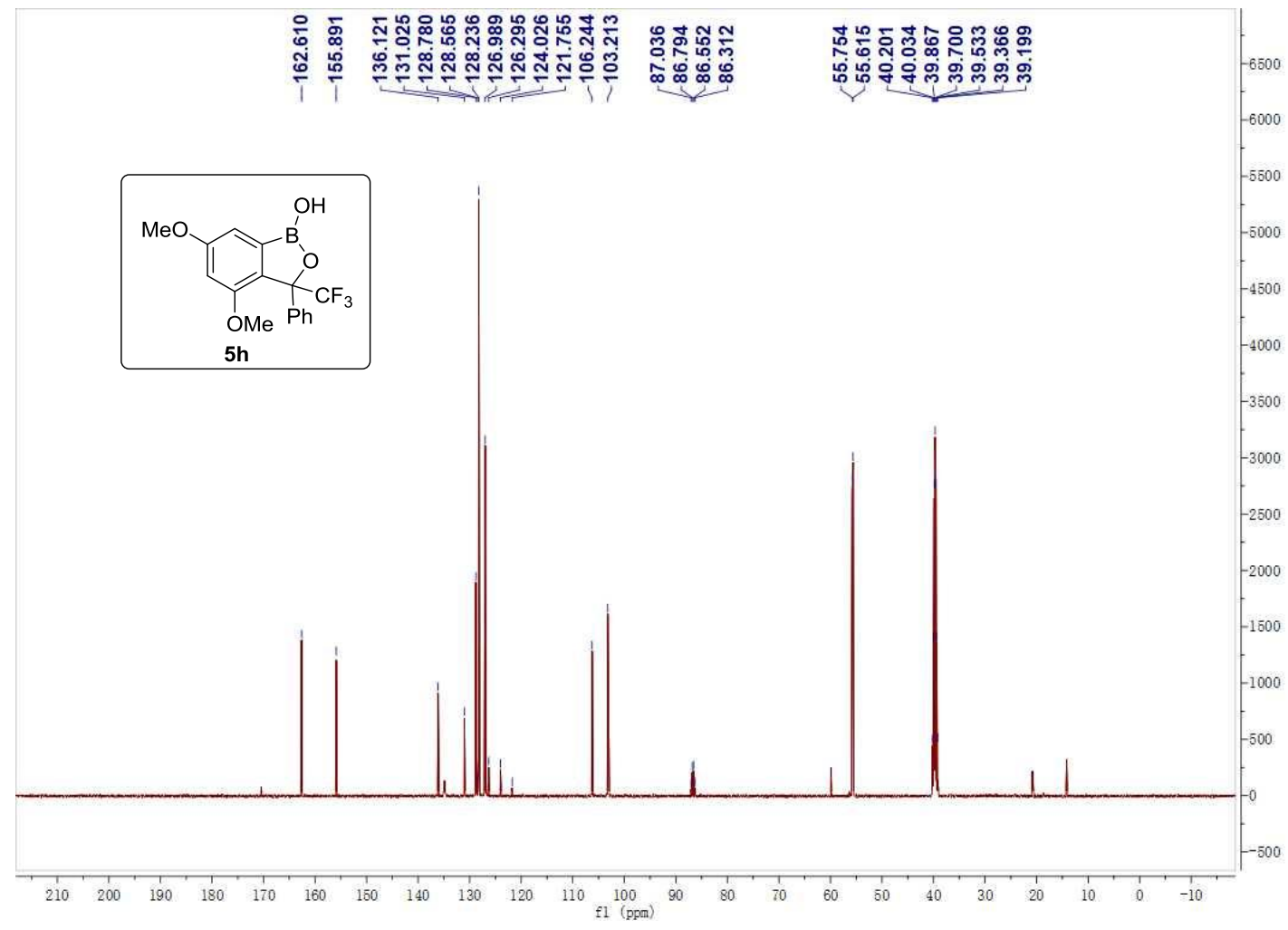


methyl 1-hydroxy-4,6-dimethoxy-3-phenyl-1,3-dihydrobenzo[c] [1,2] oxaborole-3-carboxylate (5i)

${ }^{1}$ H NMR (400 MHz, DMSO-d6)

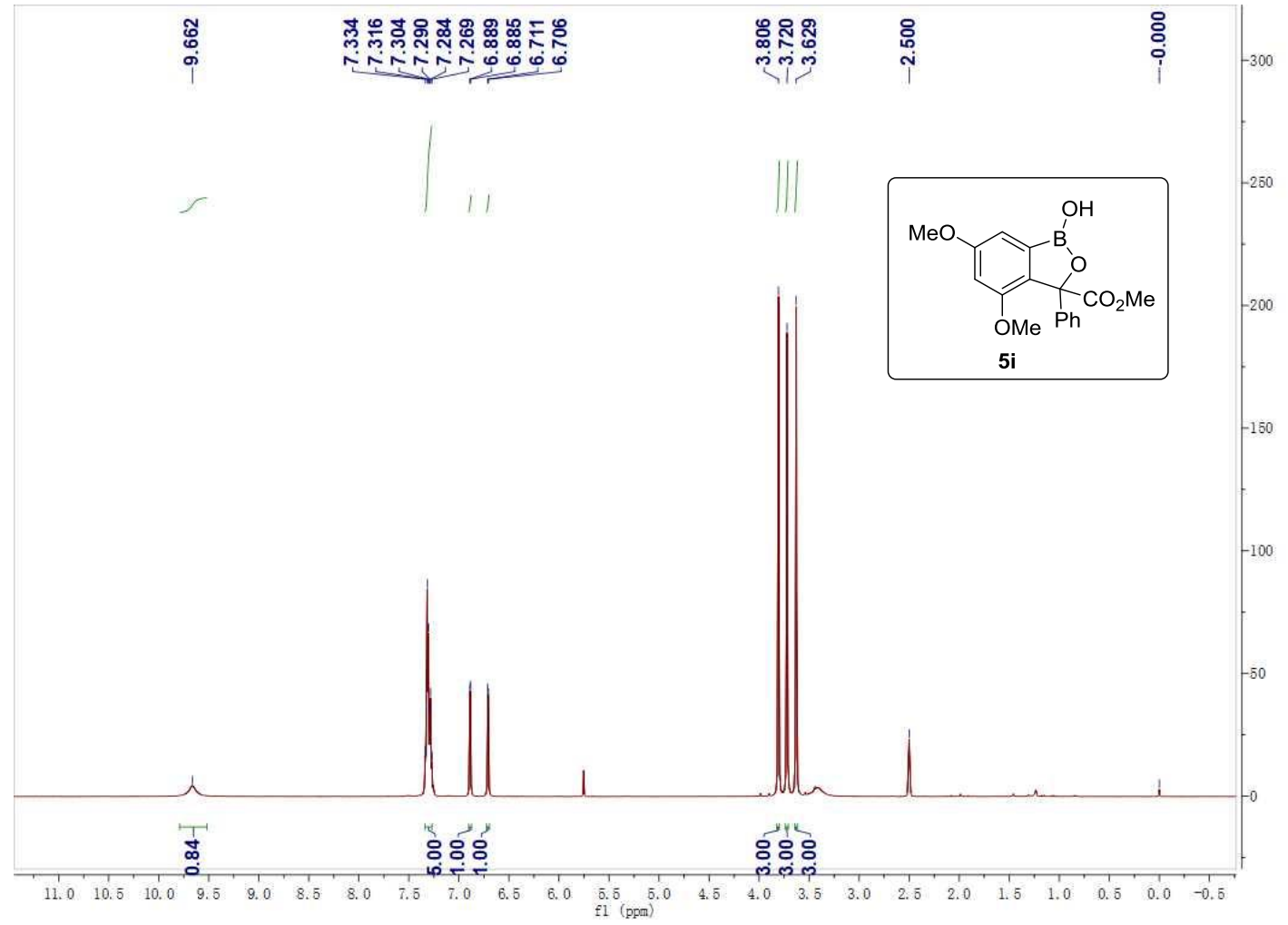

${ }^{13}$ C NMR (125 MHz, DMSO- $\left.d 6\right)$

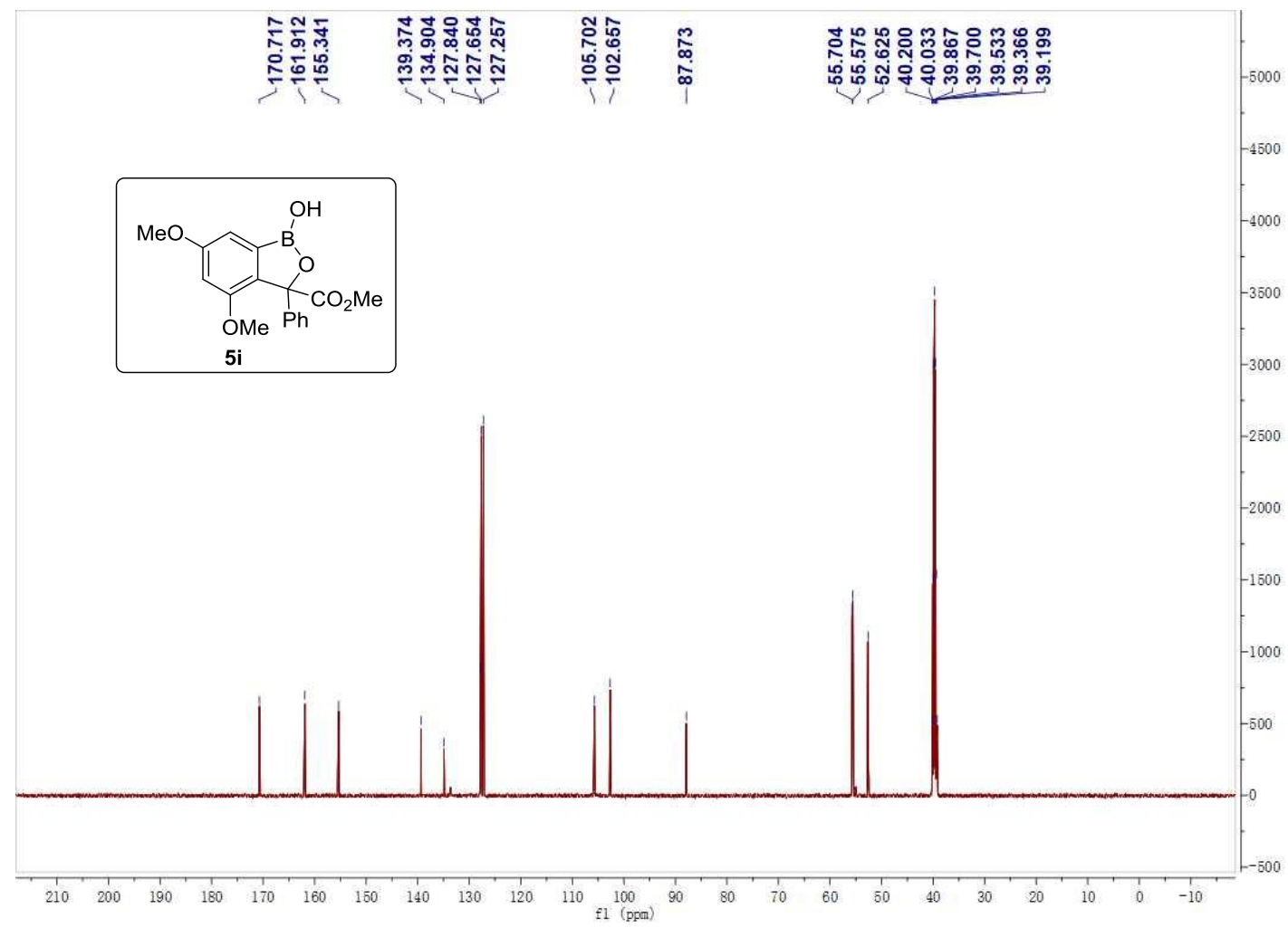


4,6-dimethoxy-3-methyl-3-(4-nitrophenyl) benzo[c] [1,2] oxaborol-1(3H)-ol (5j)

${ }^{1}$ H NMR (400 MHz, DMSO- $d 6$ )

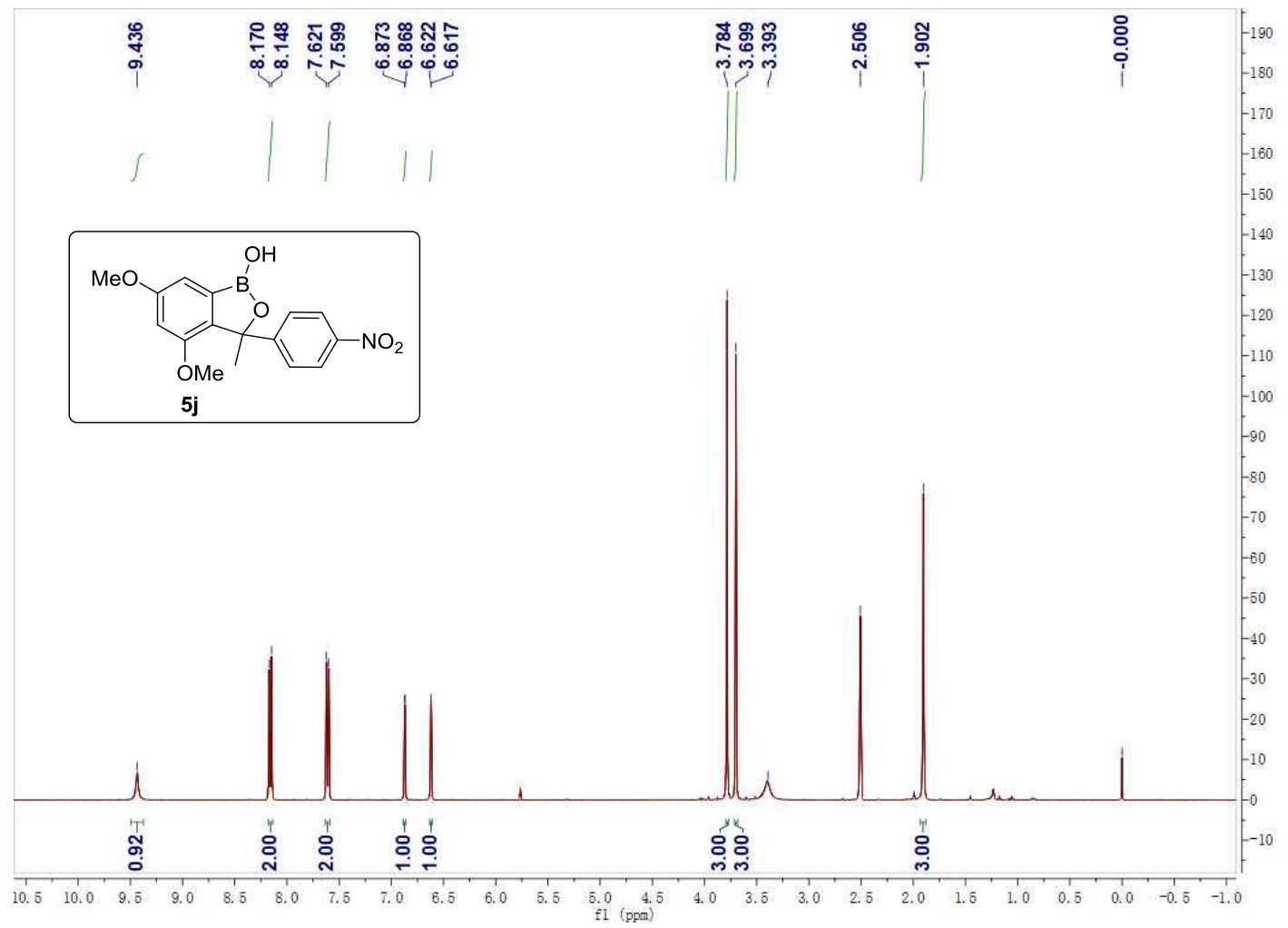

${ }^{13}$ C NMR (125 MHz, DMSO- $\left.d 6\right)$

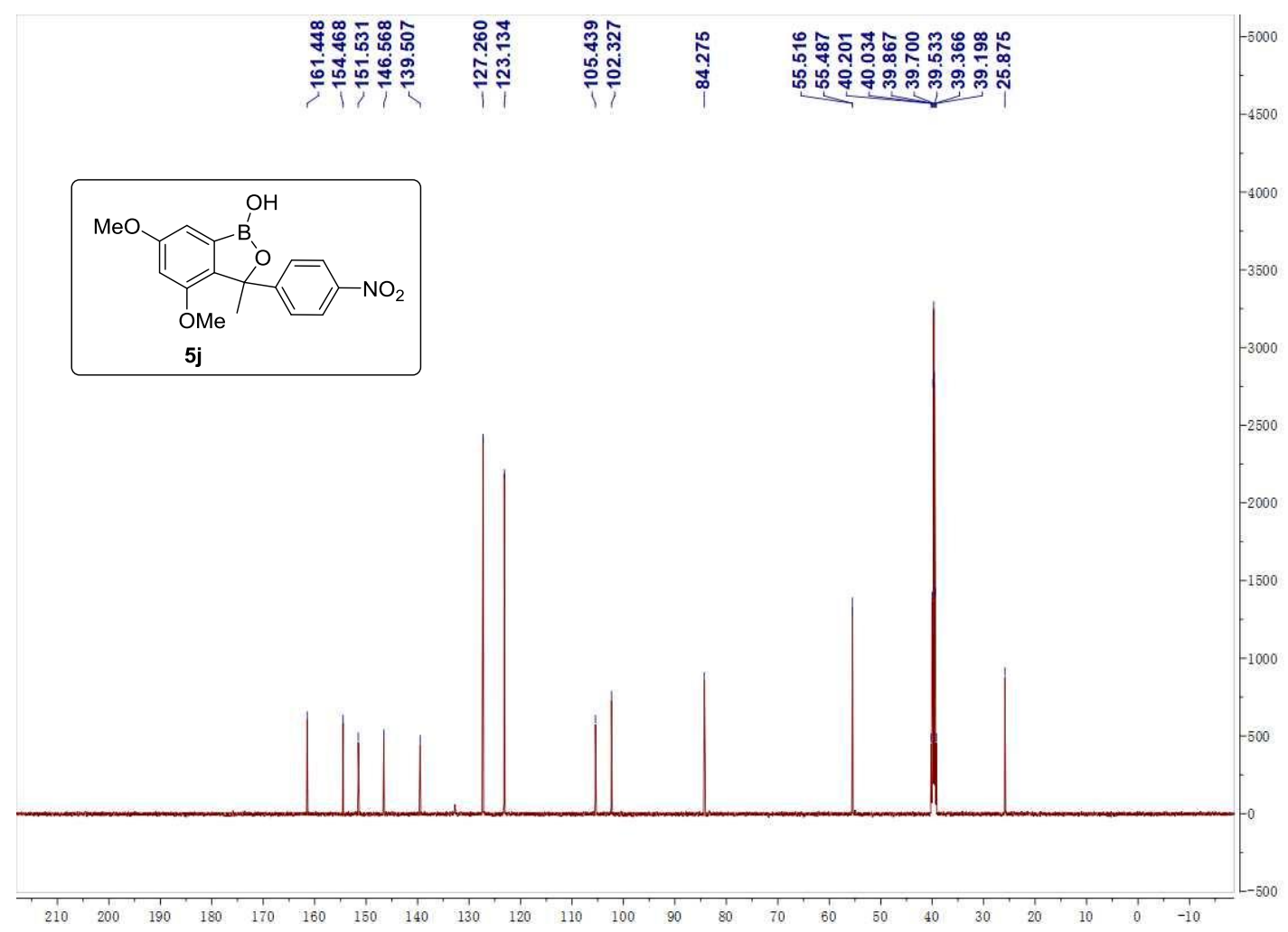


methyl 2-(1-hydroxy-4,6-dimethoxy-1,3-dihydrobenzo[c] [1,2] oxaborol-3-yl) acetate (6)

${ }^{1}$ H NMR (400 MHz, DMSO-d6)

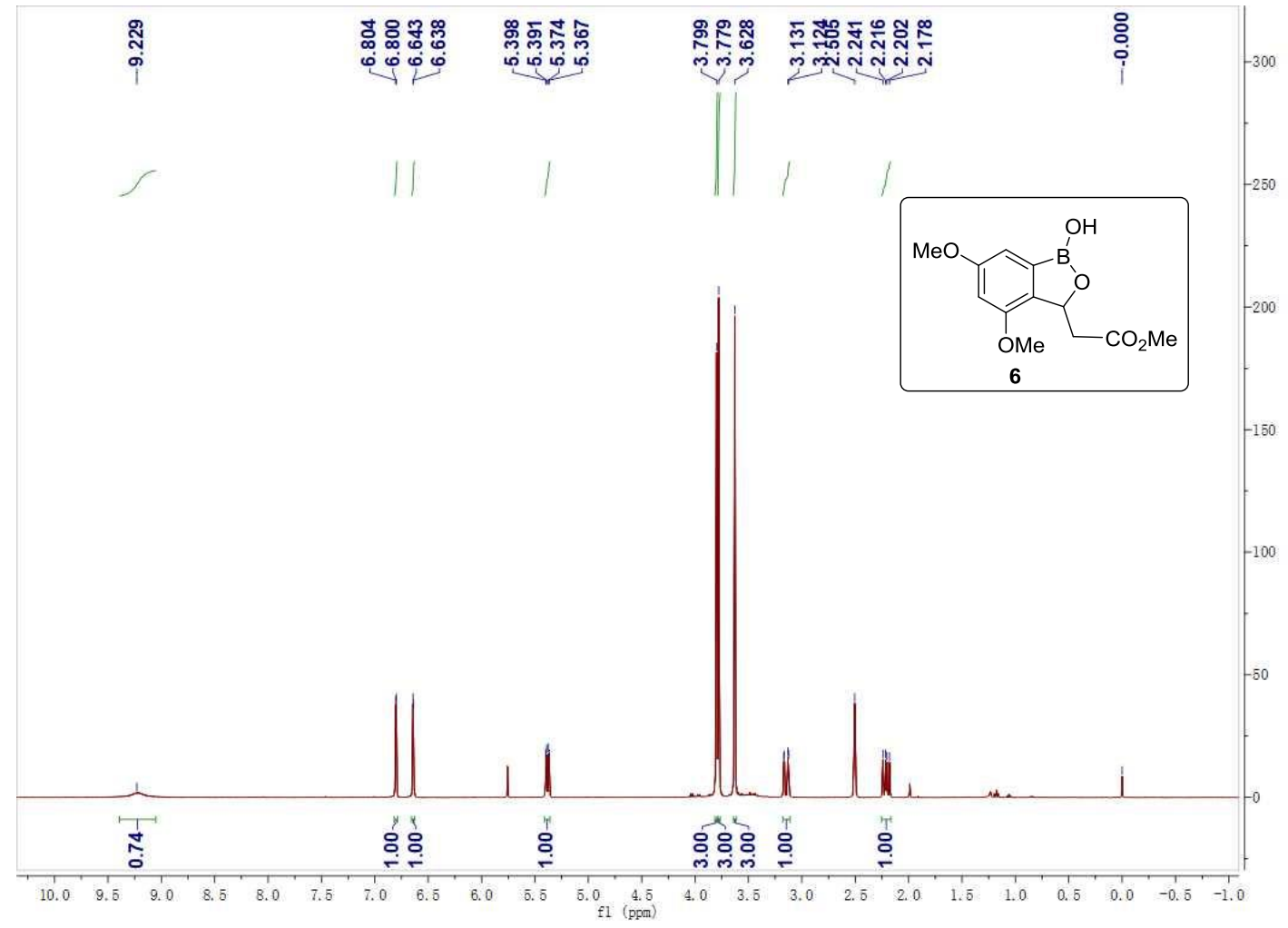

${ }^{13}$ C NMR (125 MHz, DMSO- $\left.d 6\right)$

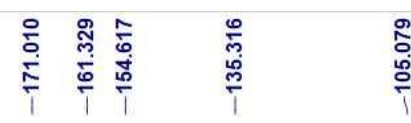

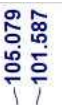

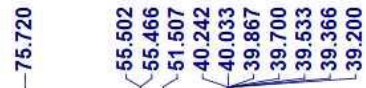
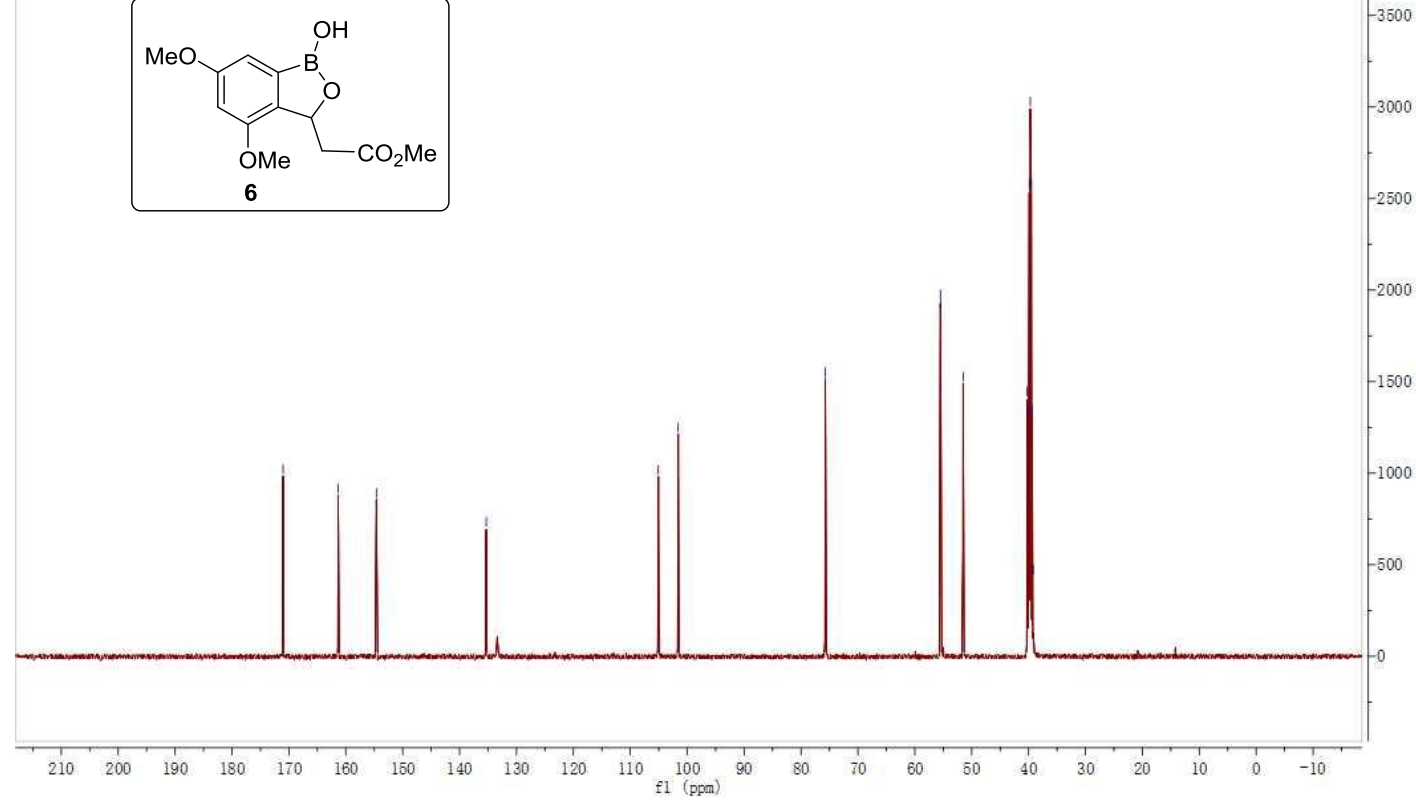
2-(1-hydroxy-4,6-dimethoxy-1,3-dihydrobenzo[c][1,2]oxaborol-3-yl)acetic acid (F)

${ }^{1}$ H NMR (500 MHz, DMSO-d6)

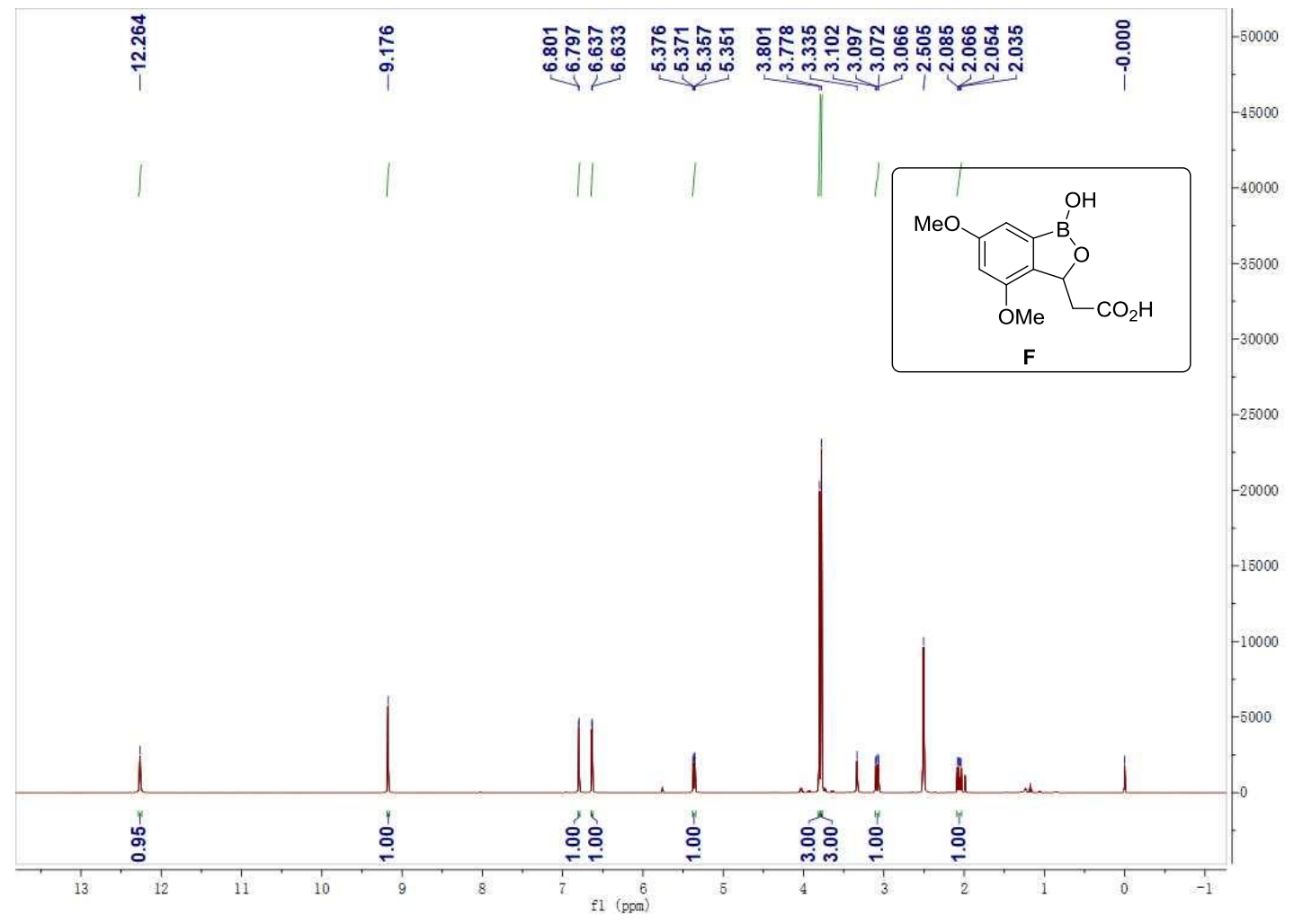

${ }^{13}$ C NMR (125 MHz, DMSO- $\left.d 6\right)$

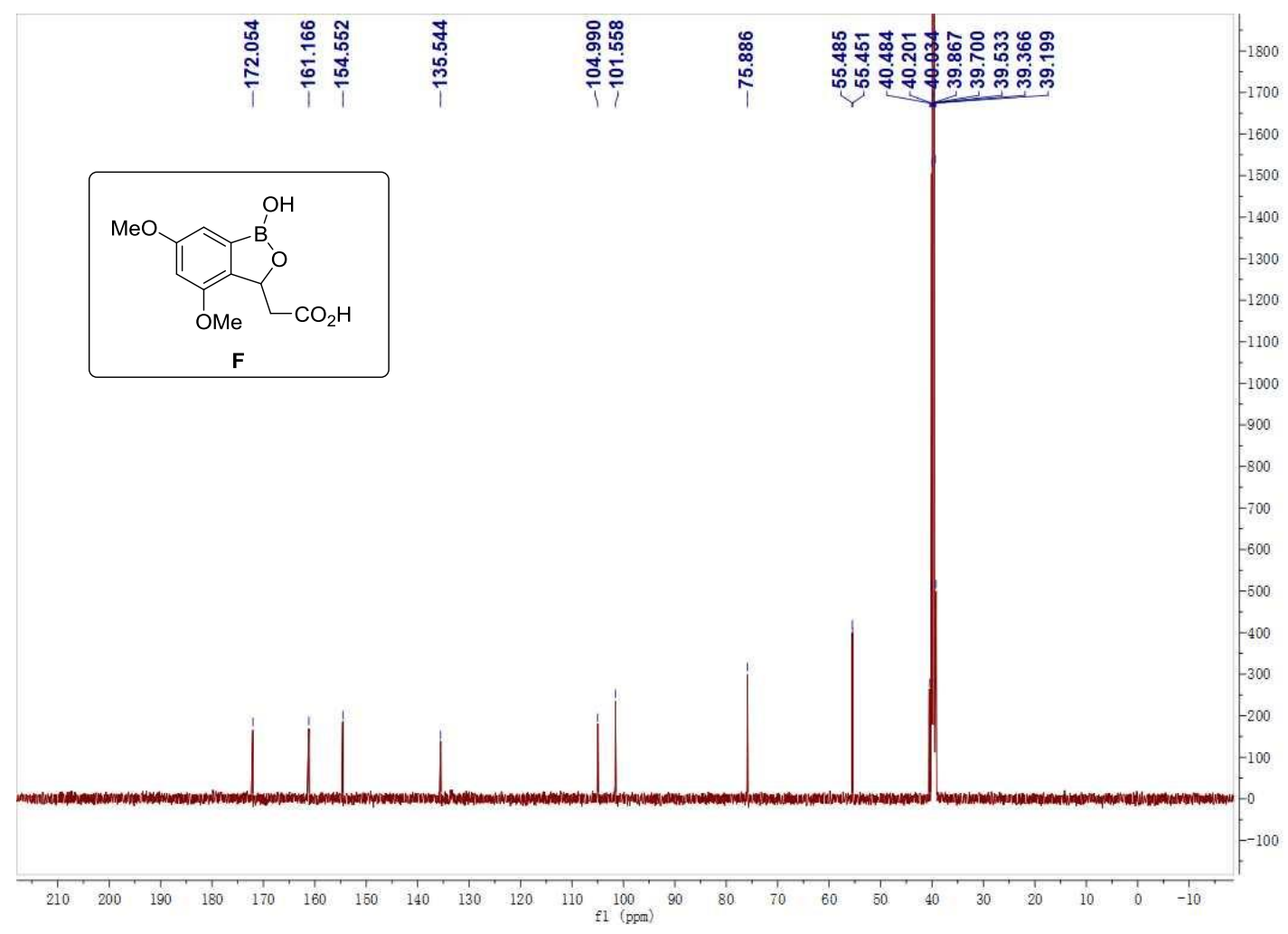

